I N T ER N ATIONAL MONETARY FUND

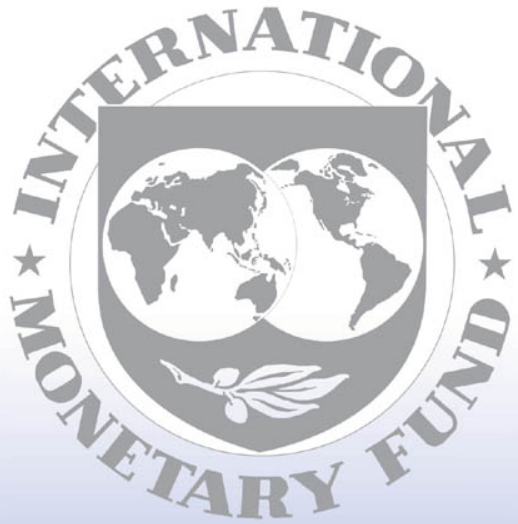

Staff

Country

Reports 
Tanzania: Seventh Review Under the Policy Support Instrument, Second Review Under the Exogenous Shocks Facility, and Request for a New Three-Year Policy Support Instrument—Staff Report; Staff Supplement; Press Release on the Executive Board Discussion; and Statement by the Executive Director for Tanzania.

In the context of the seventh review under the policy support instrument, second review under the exogenous shocks facility, and request for a new three-year policy support instrument, the following documents have been released and are included in this package:

- $\quad$ The staff report for the Seventh Review Under the Policy Support Instrument, Second Review Under the Exogenous Shocks Facility, and Request for a New Three-Year Policy Support Instrument, prepared by a staff team of the IMF, following discussions that ended on April 26, 2010 with the officials of Tanzania on economic developments and policies, and based on information available at that time. The staff report was completed on May 18, 2010. The views expressed in the staff report are those of the staff team and do not necessarily reflect the views of the Executive Board of the IMF.

- $\quad$ Supplement of May 18, 2010, updating the Joint Bank-Fund Debt Sustainability Analysis.

- $\quad$ A Press Release summarizing the views of the Executive Board as expressed during its June 4, 2010 discussion of the staff report that completed the request and/or review.

- A statement by the Executive Director for Tanzania.

The documents listed below have been separately released.

Letter of Intent sent to the IMF by the authorities of Tanzania*

Memorandum of Economic and Financial Policies by the authorities of

Tanzania*

Technical Memorandum of Understanding*

Financial System Stability Assessment—Update

*Also included in Staff Report

The policy of publication of staff reports and other documents allows for the deletion of market-sensitive information.

Copies of this report are available to the public from International Monetary Fund $\bullet$ Publication Services

$70019^{\text {th }}$ Street, N.W. • Washington, D.C. 20431

Telephone: (202) 623-7430 • Telefax: (202) 623-7201

E-mail: publications@imf.org Internet: http://www.imf.org

\section{International Monetary Fund Washington, D.C.}


INTERNATIONAL MONETARY FUND

UNITED REPUBLIC OF TANZANIA

\title{
Seventh Review Under the Policy Support Instrument, Second Review Under the Exogenous Shocks Facility, and Request for a New Three-Year Policy Support Instrument
}

\author{
Prepared by the African Department \\ (In consultation with other departments)
}

Approved by Saul Lizondo and Dominique Desruelle

May 18, 2010

- Discussions: Discussions were held in Dar es Salaam during March 2-16, and concluded in Washington during April 19-26. The team comprised David O. Robinson (head), Laure Redifer, Matthew Gaertner (all AFR), Daehaeng Kim (FAD), and Chris Papageorgiou (SPR). The team met with the Minister for Finance and Economic Affairs, Mr. Mkulo, the Governor of the Bank of Tanzania, Professor Ndulu, the Permanent Secretary of the Treasury, Mr. Khijjah, other senior officials, and representatives of the private sector, civil society and development partners. Mr. Ndyeshobola (OED) participated in the discussions.

- $\quad$ Fund relations: A PSI-supported program was approved by the Executive Board on February 16, 2007. The PSI-supported program was extended until May 29, 2010 at the time of Executive Board approval of a 12-month arrangement under the High Access component of the ESF arrangement in the amount of SDR 218.79 million (110 percent of quota) on May 29, 2009. The sixth review of the PSI-supported program and the first review of the ESF arrangement were approved on November 23, 2009, with cumulative ESF disbursements of SDR 198.9 million.

- $\quad$ Reviews: Staff recommends completion of the seventh review under the PSI and the second review under the ESF arrangement - all end-December performance / assessment criteria were met, and reasonable progress was achieved in implementing structural reforms. The third and final disbursement of SDR 19.89 million would become available upon completion of the review of the ESF arrangement.

- $\quad$ Request for new PSI. The authorities have requested a new 3-year PSI-supported program to support their efforts to accelerate pro-poor growth to ensure a sustainable reduction in poverty - a new 5-year poverty reduction and growth strategy is expected to be approved in June. Staff support this request based on understandings reached on a medium-term macroeconomic policy framework and specific policies and program targets for FY 2010/11; Tanzania's comfortable level of reserves and medium-term developmental needs continue to make the PSI an appropriate mode of engagement. 
Executive Summary ..................................................................................................... 4

I. Strong Macroeconomic Gains, Slower Progress Toward the MDGs .................................. 5

II. Emerging from the Global Financial Crisis and Local Shocks ........................................ 8

A. Program performance........................................................................................... 13

III. A Renewed Focus on Pro-Poor Growth..............................................................

A. Macroeconomic Framework ................................................................................... 16

B. Creating Fiscal Space and its Effective Usage........................................................ 18

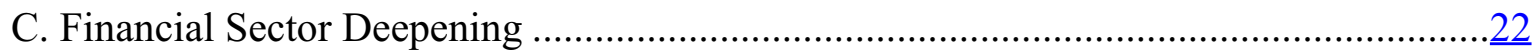

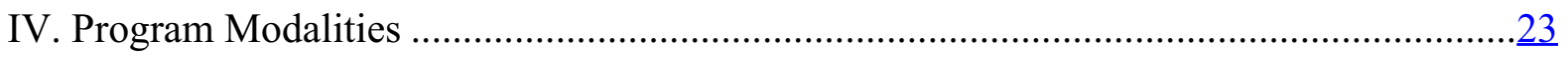

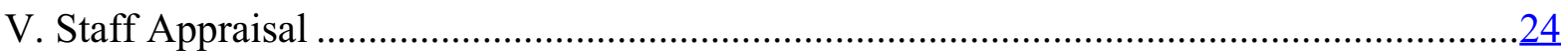

Tables

1. Selected Economic and Financial Indicators, 2007/08-2012/13 ....................................26

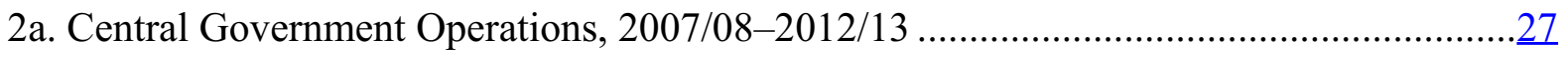

2b. Central Government Operations, 2007/08-2012/13 ….............................................

3. Monetary Accounts, 2007/08-2010/11 .......................................................................29

4. Summary Accounts of the Bank of Tanzania, 2009/10-2010/11 ....................................30

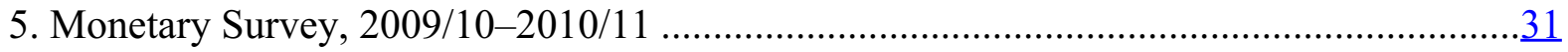

6. Balance of Payments, 2007/08-2012/13 .....................................................................

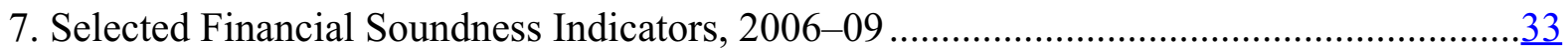

Figures

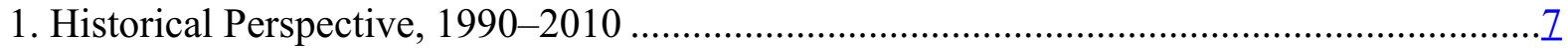

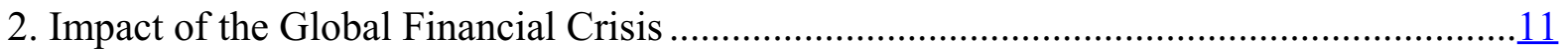

3. Monetary and Exchange Rate Developments .........................................................12

Boxes

1. Performance Under the 2007-10 PSI-Supported Program ...............................................

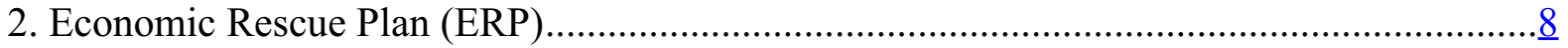

3. New Poverty Reduction and Growth Strategy (MKUKUTA II) …................................15

4. Tax Revenue Improvements - Policy Options ......................................................... 20 
Appendix

I. Letter of Intent..... .$\underline{34}$

Attachment I. Memorandum of Economic and Financial Policies .................................. $\underline{36}$

Table 1. Quantitative Assessment/Performance Criteria and Indicative Targets Under the Policy Support Instrument and the Exogenous Shocks Facility, September 2009-March 2010

Table 2. Quantitative Assessment Criteria and Indicative Targets Under the Policy Support Instrument, June 2010-June 2011

Table 3. Proposed Structural Benchmarks During First Year of New PSI, 2010/11 ....... $\underline{48}$

Attachment II. Technical Memorandum of Understanding on Selected Concepts and

Definitions Used in the Monitoring of the PSI-Supported Program

Table 1. Summary of Reporting Requirements 


\section{Executive Summary}

- $\quad$ Performance under the PSI-supported program/ESF arrangement remains strong — all quantitative performance/assessment criteria for end-December 2009 were observed. Progress on structural reforms has been mixed, but the two measures that were not completed in the anticipated time frame are expected to be achieved in June. Staff recommends completion of the $7^{\text {th }}$ review under the PSI and $2^{\text {nd }}$ review of the ESF.

- The economy is starting to emerge from a slowdown associated with the global crisis with growth estimated to have been 5.5 percent in 2009 and projected to reach 6.2 percent in 2010. Monetary and fiscal easing, together with an economic rescue plan, has helped cushion the economy. Inflation was persistently high in 2009, but has recently come down to single digits with the abatement of regional food supply shocks. Monetary and fiscal stimulus will be withdrawn gradually in order to protect the still nascent recovery.

- $\quad$ The authorities are preparing a new poverty reduction and growth strategy (MKUKUTA II) covering 2010-15 that is expected to be approved in June. Key objectives are: to accelerate growth and the reduction of income poverty; to improve quality of life and social well-being; and to strengthen governance and accountability. The strategy is based on measures to enhance agricultural productivity - to boost rural incomes and improve food security - a scaling up of infrastructure and improvements in public financial management.

- Implementation of MKUKUTA II will require the creation and effective use of additional fiscal space. There are options for rationalizing spending as well as to enhance revenues which, despite significant improvements in recent years, remain well below potential. Grants and concessional borrowing will remain the main source of financing for investment spending, but are likely to be insufficient to support a large scaling up of infrastructure investment. The authorities estimate that nonconcessional borrowing of $\$ 1.5$ billion would be required over the three years of the PSI (1.8 percent of GDP per year), together with increased private sector participation through public private partnerships. The updated DSA prepared jointly by the staffs of the World Bank and IMF continues to point to a low risk of debt distress.

- The authorities have requested a new 3-year PSI to support the implementation of MKUKUTA II. The new PSI will focus on the macroeconomic framework to support accelerated growth, creating additional fiscal space while enhancing the return on public spending, and containing vulnerabilities. Staff supports the authorities' request. 


\section{Strong MACroeconomic Gains, SLOWER PROGRess TOWARD THE MDGs}

1. Macroeconomic outcomes have strengthened dramatically over the last decade (Figure 1). Growth has accelerated from about 3.5 percent in the $1990 \mathrm{~s}$ - barely above the rate of population growth - to an average of over 7 percent, inflation has remained under control, international reserves have increased from less than $\$ 0.5$ billion to $\$ 3.5$ billion, and there have been no major banking or foreign exchange crises. Fiscal space was created by a combination of debt relief and determined efforts to improve tax administration, the latter resulting in a significant increase in domestic revenues. Fiscal space has permitted a significant expansion in public spending, while preserving macroeconomic stability, with an increased share of spending being directed to priority sectors as defined under the national poverty reduction and growth strategy (MKUKUTA). Macroeconomic policies during this period were supported by close engagement with the Fund - most recently a PSI-supported program approved in February 2007, following earlier PRGF and ESAF arrangements (Box 1).

2. Social outcomes improved during this period, and Tanzania is on track to achieve about half of the MDGs. Increased public spending has resulted in substantial improvements in access to basic health and education services: for example, primary school enrolment rates have risen dramatically and under-five and infant mortality rates have declined sharply. However, there has been limited progress in reducing income poverty and in addressing indicators of malnutrition, and maternal mortality rates have risen.

\section{Program review and new program discussions took place against a backdrop} that included the preparation of a new 5-year poverty reduction and growth strategy (MKUKUTA II) and forthcoming Presidential and Parliamentary elections. Drawing on a set of evaluation studies of developments under the existing MKUKUTA, a draft new strategy was circulated to stakeholders in early April, with national consultations expected in May/June prior to formal adoption of the new strategy by end-June. Elections are scheduled for October 31, 2010. The CCM party, which has been in power continuously since independence in 1962, is well ahead in the polls. 


\section{Box 1. Performance under the 2007-10 PSI-Supported Program}

Objectives: The PSI-supported program was approved in February 2007 in order to support the authorities' efforts to achieve sustainable broad-based high growth and poverty reduction, while maintaining macroeconomic stability. Consistent with this overarching objective, there were three main pillars: fiscal reform to enhance domestic revenue and increase the efficiency of spending; acceleration of financial sector reform; and improvements to the business environment to stimulate private sector investment.

Macroeconomic performance: Real GDP growth remained close to 7 percent in the first two years of the program and proved relatively resilient during the financial crisis. Prudent fiscal policy provided the necessary space to implement a countercyclical fiscal response to the crisis that helped mitigate weaker external demand and a sharp slowdown in credit growth. Inflation rose into double digits during 2008-09, mostly reflecting supply-side shocks that pushed up food prices, but fell sharply in the first half of 2010 as these effects subsided. The reserve money target continued to provide an appropriate anchor for monetary policy, while significant improvements were made in the operational framework, including systemic liquidity management and foreign exchange operations.

Public resource mobilization and efficiency of spending: Revenue collection rose significantly in the first year of the program, supporting increases in priority expenditures on health and education, but fell short of the targeted improvement in 2009/10 largely due to the impact of the financial crisis. Considerable progress has been made in addressing governance issues, but progress on public financial management has been mixed and will need to be strengthened in order to increase space for infrastructure investment without comprising key social expenditures.

Financial sector: Financial intermediation has increased significantly in recent years, with the ratio of private credit to GDP rising from 12.7 percent at the end 2006 to 16 percent at end-2010 due to rapid credit growth, but weak creditor rights continue to constrain lending and access to finance remains below the rest of the region.

Business environment: Infrastructure gaps constitute a key constraint on long-run growth, and will need to be accompanied by greater efforts to strengthen the investment climate, where progress has been limited.

Access to Fund financing: In early 2009, in the face of a significant deterioration in balance of payments prospects, the authorities requested a one-year ESF arrangement in the amount of 110 percent of quota. The presence of the PSI and the authorities' strong track record of implementation enabled rapid agreement on the ESF arrangement. The authorities consider the ESF arrangement to have played a valuable role in maintaining confidence and limiting potential contagion. 
Figure 1: Historical Perspective, 1990-2010

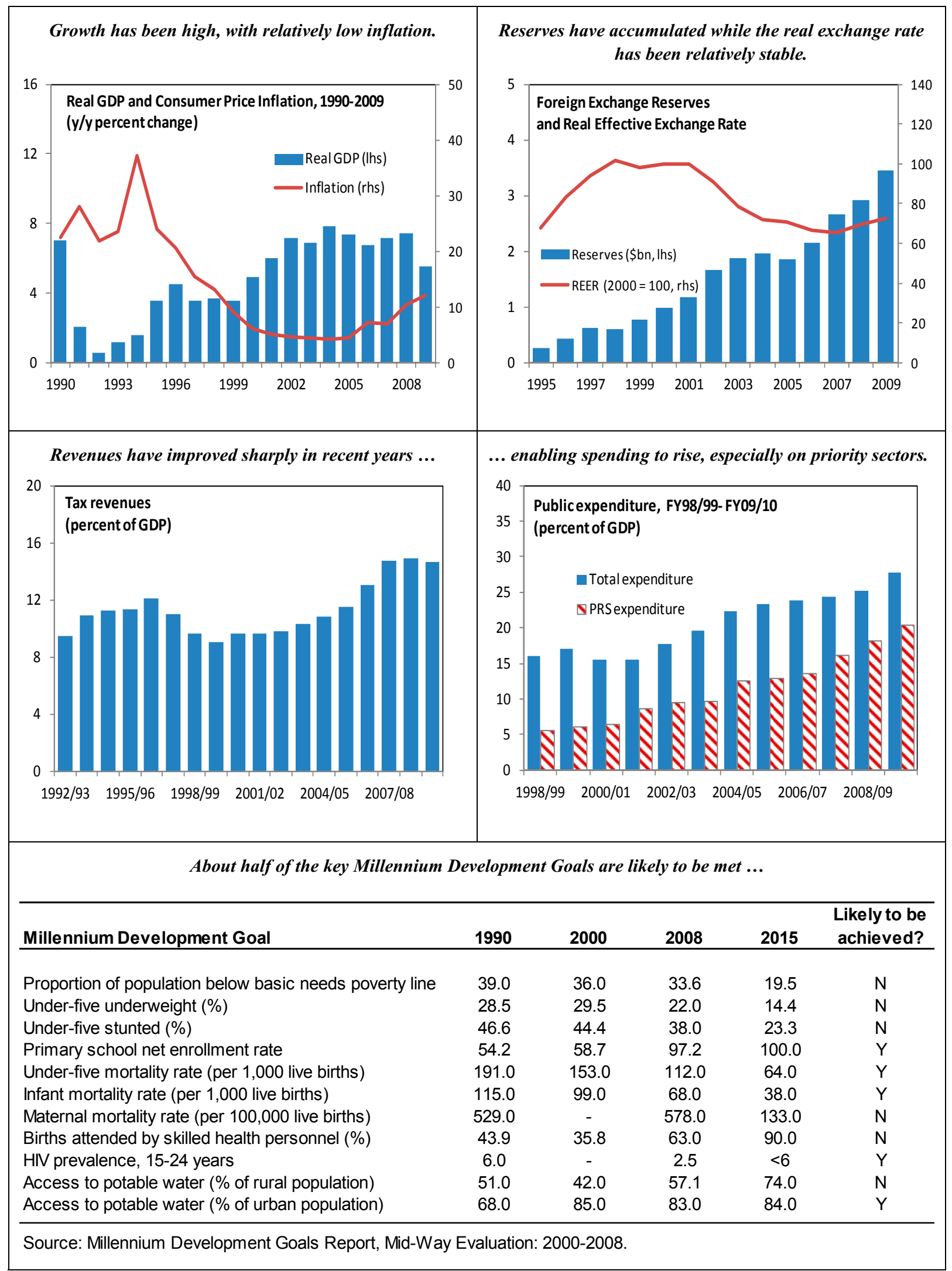




\section{Emerging from the Global Financial Crisis ANd Local SHOCKS}

4. Monetary and fiscal easing, together with specific interventions under the Economic Rescue Plan, helped contain the immediate impact of the global crisis, but private sector recovery remains nascent (Box 2 and Figure 2). Preliminary data suggest stronger than anticipated growth in traditional exports, tourism, construction, and mining, despite a series of shocks-drought in the North, rolling electricity outages, and floods disrupting the main north-south rail route. However, non-oil imports are largely flat, while private sector credit growth has fallen sharply. Tax collections have improved in the last several months, but still remain well below target.

\section{Box 2: Tanzania's Economic Rescue Plan (ERP)}

The authorities implemented a package of fiscal measures in FY09/10 to cushion the impact of the global financial crisis, supported by monetary policy easing. The key objectives were to protect: employment and incomes; food security; infrastructure investment; and social services programs. The Fund welcomed the ERP as an appropriate response to the crisis, and provided balance of payments support to bolster reserves.

The ERP includes on-budget measures to support a projected fiscal easing of over 2 percent of GDP. Specific measures include: a reduction in the VAT rate from 20 to 18 percent; expanded government loan guarantees, including partial government guarantees on troubled loans restructured by commercial banks; temporary price supports for cotton and expanded agricultural input subsidies; expanded investments in roads and energy; and temporary exemptions from royalties for tanzanite and diamond miners. The ERP included financing measures, such as domestic market borrowing and exceptional credit from the Bank of Tanzania, and was supported by additional financing from donors.

The expenditure measures in the ERP appear to have been helpful in mitigating the impact of the global crisis by providing support to affected sectors, particularly in rural areas where poverty is concentrated. However, an in-depth analysis of the program's effectiveness would be premature, especially for agriculture. By the end of the fiscal year on June 30, most, although not all, additional spending is expected to have been carried out. The VAT rate reduction - which brought the rate in line with most other countries in the East African Community (EAC) region - is expected to be permanent, but has not had a strong adverse impact on revenue collection thus far. Given the lack of a formalized social safety net, targeted discretionary spending measures were the best means for protecting affected and vulnerable sectors, while providing some boost to aggregate demand, although multiplier effects for growth were not necessarily a first order objective.

5. Inflation, persistently high during 2009 due to large increases in food prices, has declined to single digit levels. Headline CPI for March 2010 (9 percent y/y) reflects a sharp 
deceleration in food price inflation (9.7 percent, compared with an average of 17 percent during the second half of 2009) as the impact of regional drought dissipated. Higher energy prices, reflecting the pass-through of higher global prices, have added to inflationary pressures.

6. Revenues continue to underperform relative to the budget, resulting in a modest scaling back in expenditure to limit deterioration of the fiscal deficit. Cumulative revenues for the first three quarters of the fiscal year were about 91 percent of the budget target - the recent growth acceleration was led by lightlytaxed sectors - while some policy measures underlying the revenue estimates, e.g. the removal of fuel levy

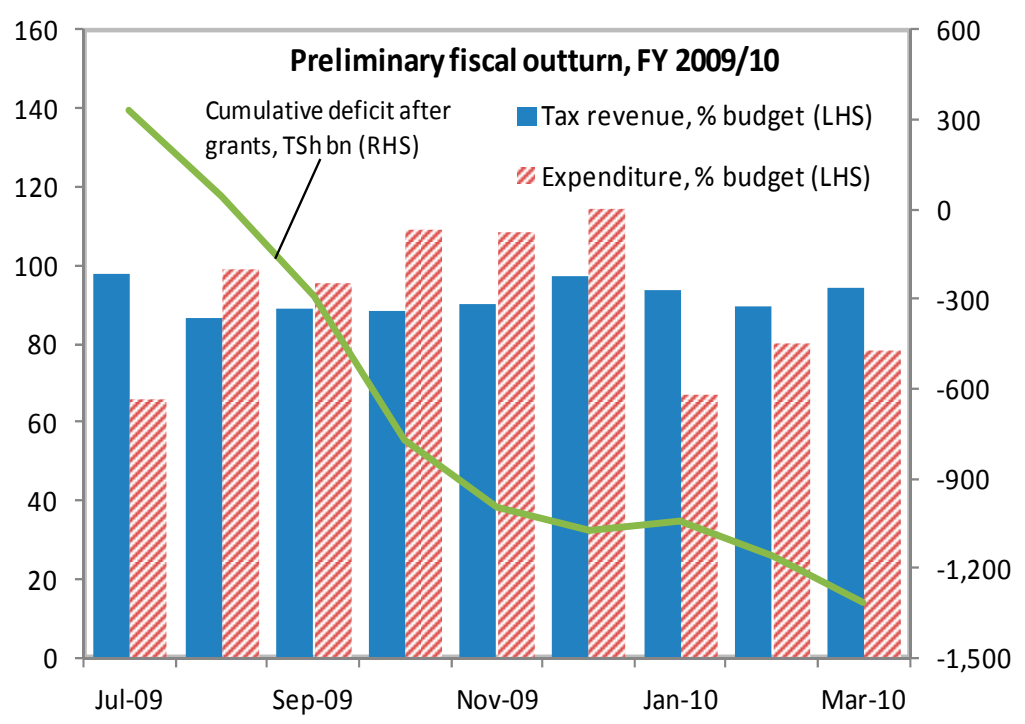
exemptions, have yet to be implemented. Despite a reduction in the VAT rate from 20 percent to 18 percent, VAT collection has been relatively strong. Spending - including domestically financed development spending - has been implemented broadly in line with the budget for most of the year, as the shortfall in revenues was compensated by a front-loading of financing from development partners. In recent months the pace of spending has slowed, resulting in cumulative budget execution of 91.4 percent through the first three quarters of FY 2009/10.

7. Monetary policy has produced a low interest rate environment, but private sector credit growth has slowed sharply (Figure 3). In the face of weak money demand, the Bank of Tanzania (BoT) has sought to sterilize government spending associated with aid inflows to contain a liquidity build up in the banking system. Interest rates remain lowwith deposit rates negative in

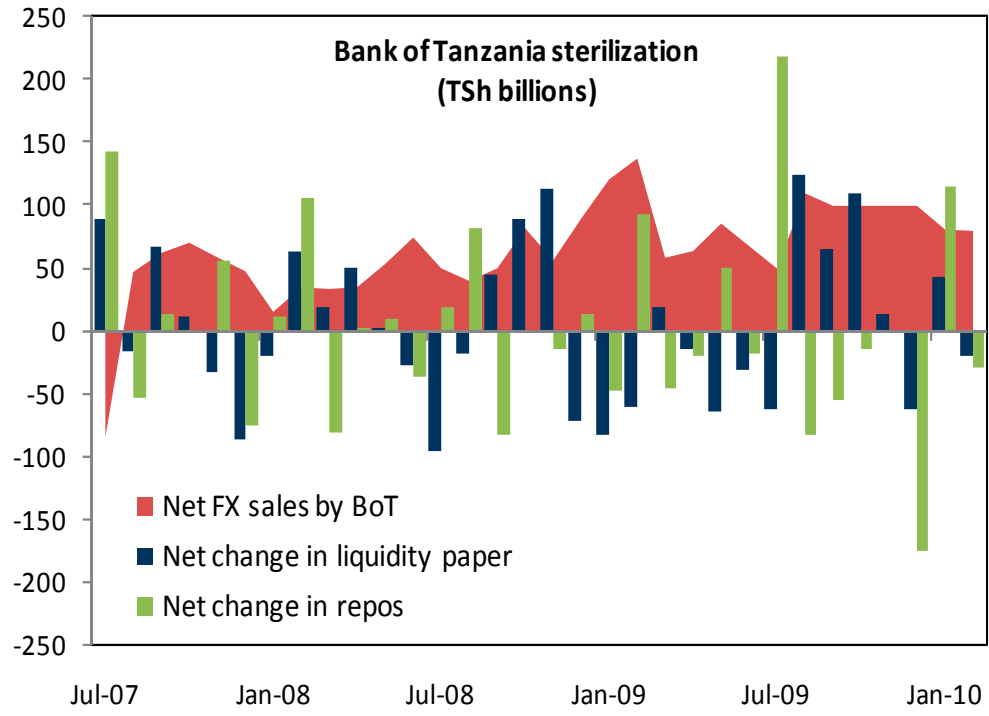
real terms - but private sector credit growth continued to slow sharply in the second half of 
2009 as commercial banks adopted more cautious lending strategies, reflecting concerns about the impact of the global financial crisis on key sectors.

\section{The exchange rate has depreciated modestly in nominal terms, with less} volatility than seen in neighboring countries. Timely disbursements of budget support by development partners, a stronger than anticipated current account, together with the ESF disbursements and the SDR allocation, provided a comfortable reserve cushion that limited speculative pressures in the foreign exchange market. The BoT has been able to maintain a steady pace of foreign exchange sales as one component of sterilization operations. The real effective exchange rate remained almost unchanged during 2009.

\section{Financial soundness indicators of the banking system appear sound, though} profitability has fallen. ${ }^{1}$ The system remains well capitalized, with large domesticallyowned banks increasing capital during the year. NPLs increased during the height of the crisis, but appear to have peaked in mid-2009. Return on assets has fallen with lower interest rates, but remains high by international standards.

\footnotetext{
${ }^{1}$ Interpretation of recent trends in FSIs is complicated by the unclear impacts of measures in the economic recovery plan-limited debt write-offs and partial government guarantees for restructuring of certain classes of loans. In addition, gaps in prudential data collection and analysis hinder a comprehensive assessment of systemic and individual risks in the financial sector.
} 
Figure 2. Impact of the Global Financial Crisis

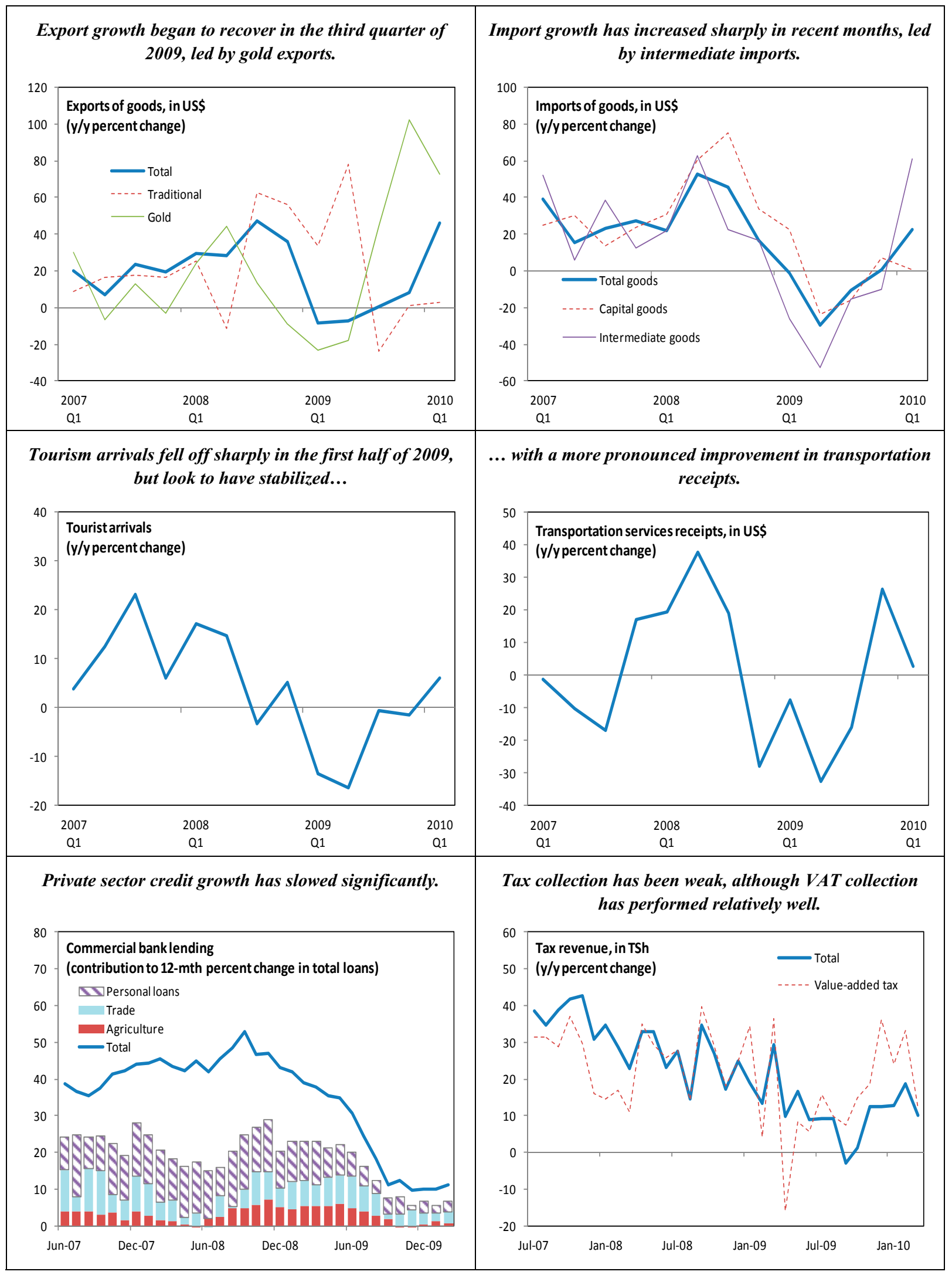


Figure 3. Tanzania. Monetary and Exchange Rate Developments

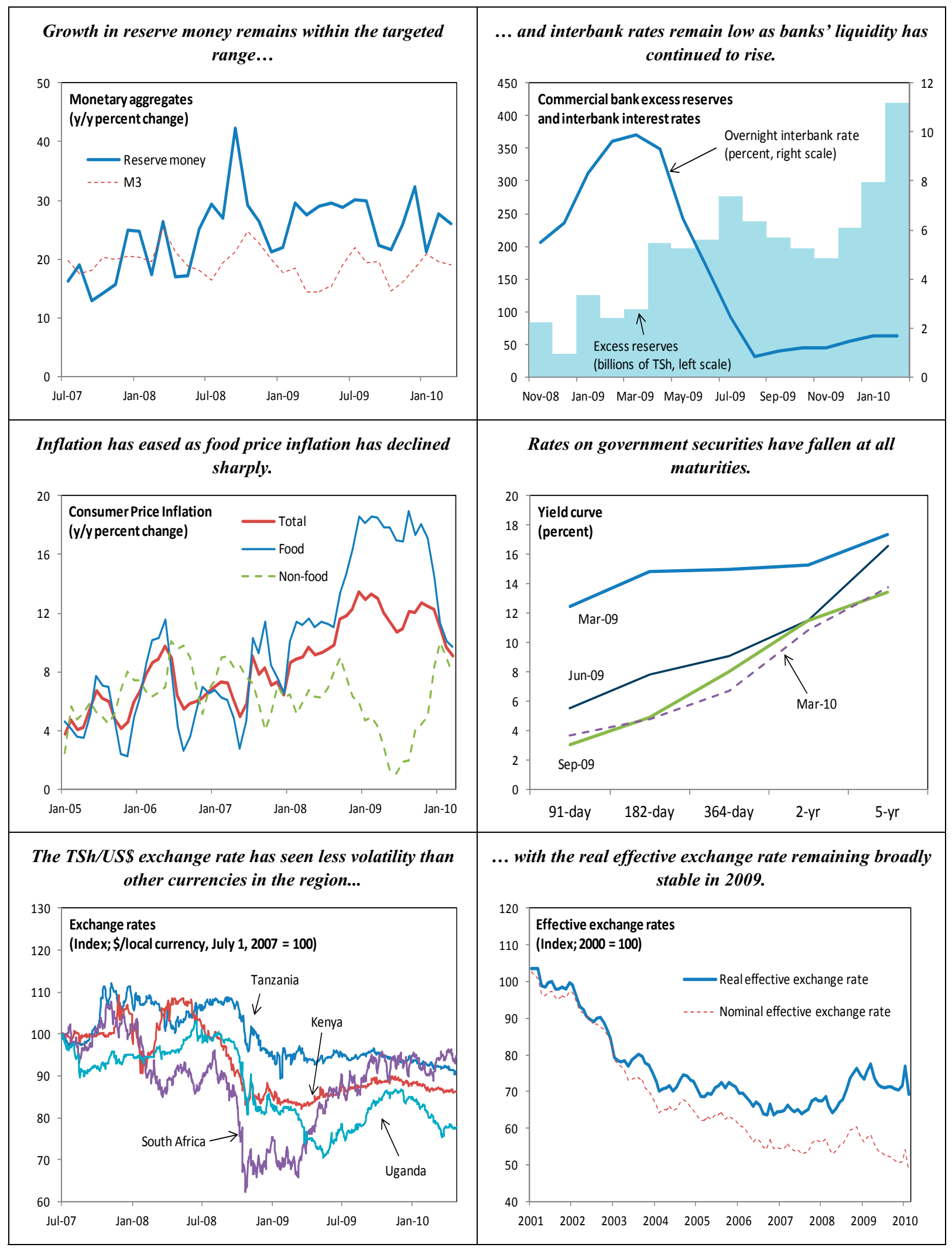




\section{A. Program performance}

10. All end-December quantitative performance/assessment criteria were met, and preliminary data suggest that end-March indicative targets were also observed. NIR remained comfortably above its floor, reflecting stronger than anticipated balance of payments inflows. Reserve money was at its target midpoint in December, but was held below the target range in March as money demand weakened and the BoT sought to limit build up in excess liquidity in the banking system.

11. Implementation of the structural agenda has been mixed (Text Table 1). An updated financial stability report is under preparation and is now expected to be presented to the BoT Board in June 2010, rather than December 2009. Issuance of investment guidelines for pension funds has been further delayed, awaiting appointment of a social security regulator. Initial efforts to recruit a social security regulator were unsuccessful in identifying a qualified candidate - a second recruitment effort is underway and is expected to be concluded shortly, enabling the issuance of guidelines by end-June. Other structural measures, relating to a strategy for refocusing the BoT on core activities, improving cash flow forecasting and developing a functional classification of the budget, have been met. 
Text Table 1. Tanzania: Structural Benchmarks for 2009/10

\begin{tabular}{lll}
\hline \multicolumn{1}{c}{ Measure } & Target Date of & Status \\
\hline Financial Sector
\end{tabular}

Adopt a strategy for refocusing the BoT on its core activities.

Issue investment guidelines for pension funds prepared by the BoT.

Prepare an updated financial stability report for the BOT Board, including assessments of risk-based prudential supervision.

\section{Fiscal}

Cash Management Unit (CMU) in the Accountant General's Department to produce Government's three month rolling cash-flow forecast.

Prepare a functional classification of expenditures consistent with the IMF's Government Financial Statistics Manual 2001 for the budget for 2010/11.
End-May 2009

Met with delay. Time-bound action plan underway, and BoT has already implemented the key elements.

End-June 2009

Ongoing. Is suance awaits appointment of a head of the Social Security Regulatory Agency. A second recruitment round has been undertaken to identify a suitable candidate. Hiring of the regulator and issuance of guidelines are expected by endJune.

End-December 2009

Ongoing. Draft report is finished and will be presented to the BoT Board in June.

Met

End-April 2010

Met. Functional classification has been prepared, and preparations are on track to report spending on a functional basis starting in FY10/11. 


\section{A Renewed Focus on Pro-Poor Growth}

\section{The new MKUKUTA will provide an opportunity to reorient the development} strategy to accelerate progress in reducing income poverty and enhancing living standards. There is a broad consensus that implementation of the first MKUKUTA (covering 2005-10) was successful in making improvements in key social areas - notably access to health and education - but was less successful in enhancing growth that would provide the basis for sustainable improvements in income opportunities, especially in rural areas (75 percent of the population resides in rural areas which also have a higher incidence of poverty). Thus, MKUKUTA II (covering 2010-15) is anticipated to place greater emphasis on enhancing pro-poor growth, while seeking to preserve gains in social sectors (Box 3).

\section{Box 3: Tanzania's New Poverty Reduction and Growth Strategy (MKUKUTA II)}

A draft was circulated to all stakeholders in early April to form the basis for national consultations over the coming months, with formal adoption by Cabinet of a new strategy by end-June. The draft MKUKUTA II maintains three main objectives: growth and reduction of income poverty; improvement of quality of life and social well-being, and governance and accountability. Drawing on an extensive review of the existing MKUKUTA, the new strategy seeks to sustain progress achieved in social sectors while reorienting the emphasis of new spending toward promoting employment-enhancing growth, particularly through:

- Broad-ranging spending and other measures in agriculture to improve food security, boost agricultural productivity, and expand rural incomes, consistent with the Kilimo Kwanza (Agriculture First) policy;

- Interventions in other sectors identified as potential drivers of growth and employment (fishing, manufacturing, mining, and tourism);

- $\quad$ Scaled up infrastructure spending, especially in transportation, power generation, and irrigation, to stimulate growth sectors both in Tanzania and in the region; and

- $\quad$ Strengthening public sector financial management and the business environment, to ensure spending efficiency and maximize the return to MKUKUTA II interventions.

13. The new PSI will support implementation of MKUKUTA II. The new

MKUKUTA will only be in place at the time of the first review of the PSI; therefore structural benchmarks were agreed for the first program year, but medium-term structural reforms will be established once MKUKUTA II is adopted. However, it was agreed that the focus of the new PSI would build on achievements of the current PSI and generally protect macroeconomic stability and contain potential vulnerabilities. Key elements are: 
- The design of a macroeconomic framework to support accelerated growth;

- Creation of additional fiscal space to provide room for key interventions - such as the envisaged scaling-up of infrastructure investment - coupled with steps to enhance the return on public spending and limit risks to debt sustainability; and

- Deepening financial sector markets to support growth while ensuring appropriate supervision of vulnerabilities.

\section{A. Macroeconomic Framework}

\section{Remainder of FY2009/10 and FY 2010/11}

14. The economy is emerging from the slowdown induced by the global financial crisis. The authorities noted that the specific interventions contained in the economic rescue plan, combined with the fiscal stimulus had helped contain the impact of the global crisis, while ESF disbursements and the SDR allocation had had important signaling effects that helped limit pressures in the foreign exchange market.

15. Growth is expected to accelerate modestly in 2010-11, accompanied by a further decline in inflation. Real GDP growth is projected to rise to 6.2 percent in 2010 and to 6.7 percent in 2011, reflecting a continuation of the economic rebound in the second half of 2009 and the anticipated pick up in global and sub-Saharan African growth. Inflation is expected to decline to 5.0 percent by end-June 2011 as food price pressures abate in line with supply-side improvements.

\section{Fiscal policy will seek to balance a gradual reining in of the stimulus while} reorienting spending in line with MKUKUTA II objectives. Given the still nascent nature of private sector growth, the authorities intend to rein in public spending gradually. For FY10/11, the deficit before grants will be reduced to 10.8 percent of GDP from 12 percent currently projected for FY 09/10, consistent with available financing. Revenues are expected to increase by around 0.4 percent of GDP, through administrative improvements and tightening mining exemptions. On the expenditure side, the composition of spending will be reoriented to increase investment spending to meet infrastructure needs while maintaining priority social spending. This would partly offset by a 1.9 percent of GDP reduction in goods and services spending through the expiration of time-bound elements of the economic rescue plan ( 0.5 percent of GDP) and containing other non-priority goods and services expenditures (1.4 percent of GDP), including non-wage payments, travel expenditures, and equipment acquisition. This would also allow a modest ( 0.4 percent of GDP) increase in the wage bill, in order to alleviate human resource constraints in the provision of key social services.

17. Monetary policy will continue to provide a supportive environment for recovery, while carefully monitoring inflationary pressures. The FY 2010/11 financial program includes an increase in average reserve money of 18.5 percent, reflecting an increase in money demand as private sector recovery accelerates and efforts underway to expand access to formal financial services, with a modest reduction of excess reserves in the banking 
system. Private sector credit is expected to increase by 20 percent, still below the very high rates seen in the pre-crisis period. The BoT will monitor developments closely to ensure that the reserve money target is consistent with emerging demand. The BoT plans to maintain regular foreign exchange sales to meet systemic liquidity management objectives. The authorities are updating the CPI basket, and working with EAC counterparts to develop a common core inflation index, which would serve as a better guide to the monetary policy stance.

\section{Medium-term}

18. Developments over the medium-term are likely to reflect a continuation of the broad policy strategy employed in FY2010/11. The fiscal deficit will remain higher than in recent past to accommodate higher infrastructure spending; the composition of spending will be reoriented to growth-enhancing expenditures; and monetary policy will seek to support an acceleration in growth while maintaining a low inflation environment without excessive volatility in the exchange rate. The base scenario incorporates a gradual acceleration of growth to 7.5 percent by $2012 / 13$, in part reflecting the initial returns from the increased investment, while inflation would stabilize at around 5 percent. Key uncertainties for the growth outcome include the rate of return on public investment and the responsiveness of private sector activity, while the inflation path would be vulnerable to external shocks - both to food supply and energy prices_ — as well as, potentially, demand pressures. ${ }^{2}$

Text Table 2. Medium-term Macroeconomic Scenario

\begin{tabular}{lrrrr} 
& $2009 / 10$ & $2010 / 11$ & $2011 / 12$ & $2012 / 13$ \\
\hline Fiscal deficit (exc. grants) / GDP & 12.0 & 10.8 & 10.0 & 8.9 \\
Fiscal deficit (inc. grants) / GDP & 5.7 & 5.9 & 5.4 & 4.6 \\
Consumer price inflation (avg) & 10.6 & 5.8 & 5.0 & 5.0 \\
Gross official reserves (months of imports) $^{\text {Real GDP growth }}{ }^{1}$ & 5.2 & 5.0 & 5.0 & 5.0 \\
Current account deficit (exc. grants) / GDP $_{\text {Public debt/GDP }}^{5.5}$ & 6.2 & 6.7 & 7.5 \\
\hline
\end{tabular}

${ }^{1}$ Data are on a calendar year basis. For example, 2009/10 data are for calendar year 2009.

19. A formal social protection framework is an important component that can help ensure broader participation in income gains. The global crisis combined with the regional drought exposed key holes in the social protection framework with interventions in the ERP largely untargeted, with heavy reliance on donor and civil society instigated

\footnotetext{
2 The analysis in Scaling-Up Aid in Tanzania (2010, forthcoming) by Matthew Gaertner and Laure Redifer suggests considerable room to expand spending without creating undue strains on factor or product markets.
} 
projects, and ad hoc bans on the export of agricultural products due to food security concerns. A social protection framework is expected to be approved in June, which will form the basis for developing costed options for supporting the most vulnerable groups.

\section{B. Creating Fiscal Space and its Effective Usage}

\section{Policy interventions envisaged under MKUKUTA II will require additional and} more effective use of fiscal space. The authorities noted that addressing the infrastructure weaknesses is likely to require substantial investments that go beyond the government's own resources and the likely availability of concessional financing. Thus various options for raising additional resources - both quasi-concessional and nonconcessional borrowing as well as greater private sector participation - are under consideration. Staff agreed on the need to address infrastructure needs (see Text Table 3), but stressed that efforts should also be made to create additional resources within the budget - through rationalization of expenditures and enhanced revenue efforts - to complement any additional borrowing.

Text Table 3: Infrastructure Indicators in Selected Countries

\begin{tabular}{|c|c|c|c|c|c|c|}
\hline & $\begin{array}{l}\text { Households } \\
\text { w/ fixed } \\
\text { telephone }\end{array}$ & $\begin{array}{l}\text { Mobile } \\
\text { phones }^{2}\end{array}$ & $\begin{array}{l}\text { House holds w/ } \\
\text { electricity }{ }^{1}\end{array}$ & Roads & $\begin{array}{l}\text { Access to } \\
\text { improved } \\
\text { sanitation }{ }^{1} w\end{array}$ & $\begin{array}{c}\text { Access to } \\
\text { improved } \\
\text { water source }{ }^{1}\end{array}$ \\
\hline & $\begin{array}{c}\text { percent of } \\
\text { households }\end{array}$ & $\begin{array}{l}\text { Subscribers } \\
\text { per } 100 \\
\text { people }\end{array}$ & $\begin{array}{c}\text { percent } \\
\text { connected to } \\
\text { network }\end{array}$ & $\begin{array}{c}\mathrm{Km} \text { per } 1,000 \\
\mathrm{~km}^{2} \text { of land }\end{array}$ & \multicolumn{2}{|c|}{ percent of the population } \\
\hline Tanzania & 10 & 21 & 11 & 62 & 33 & 55 \\
\hline Ghana & 8 & 32 & 44 & 187 & 10 & 80 \\
\hline Kenya & 12 & 30 & 13 & 111 & 42 & 57 \\
\hline Rwanda & 1 & 7 & 5 & 568 & 23 & 65 \\
\hline South Africa & 27 & 92 & 63 & 300 & 59 & 93 \\
\hline Uganda & 3 & 18 & 8 & 385 & 33 & 64 \\
\hline Zambia & 4 & 21 & 20 & 50 & 52 & 58 \\
\hline SSA & 7 & 16 & 29 & $\ldots$ & 31 & 58 \\
\hline SSA low income ${ }^{3}$ & 6 & 19 & 26 & $\ldots$ & 15 & 25 \\
\hline SSA middle income ${ }^{3}$ & 19 & 36 & 55 & $\ldots$ & 41 & 66 \\
\hline
\end{tabular}

Source: World Bank Africa Infrastructure Country Diagnotic Database and World Development Indicators.

${ }^{1}$ Primary source demographic and health surveys, latest available year for the period 2001-2008.

${ }^{2}$ As of 2006.

${ }^{3}$ Income groups are based on the World Bank's classification. 


\section{Revenue}

\section{Despite the sizeable improvements in revenue collection in recent years,} revenues remain below estimates of potential. Existing efforts to harmonize tax rates within the EAC limit the scope for unilateral adjustment of rates, but staff identified a range of options for broadening the tax base and bringing revenues closer towards potential (Box 4). In the absence of new measures, revenues are projected to increase modestly over the next few years as the economy strengthens, to 16.3 percent of GDP in 2010/11, and further to 16.9 percent by 2012/13, well below the potential of around 21 percent of GDP. Specific measures to bring revenue collection toward potential will be discussed for the second and third program years, at the time of the second program review. The authorities are considering measures to widen the tax base to capture more of the informal sector and administrative improvements to manage tax exemptions, starting with recently-passed legislation on the mining regime. 


\section{Box 4. Tax Revenue Improvements-Policy Options}

Tanzania's tax revenue-to-GDP ratio has risen sharply, reaching 15 percent of GDP in 2008/09 compared to less than 10 percent a decade earlier. The improvement largely reflects administrative reforms, with few adjustments in tax rates which are mostly harmonized with the other EAC members. Despite the improvement, estimates of tax potential suggest significant room for additional gains, potentially an additional 5-6 percent of GDP.

Key challenges in the period ahead include: concluding a revenue-sharing arrangement for the EAC common market (scheduled for July 1, 2010); the implications of an agriculture-based growth strategy for the evolution of

\begin{tabular}{ccccc}
\multicolumn{6}{c}{ Actual vs. Potential Tax-to-GDP Ratios for Selected African Countries } \\
$\begin{array}{cccc}\text { (In percent of GDP) } \\
\text { Country }\end{array}$ & Year & $\begin{array}{c}\text { Tax Revenue } \\
\text { (A) }\end{array}$ & $\begin{array}{c}\text { Potential Tax } \\
\text { Revenue (B) }\end{array}$ & Tax Gap (B-A) \\
\hline Kenya & 2005 & 18.6 & 20.6 & 2.0 \\
South Africa & 2005 & 27.4 & 27.0 & -0.4 \\
Tanzania & 2005 & 11.2 & 20.5 & 9.3 \\
Uganda & 2008 & 15.0 & 20.9 & 5.9 \\
\hline Source: World Economic Outlook, IMF; Staff estimates & 19.5 & 7.8 \\
\hline
\end{tabular}
the revenue-to-GDP ratio; dealing with a large and growing mining sector; reining in exemptions; and resisting tax competition within the EAC. Reform options that should be considered include:

\section{Near-term revenue measures}

- Increase excise tax rates to the levels in neighboring countries such as Kenya and Uganda.

- Reduce VAT exemptions and zero-ratings to broaden the effective tax base, reduce administrative burden, and thereby help close a potential loophole in the tax system.

- Hold the Personal Income Tax threshold constant in nominal terms for several years to broaden the income tax base (87 percent of workers exempted in 2005/06).

- Raise the fuel levy. Proceeds are earmarked for road construction and maintenance so that road users contribute directly to the increased government spending envisaged in these areas.

\section{Medium-term revenue measures}

- Tighten the mining regime, while grandfathering existing agreements. The Mining Act of 2010 raises royalties on all minerals except diamonds from 3 percent of "netback value" (value after deducting certain costs) to 4-5 percent of gross market value of the minerals produced. The Act also limits discretionary provision of tax benefits by applying general tax rules to all future stabilization contracts in the mining sector. In addition to these recent changes, consideration should be given to: (i) imposing withholding taxes on interest paid on foreign currency loans; (ii) limiting the deductibility of debt financing for income taxes; (iii) tightening provisions for investment allowances for exploration and development; and (iv) ring-fencing income tax bases by mines. 


\section{Borrowing}

\section{The authorities plan to supplement available concessional resources with} nonconcessional borrowing of $\$ 1.5$ billion over the three year period of the PSI, roughly evenly phased over three years. Concessional borrowing and grants will remain the main source of financing, and the authorities will provide exemptions to their own laws on minimum concessionality after carefully examining the rate of return and feasibility of each specific project. The authorities have identified priority projects to be financed within the ceiling, focusing on transportation_roads, airports, railways, and ports - many of which are components of regional transportation networks, and which constitute a subset of the highest priority projects identified in the medium-term public investment plan (MPIP). ${ }^{3}$ Potential sources of funding are being explored, but one option under consideration is a Eurobond issuance once appropriate preparations have been made - indeed an advisory firm is being recruited to advance such preparations, including establishing a sovereign credit rating.

\section{An updated Bank-Fund DSA suggests scope for the new borrowing without} breaching DSA risk thresholds (Supplement I). Extensive debt relief in recent years has greatly reduced the external debt burden, providing room for scaling up growth-enhancing spending. The long-term outlook under the DSA baseline scenario, including increased borrowing to finance the aforementioned infrastructure investment and further unspecified investment of 1 percent of GDP from 2013 onward, shows a modest increase in debt indicators compared to the most recent Bank/Fund DSA, but the indicators would remain well below risk thresholds. However, borrowing on more expensive terms places additional importance on building institutional capacity to mitigate associated risks and preserve debt sustainability. The authorities are building capacity in these areas and expect to have elaborated a new Medium-Term Debt Strategy by end-December that would also include contingent liabilities arising under PPPs. The DSA conservatively assumes that the new borrowing is all on market terms, which would significantly increase the debt service burden and constrain future budgets.

\section{Ensuring returns to higher spending}

24. Strengthened public financial management systems could ensure better value for money from public resources. A key area of weakness is the current weak linkages between strategic documents (such as the MKUKUTA), the MPIP and the medium-term expenditure framework with the annual budget process. Improving such linkages could generate a more

\footnotetext{
${ }^{3}$ Several assessments support the conclusion that inadequate infrastructure in Tanzania serves as a binding constraint to higher growth, including in the region, considering Tanzania's geographical advantage to provide transportation networks for landlocked neighboring countries (Challenges of African Growth: Opportunities Constraints and Strategic Directions, Ndulu et al, 2007 (World Bank); Tanzania: The Story of an African Transition, Nord et al, 2009 (IMF); and Creating Sustainable Fiscal Space for Infrastructure: The Case of Tanzania, Ter-Minassian et al, 2008 (IMF Working Paper 08/256)).
} 
predictable flow of funds to support the implementation of multi-year programs and enhance prospects for achieving policy goals. At the same time, cash management, commitment controls, and arrears monitoring need to be strengthened to ensure adherence to budget guidelines. The PSI envisages a series of reforms in these areas (MEFP, paragraphs 25-29).

25. The return on infrastructure spending is ultimately driven by the private sector response. Tanzania has slipped in the World Bank Doing Business Indicators rankings in recent years. A task force appointed by the President has developed a roadmap to address some of the weaknesses identified - for example in simplifying license and registration procedures - with a series of measures scheduled for parliamentary consideration in July.

\section{Financial Sector Deepening}

26. An action plan is under development to further financial sector reforms, drawing on the FSAP update conducted in September 2009. ${ }^{4}$ While the financial system has expanded in recent years as lending has grown rapidly from a low base, financial intermediation and access to finance remain low compared to regional peers. Key recommendations from the FSAP update include:

- Increase compliance with prudential limits and address gaps in data collection. Banking supervisory processes are not fully risk-based and enforcement remains inconsistent, resulting in mixed compliance with prudential requirements and widespread under-provisioning. Moreover, gaps in prudential data collection and analysis prevent a comprehensive assessment of systemic and individual risks in the financial sector.

- Take the necessary steps to operationalize the Social Security Regulatory Authority. The SSRA was nominally established in June 2008, but the Board and directorgeneral have yet to be appointed. A number of key measures, including the introduction of investment guidelines for pension funds and implementation of comprehensive governing legislation, have also been delayed as a result. Investment guidelines, supervision and monitoring of the pension sector are crucial, both to contain potential fiscal liabilities and to facilitate capital market development.

- Address remaining weaknesses in the crisis management framework. The current framework appears adequate for resolution of individual banks, but further work is needed to prepare for a broader systemic crisis, including detailed contingency plans and an explicit framework for systemic emergency liquidity assistance and recapitalization. In addition, the Deposit Insurance Fund remains underfunded and its reserves should be increased to enhance the DIF's ability to provide coverage in the event of the failure of a larger bank.

\footnotetext{
${ }^{4}$ Additional information is provided in the Financial System Stability Assessment (www.imf.org).
} 
27. The BoT is already taking steps to strengthen crisis preparedness. While the banking system was largely unaffected by the global crisis, it is important to ensure that appropriate systems are in place to facilitate a rapid response in the event that pressures emerge. Drawing on the FSAP recommendations, three key components are already underway:

- Minimum capital requirements are being raised by a factor of 3, bringing requirements to TSh 15 billion for commercial banks and TSh 7.5 billion for financial institutions;

- The size of the Deposit Insurance Fund is being increased, while the maximum insurance coverage has been raised from TSh 500,000 to TSh 1,500,000 per depositor per bank; and

- A framework for emergency liquidity assistance is being developed.

28. Preparations are underway to ensure the effectiveness of supervision over social security funds, once a regulator is appointed. The recruitment process for the regulator is expected to be concluded by June. In the meantime, new actuarial audits of each of the social security funds are in process, and the BoT is developing a reporting template to ensure the collection of data needed.

29. Capital account liberalization should be pursued gradually. While this is an essential component of the move to a common currency in the EAC, there are costs and benefits from opening up capital markets. On the positive side, the increased pool of financial resources could play an important role in expanding options for increased investment, both public and private. On the other hand, in addition to the potential for capital outflows, the authorities noted the risk of significantly increased volatility given relatively shallow domestic financial markets - indeed, the absence of sizable portfolio positions by foreign investors in domestic markets was an important factor in minimizing the impact of the global crisis. A time bound action plan for progressively lifting controls is under preparation. It was agreed that the legal steps needed to be complemented by ensuring progress on the economic and institutional prerequisites — such as developing secondary markets in debt instruments and establishing a system for monitoring cross-border portfolio flows.

\section{Program Modalities}

30. The PSI program period will begin on June 1, 2010, and assessment criteria, in line with those of the existing PSI-supported program/ESF arrangement, will be established for end-June and end-December 2010. The criteria are presented in Table 1 of the authorities' Memorandum of Economic and Financial Policies (MEFP, Appendix I, attachment I). A system is being established to enable regular monitoring of MKUKUTA related spending - currently the information is only available at the time of formulation of the budget - as well as a system to monitor on a quarterly basis the outstanding stock of uncleared payment claims at key line Ministries. The authorities' structural reform agenda, 
reflected in the proposed structural benchmarks during the first year of the PSI, includes: approval of MKUKUTA II and a social welfare framework; measures to strengthen public financial and debt management; the implementation of key FSAP recommendations, addressing the weaknesses in social security supervision; and strengthening the statistical database (MEFP, Appendix I, Table 3).

\section{Staff Appraisal}

31. The economy has weathered the impacts of the global crisis well - the macroeconomic policy stance, including fiscal stimulus and monetary easing, as well as the targeted and time bound measures contained in the economic rescue plan have helped ring fence the impact of the crisis and support the early recovery of growth. The recovery is still nascent and there are risks from both additional global shocks, as well as more localized events such as the recent drought and subsequent floods that created food security concerns and disrupted key regional infrastructure.

32. For FY2010/11, a careful balance is needed to begin reining in the fiscal stimulus without jeopardizing the economic recovery. A combination of a modest improvement in revenues as the economy strengthens and the expiration of discretionary measures introduced in FY09/10 will narrow the deficit, despite higher planned investment spending. Inflationary pressures have eased as the impacts of food supply shocks have dissipated. The uncertain timing of the recovery in private sector credit growth will require a continued careful monitoring of liquidity conditions to prevent an excessive build up of liquidity in the financial system that could fuel inflation or exchange rate pressures.

33. Staff recommends the completion of the $7^{\text {th }}$ review under the PSI and the $2^{\text {nd }}$ review of the ESF arrangement. All quantitative performance/assessment criteria have been observed and there has been reasonable progress on the structural reforms.

34. The proposed scaling-up of infrastructure investment could address an important constraint to growth, both for the country and the region. Ensuring that projects generate the intended returns will require careful planning, realistic assessments of capacity constraints, strong project implementation, and improvements in the business climate to enable a strong private sector response. The focus on agriculture and plans to establish a formal on-budget social safety net should help ensure that the growth gains are well-distributed and alleviate food security concerns.

35. Creating additional fiscal space to enable a scaling-up of infrastructure spending can be partly achieved through a combination of revenue measures and rationalization of existing expenditures. While significant gains in revenues have been attained in recent years, revenues remain well below potential and there is room for raising collections through aligning tax rates with the region and by broadening the tax base including by reining in exemptions. Recurrent spending has risen rapidly in recent years, particularly in the "other goods and services" category, and a careful examination of the need for and level of such expenditures could free up valuable resources. Commitment control at line Ministries and 
spending agencies should also be tightened to avoid arrears and ensure that budgets are implemented as intended.

36. The authorities' plans to supplement available grants and concessional lending with a modest amount of borrowing from quasi-concessional and market sources, as well as through greater involvement of the private sector, are consistent with the maintenance of a low risk of debt distress in the debt sustainability analysis. The strategy is not without risks. An updated medium-term debt strategy, including contingent liabilities, together with the approval of a formal policy and safeguards for contracting PPPs will be critical to containing risks.

37. The banking system appears well-capitalized, but the continued weaknesses in the supervision of the social security funds imparts a risk to the health of the broader financial system and prevents a deepening of capital markets. Increased compliance with provisioning requirements, better data collection, improved crisis management tools, and sufficient deposit insurance are also needed to contain risks. Initial steps to implement key recommendations from the FSAP in these areas are welcome.

38. Staff supports the authorities' request for a new PSI. While the new poverty reduction and growth strategy is still under development, the greater emphasis on growth while sustaining gains already achieved in social sectors can provide a platform for sustainable improvements in living standards and continued progress towards the MDGs. Implementation of the new strategy will be greatly assisted by a clear prioritization of objectives so that policy interventions can be linked directly to the MTEF, consistent with a credible resource envelope and predictable funding. 
Table 1. Tanzania: Selected Economic and Financial Indicators, 2007/08-2012/13

\begin{tabular}{|c|c|c|c|c|c|c|c|}
\hline & \multirow[t]{2}{*}{$2007 / 08$} & \multirow[t]{2}{*}{ 2008/09 } & \multicolumn{2}{|c|}{$2009 / 10$} & \multirow{2}{*}{$\begin{array}{r}\text { 2010/11 } \\
\text { Proj. }\end{array}$} & \multirow{2}{*}{$\begin{array}{r}2011 / 12 \\
\text { Proj. }\end{array}$} & \multirow{2}{*}{$\begin{array}{r}2012 / 13 \\
\text { Proj. }\end{array}$} \\
\hline & & & Prog. $^{5}$ & Proj. & & & \\
\hline & \multicolumn{7}{|c|}{ (Annual percentage change, unless otherwise indicated) } \\
\hline \multicolumn{8}{|l|}{ National income and prices } \\
\hline Real GDP growth (calendar year) ${ }^{1}$ & 7.1 & 7.4 & 5.0 & 5.5 & 6.2 & 6.7 & 7.5 \\
\hline Real GDP growth (fiscal year) & 7.3 & 6.4 & 5.5 & 5.8 & 6.5 & 7.1 & 7.5 \\
\hline Consumer prices (period average) & 8.4 & 11.8 & $8.0-9.0$ & 10.6 & 5.8 & 5.0 & 5.0 \\
\hline Consumer prices (end of period) & 9.3 & 10.7 & $6.0-8.0$ & 8.0 & 5.0 & 5.0 & 5.0 \\
\hline \multicolumn{8}{|l|}{ External sector } \\
\hline Export, f.o.b (in millions of U.S. dollars) & 2,609 & 2,969 & 3,154 & 3,216 & 3,826 & 4,168 & 4,565 \\
\hline Imports, f.o.b. (in millions of U.S. dollars) & $-5,667$ & $-5,970$ & $-6,070$ & $-6,149$ & $-6,996$ & $-7,539$ & $-8,045$ \\
\hline Export volume & 6.2 & 5.3 & 6.2 & 6.6 & 10.3 & 9.8 & 8.4 \\
\hline Import volume & 13.3 & -1.5 & 8.9 & 6.0 & 6.4 & 5.6 & 4.3 \\
\hline Terms of trade & -7.7 & 10.9 & 7.7 & 8.2 & -2.6 & -2.7 & -1.2 \\
\hline Nominal effective exchange rate (end of period; depreciation -) & 1.8 & -3.4 & $\ldots$ & $\ldots$ & $\ldots$ & $\ldots$ & $\ldots$ \\
\hline Real effective exchange rate (end of period; depreciation -) & 2.9 & 6.4 & $\ldots$ & $\ldots$ & $\ldots$ & $\ldots$ & $\ldots$ \\
\hline \multicolumn{8}{|l|}{ Money and credit ${ }^{2}$} \\
\hline Broad money (M3) & 18.1 & 19.0 & 21.1 & 21.6 & 21.0 & $\ldots$ & $\ldots$ \\
\hline Net foreign assets & 4.7 & 15.7 & -5.2 & 13.1 & 13.4 & $\ldots$ & $\ldots$ \\
\hline Net domestic assets & 39.8 & 23.0 & 51.2 & 31.2 & 28.5 & $\ldots$ & $\ldots$ \\
\hline Credit to nongovernment sector & 32.9 & 33.6 & 33.6 & 14.1 & 21.3 & $\ldots$ & $\ldots$ \\
\hline Velocity of money (GDP/M3; average) & 3.7 & 3.7 & 3.5 & 3.3 & 3.3 & $\ldots$ & $\ldots$ \\
\hline Treasury bill interest rate (in percent; end of period) ${ }^{3}$ & 7.8 & 7.0 & $\ldots$ & 4.5 & $\ldots$ & $\ldots$ & $\ldots$ \\
\hline & \multicolumn{7}{|c|}{ (Percent of GDP) } \\
\hline Public Finance & & & & & & & \\
\hline Revenue (excluding grants) & 15.9 & 15.8 & 16.5 & 15.8 & 16.3 & 16.6 & 16.9 \\
\hline Total grants & 6.9 & 4.9 & 6.2 & 6.3 & 4.9 & 4.6 & 4.3 \\
\hline Expenditure & 22.8 & 25.5 & 27.6 & 27.7 & 27.1 & 26.5 & 25.8 \\
\hline Overall balance (excluding grants) & -6.9 & -9.6 & -11.1 & -12.0 & -10.8 & -10.0 & -8.9 \\
\hline Domestic financing & -1.5 & 0.8 & 1.6 & 1.6 & 1.0 & 1.0 & 1.0 \\
\hline Stock of domestic debt (end of period) & 13.4 & 12.0 & 12.0 & 11.9 & 11.7 & 11.4 & 11.1 \\
\hline \multicolumn{8}{|l|}{ Savings and investment ${ }^{1}$} \\
\hline Resource gap & -16.9 & -13.7 & -12.2 & -12.1 & -11.3 & -11.2 & -10.7 \\
\hline Investment & 29.6 & 29.8 & 28.4 & 27.8 & 28.0 & 28.6 & 28.9 \\
\hline Government & 9.7 & 10.0 & 10.4 & 10.3 & 10.6 & 10.3 & 9.5 \\
\hline Nongovernment ${ }^{4}$ & 20.0 & 19.8 & 18.0 & 17.5 & 17.4 & 18.4 & 19.5 \\
\hline Gross domestic savings & 12.8 & 16.2 & 16.2 & 15.7 & 16.7 & 17.4 & 18.3 \\
\hline \multicolumn{8}{|l|}{ External sector } \\
\hline Current account balance (excluding current transfers) & -14.1 & -13.1 & -11.6 & -11.5 & -11.7 & -11.7 & -11.1 \\
\hline Current account balance (including current transfers) & -10.6 & -9.8 & -8.6 & -8.3 & -9.3 & -9.4 & -9.0 \\
\hline & \multicolumn{7}{|c|}{ (Millions of U.S. dollars, unless otherwise indicated) } \\
\hline \multicolumn{8}{|l|}{ Balance of payments } \\
\hline Current account balance (excluding current transfers; deficit -) & $-2,675$ & $-2,804$ & $-2,760$ & $-2,695$ & $-2,994$ & $-3,240$ & $-3,384$ \\
\hline Gross official reserves & 2,660 & 2,925 & 3,277 & 3,461 & 3,755 & 4,085 & 4,365 \\
\hline In months of imports of goods and nonfactor services (current year) & 4.5 & 4.6 & 5.1 & 5.2 & 5.0 & 5.0 & 5.0 \\
\hline External debt (percent of GDP) & 24.9 & 25.6 & 25.3 & 26.6 & 29.5 & 32.0 & 33.6 \\
\hline
\end{tabular}

Sources: Tanzanian authorities; and Fund staff estimates and projections.

${ }^{1}$ Data are on calendar year basis. For example, 2007/08 data are for calendar year 2007.

${ }^{2}$ Figures for 2008/09 onward reflect a new series based on the Fund's 2000 Monetary and Financial Statistics Manual.

${ }^{3}$ End-year (June) monthly weighted-average yield of 35-, 91-, 182-, and 364-day treasury bills. For 2009/10, figure is for March 2010.

${ }^{4}$ Including change in stocks.

${ }^{5}$ From the sixth review under the PSI, based on previous GDP projection. 
Table 2a. Tanzania: Central Government Operations, 2007/08-2012/13 ${ }^{1}$ (Billions of Tanzania Shillings)

\begin{tabular}{|c|c|c|c|c|c|c|c|}
\hline & \multirow[t]{2}{*}{$2007 / 08$} & \multirow[t]{2}{*}{$2008 / 09$} & \multicolumn{2}{|c|}{$2009 / 10$} & \multirow{2}{*}{$\begin{array}{r}\text { 2010/11 } \\
\text { Proj. }\end{array}$} & \multirow{2}{*}{$\begin{array}{r}2011 / 12 \\
\text { Proj. }\end{array}$} & \multirow{2}{*}{$\begin{array}{r}2012 / 13 \\
\text { Proj. }\end{array}$} \\
\hline & & & Budget & Proj. $^{6}$ & & & \\
\hline Total revenue & 3,635 & 4,293 & 5,234 & 4,933 & 5,686 & 6,495 & 7,462 \\
\hline Tax revenue & 3,359 & 4,044 & 4,841 & 4,577 & 5,252 & 5,999 & 6,857 \\
\hline Import duties & 289 & 359 & 410 & 390 & 457 & 522 & 597 \\
\hline Value-added tax & 1,042 & 1,231 & 1,474 & 1,467 & 1,674 & 1,912 & 2,186 \\
\hline Excises & 661 & 762 & 975 & 876 & 1,006 & 1,149 & 1,313 \\
\hline Income taxes & 984 & 1,229 & 1,428 & 1,338 & 1,536 & 1,754 & 2,005 \\
\hline Other taxes & 383 & 463 & 553 & 505 & 580 & 662 & 757 \\
\hline Nontax revenue $^{2}$ & 275 & 249 & 393 & 356 & 434 & 495 & 604 \\
\hline Total expenditure & 5,217 & 6,907 & 8,862 & 8,685 & 9,464 & 10,406 & 11,404 \\
\hline Recurrent expenditure & 3,398 & 4,681 & 6,037 & 5,946 & 6,119 & 6,744 & 7,509 \\
\hline Wages and salaries & 1,135 & 1,609 & 1,766 & 1,774 & 2,116 & 2,375 & 2,685 \\
\hline Interest payments & 265 & 243 & 408 & 367 & 388 & 514 & 574 \\
\hline Domestic & 237 & 208 & 355 & 314 & 336 & 368 & 358 \\
\hline Foreign $^{3}$ & 27 & 35 & 53 & 53 & 51 & 146 & 215 \\
\hline Goods and services and transfers ${ }^{2}$ & 1,998 & 2,830 & 3,863 & 3,805 & 3,615 & 3,854 & 4,250 \\
\hline of which: MDRI (IMF) related & 7 & 0 & 0 & 0 & 0 & 0 & 0 \\
\hline Development expenditure & 1,819 & 2,226 & 2,825 & 2,739 & 3,345 & 3,663 & 3,895 \\
\hline Domestically financed & 567 & 906 & 968 & 968 & 1,517 & 1,823 & 1,918 \\
\hline of which: MDRI (IMF) related & 114 & 68 & 131 & 131 & 0 & 0 & 0 \\
\hline Foreign financed & 1,252 & 1,320 & 1,857 & 1,770 & 1,827 & 1,840 & 1,977 \\
\hline Overall balance before grants & $-1,583$ & $-2,614$ & $-3,628$ & $-3,752$ & $-3,778$ & $-3,912$ & $-3,943$ \\
\hline Grants & 1,581 & 1,340 & 2,091 & 1,972 & 1,729 & 1,812 & 1,898 \\
\hline Program (including basket grants) ${ }^{4}$ & 832 & 798 & 1,108 & 989 & 834 & 880 & 925 \\
\hline Project & 636 & 462 & 852 & 852 & 895 & 932 & 973 \\
\hline MDRI (IMF) grant relief & 114 & 80 & 131 & 131 & 0 & 0 & 0 \\
\hline Overall balance after grants & -1 & $-1,275$ & $-1,537$ & $-1,780$ & $-2,048$ & $-2,100$ & $-2,045$ \\
\hline Adjustment to cash $^{5}$ & -365 & 60 & 0 & 0 & 0 & 0 & 0 \\
\hline Overall balance (cash basis) & -366 & $-1,215$ & $-1,537$ & $-1,780$ & $-2,048$ & $-2,100$ & $-2,045$ \\
\hline Financing gap & 0 & 0 & 0 & 0 & 0 & 0 & 0 \\
\hline Financing & 366 & 1,215 & 1,537 & 1,780 & 2,048 & 2,100 & 2,045 \\
\hline Foreign (net) & 730 & 956 & 1,037 & 1,280 & 1,699 & 1,708 & 1,602 \\
\hline Foreign loans & 775 & 984 & 1,091 & 1,324 & 1,785 & 1,810 & 1,778 \\
\hline Program (including basket loans) ${ }^{4}$ & 566 & 495 & 659 & 892 & 607 & 592 & 622 \\
\hline Project & 209 & 489 & 432 & 432 & 454 & 477 & 501 \\
\hline Nonconcessional borrowing & & 0 & 0 & 0 & 724 & 741 & 655 \\
\hline Amortization & -45 & -27 & -54 & -44 & -86 & -102 & -176 \\
\hline Domestic (net) & -346 & 214 & 506 & 506 & 350 & 392 & 443 \\
\hline Bank financing & $\ldots$ & $\ldots$ & $\ldots$ & 466 & 310 & 342 & 393 \\
\hline Nonbank financing & $\ldots$ & $\ldots$ & $\ldots$ & 40 & 40 & 50 & 50 \\
\hline Amortization of parastatal debt & -15 & 0 & -21 & -21 & 0 & 0 & 0 \\
\hline Privatization proceeds & 0 & 45 & 15 & 15 & 0 & 0 & 0 \\
\hline \multicolumn{8}{|l|}{ Memorandum items: } \\
\hline Public domestic debt (in percent of GDP) & 13.3 & 12.0 & 12.2 & 11.9 & 11.7 & 11.4 & 11.1 \\
\hline Recurrent expenditures in pecent of total revenues & 93 & 109 & 115 & 121 & 108 & 104 & 101 \\
\hline Nominal GDP & 22,851 & 27,132 & 31,109 & 31,299 & 34,970 & 39,219 & 44,257 \\
\hline
\end{tabular}

Sources: Ministry of Finance; Bank of Tanzania; and Fund staff projections.

${ }^{1}$ Fiscal year: July-June.

${ }^{2}$ In 2008/09, nontax revenue include the recovery from a fraudulent payment made from the government's EPA account managed by the Bank of Tanzania. Local Government Authorities' own revenues (about 0.5 percent of GDP), and the equal amount of transfers, are included starting from FY2009/10.

${ }^{3}$ Some projected external debt obligations are under negotiation for relief with a number of creditors.

${ }^{4}$ Basket funds are sector-specific accounts established by the government to channel donor support to fund-specific activities.

${ }^{5}$ Unidentified financing $(+) /$ expenditure (-). Includes expenditure carryover from the previous year.

${ }^{6}$ Based on revised GDP. 
Table 2b. Tanzania: Central Government Operations, 2007/08- 2012/13 (Percent of GDP)

\begin{tabular}{|c|c|c|c|c|c|c|c|}
\hline & \multirow[t]{2}{*}{$2007 / 08$} & \multirow[t]{2}{*}{$2008 / 09$} & \multicolumn{2}{|c|}{$2009 / 10$} & \multirow{2}{*}{$\frac{2010 / 11}{\text { Proj. }}$} & \multirow{2}{*}{$\frac{2011 / 12}{\text { Proj. }}$} & \multirow{2}{*}{$\frac{2012 / 13}{\text { Proj. }}$} \\
\hline & & & Budget & Proj. ${ }^{6}$ & & & \\
\hline Total revenue & 15.9 & 15.8 & 16.8 & 15.8 & 16.3 & 16.6 & 16.9 \\
\hline Tax revenue & 14.7 & 14.9 & 15.6 & 14.6 & 15.0 & 15.3 & 15.5 \\
\hline Import duties & 1.3 & 1.3 & 1.3 & 1.2 & 1.3 & 1.3 & 1.3 \\
\hline Value-added tax & 4.6 & 4.5 & 4.7 & 4.7 & 4.8 & 4.9 & 4.9 \\
\hline Excises & 2.9 & 2.8 & 3.1 & 2.8 & 2.9 & 2.9 & 3.0 \\
\hline Income taxes & 4.3 & 4.5 & 4.6 & 4.3 & 4.4 & 4.5 & 4.5 \\
\hline Other taxes & 1.7 & 1.7 & 1.8 & 1.6 & 1.7 & 1.7 & 1.7 \\
\hline Nontax revenue $^{2}$ & 1.2 & 0.9 & 1.3 & 1.1 & 1.2 & 1.3 & 1.4 \\
\hline Total expenditure & 22.8 & 25.5 & 28.5 & 27.7 & 27.1 & 26.5 & 25.8 \\
\hline Recurrent expenditure & 14.9 & 17.3 & 19.4 & 19.0 & 17.5 & 17.2 & 17.0 \\
\hline Wages and salaries & 5.0 & 5.9 & 5.7 & 5.7 & 6.1 & 6.1 & 6.1 \\
\hline Interest payments & 1.2 & 0.9 & 1.3 & 1.2 & 1.1 & 1.3 & 1.3 \\
\hline Domestic & 1.0 & 0.8 & 1.1 & 1.0 & 1.0 & 0.9 & 0.8 \\
\hline Foreign $^{3}$ & 0.1 & 0.1 & 0.2 & 0.2 & 0.1 & 0.4 & 0.5 \\
\hline Goods and services and transfers ${ }^{2}$ & 8.7 & 10.4 & 12.4 & 12.2 & 10.3 & 9.8 & 9.6 \\
\hline of which: MDRI (IMF) related & 0.0 & 0.0 & 0.0 & 0.0 & 0.0 & 0.0 & 0.0 \\
\hline Development expenditure & 8.0 & 8.2 & 9.1 & 8.7 & 9.6 & 9.3 & 8.8 \\
\hline Domestically financed & 2.5 & 3.3 & 3.1 & 3.1 & 4.3 & 4.6 & 4.3 \\
\hline of which: MDRI (IMF) related & 0.5 & 0.3 & 0.4 & 0.4 & 0.0 & 0.0 & 0.0 \\
\hline Foreign financed & 5.5 & 4.9 & 6.0 & 5.7 & 5.2 & 4.7 & 4.5 \\
\hline Overall balance before grants & -6.9 & -9.6 & -11.7 & -12.0 & -10.8 & -10.0 & -8.9 \\
\hline Grants & 6.9 & 4.9 & 6.7 & 6.3 & 4.9 & 4.6 & 4.3 \\
\hline Program (including basket grants) ${ }^{4}$ & 3.6 & 2.9 & 3.6 & 3.2 & 2.4 & 2.2 & 2.1 \\
\hline Project & 2.8 & 1.7 & 2.7 & 2.7 & 2.6 & 2.4 & 2.2 \\
\hline MDRI (IMF) grant relief & 0.5 & 0.3 & 0.4 & 0.4 & 0.0 & 0.0 & 0.0 \\
\hline Overall balance after grants & 0.0 & -4.7 & -4.9 & -5.7 & -5.9 & -5.4 & -4.6 \\
\hline Adjustment to cash ${ }^{5}$ & -1.6 & 0.2 & 0.0 & 0.0 & 0.0 & 0.0 & 0.0 \\
\hline Overall balance (cash basis) & -1.6 & -4.5 & -4.9 & -5.7 & -5.9 & -5.4 & -4.6 \\
\hline Financing gap & & 0.0 & 0.0 & 0.0 & 0.0 & 0.0 & 0.0 \\
\hline Financing & 1.6 & 4.5 & 4.9 & 5.7 & 5.9 & 5.4 & 4.6 \\
\hline Foreign (net) & 3.2 & 3.5 & 3.3 & 4.1 & 4.9 & 4.4 & 3.6 \\
\hline Foreign loans & 3.4 & 3.6 & 3.5 & 4.2 & 5.1 & 4.6 & 4.0 \\
\hline Program (including basket loans) ${ }^{4}$ & 2.5 & 1.8 & 2.1 & 2.8 & 1.7 & 1.5 & 1.4 \\
\hline Project & 0.9 & 1.8 & 1.4 & 1.4 & 1.3 & 1.2 & 1.1 \\
\hline Nonconcessional borrowing & & 0.0 & 0.0 & 0.0 & 2.1 & 1.9 & 1.5 \\
\hline Amortization & -0.2 & -0.1 & -0.2 & -0.1 & -0.2 & -0.3 & -0.4 \\
\hline Domestic (net) & -1.5 & 0.8 & 1.6 & 1.6 & 1.0 & 1.0 & 1.0 \\
\hline Bank financing & $\ldots$ & $\ldots$ & $\ldots$ & 1.5 & 0.9 & 0.9 & 0.9 \\
\hline Nonbank financing & $\ldots$ & $\ldots$ & $\ldots$ & 0.1 & 0.1 & 0.1 & 0.1 \\
\hline Amortization of parastatal debt & -0.1 & 0.0 & -0.1 & -0.1 & 0.0 & 0.0 & 0.0 \\
\hline Privatization proceeds & 0.0 & 0.2 & 0.0 & 0.0 & 0.0 & 0.0 & 0.0 \\
\hline
\end{tabular}

Sources: Ministry of Finance; Bank of Tanzania; and Fund staff projections.

${ }^{1}$ Fiscal year: July-June.

${ }^{2}$ In 2008/09, nontax revenue include the recovery from a fraudulent payment made from the government's EPA account managed by the Bank of Tanzania. Local Government Authorities' own revenues (about 0.5 percent of GDP), and the equal amount of transfers, are included starting from FY2009/10.

${ }^{3}$ Some projected external debt obligations are under negotiation for relief with a number of creditors.

${ }^{4}$ Basket funds are sector-specific accounts established by the government to channel donor support to fund-specific activities.

${ }^{5}$ Unidentified financing (+)/expenditure (-). Includes expenditure carryover from the previous year.

${ }^{6}$ Based on revised GDP. 
Table 3. Tanzania: Monetary accounts, 2007/08 - 2010/11

(Billions of Tanzania Shillings, unless otherwise indicated; end of period)

\begin{tabular}{|c|c|c|c|c|}
\hline & $2007 / 08$ & $2008 / 09$ & $\begin{array}{r}\text { 2009/10 } \\
\text { Proj. }\end{array}$ & $\begin{array}{r}2010 / 11 \\
\text { Proj. }\end{array}$ \\
\hline \multicolumn{5}{|l|}{ Bank of Tanzania } \\
\hline Net foreign assets & 3,060 & 3,395 & 3,736 & 4,258 \\
\hline Net international reserves & 3,120 & 3,458 & 4,156 & 4,691 \\
\hline (Millions of U.S. dollars) & 2,642 & 2,661 & 3,079 & 3,374 \\
\hline Net non-reserve foreign assets & -60 & -63 & -420 & -433 \\
\hline Net domestic assets & -980 & -716 & -494 & -416 \\
\hline Credit to government & $-1,407$ & -779 & -622 & -537 \\
\hline of which: Excluding counterpart of liquidity paper & -240 & 212 & 478 & 503 \\
\hline Other items (net) & 427 & 63 & 128 & 121 \\
\hline REPOs & -105 & -131 & -110 & -97 \\
\hline Other items, excluding REPOs (net) & 532 & 194 & 238 & 218 \\
\hline of which: Credit to nongovernment sector & 79 & 55 & 101 & 101 \\
\hline Reserve money ${ }^{1}$ & 2,080 & 2,679 & 3,242 & 3,841 \\
\hline Currency outside banks & 1,269 & 1,424 & 1,628 & 1,887 \\
\hline Bank reserves & 810 & 1,255 & 1,614 & 1,954 \\
\hline Currency in banks & 182 & 259 & 334 & 442 \\
\hline Deposits & 628 & 996 & 1,280 & 1,513 \\
\hline Required reserves (calculated) ${ }^{1}$ & 504 & 787 & 926 & 1,101 \\
\hline Excess reserves (calculated) & 124 & 209 & 354 & 411 \\
\hline \multicolumn{5}{|l|}{ Memorandum items: } \\
\hline Stock of liquidity paper & 1,168 & 990 & 1,100 & 1,040 \\
\hline Average reserve money & 2,054 & 2,602 & 3,161 & 3,746 \\
\hline \multicolumn{5}{|l|}{ Monetary Survey } \\
\hline Net foreign assets & 3,629 & 4,197 & 4,749 & 5,384 \\
\hline Bank of Tanzania & 3,060 & 3,395 & 3,736 & 4,258 \\
\hline Commercial banks & 569 & 802 & 1,013 & 1,126 \\
\hline Net domestic assets & 2,983 & 3,669 & 4,814 & 6,186 \\
\hline Domestic credit & 3,193 & 4,528 & 5,642 & 7,038 \\
\hline Credit to government (net) & -151 & 62 & 528 & 837 \\
\hline Credit to nongovernment sector & 3,344 & 4,467 & 5,114 & 6,201 \\
\hline Other items (net) & -210 & -859 & -828 & -853 \\
\hline M3 & 6,612 & 7,866 & 9,563 & 11,570 \\
\hline Foreign currency deposits & 1,785 & 2,098 & 2,486 & 3,031 \\
\hline M2 & 4,827 & 5,768 & 7,076 & 8,538 \\
\hline Currency in circulation & 1,269 & 1,424 & 1,628 & 1,887 \\
\hline Deposits (TSh) & 3,557 & 4,344 & 5,449 & 6,651 \\
\hline Memorandum items: & \multicolumn{4}{|c|}{ (12-month percent change, unless otherwise indicated) } \\
\hline M3 growth & 18.1 & 19.0 & 21.6 & 21.0 \\
\hline M2 growth & 26.6 & 19.5 & 22.7 & 20.7 \\
\hline Currency in circulation & 14.3 & 12.2 & 14.3 & 15.9 \\
\hline Credit to nongovernment & 32.9 & 33.6 & 14.5 & 21.3 \\
\hline Average reserve money & 21.9 & 26.7 & 21.5 & 18.5 \\
\hline Currency/M3 (in percent) & 22.0 & 21.4 & 20.5 & 20.1 \\
\hline
\end{tabular}

Sources: Bank of Tanzania; and Fund staff estimates and projections.

${ }^{1}$ In January 2009 the reserve requirement on government deposits was increased to 20 percent (from 10 percent) and cash in banks was no longer counted towards required reserves. Previously, 50 percent of cash in banks was counted toward required reserves.

\section{CInternational Monetary Fund. Not for Redistribution}


Table 4. Tanzania: Summary Accounts of the Bank of Tanzania, 2009/10-2010/11 (Billions of Tanzania Shillings, unless otherwise indicated; end of period)

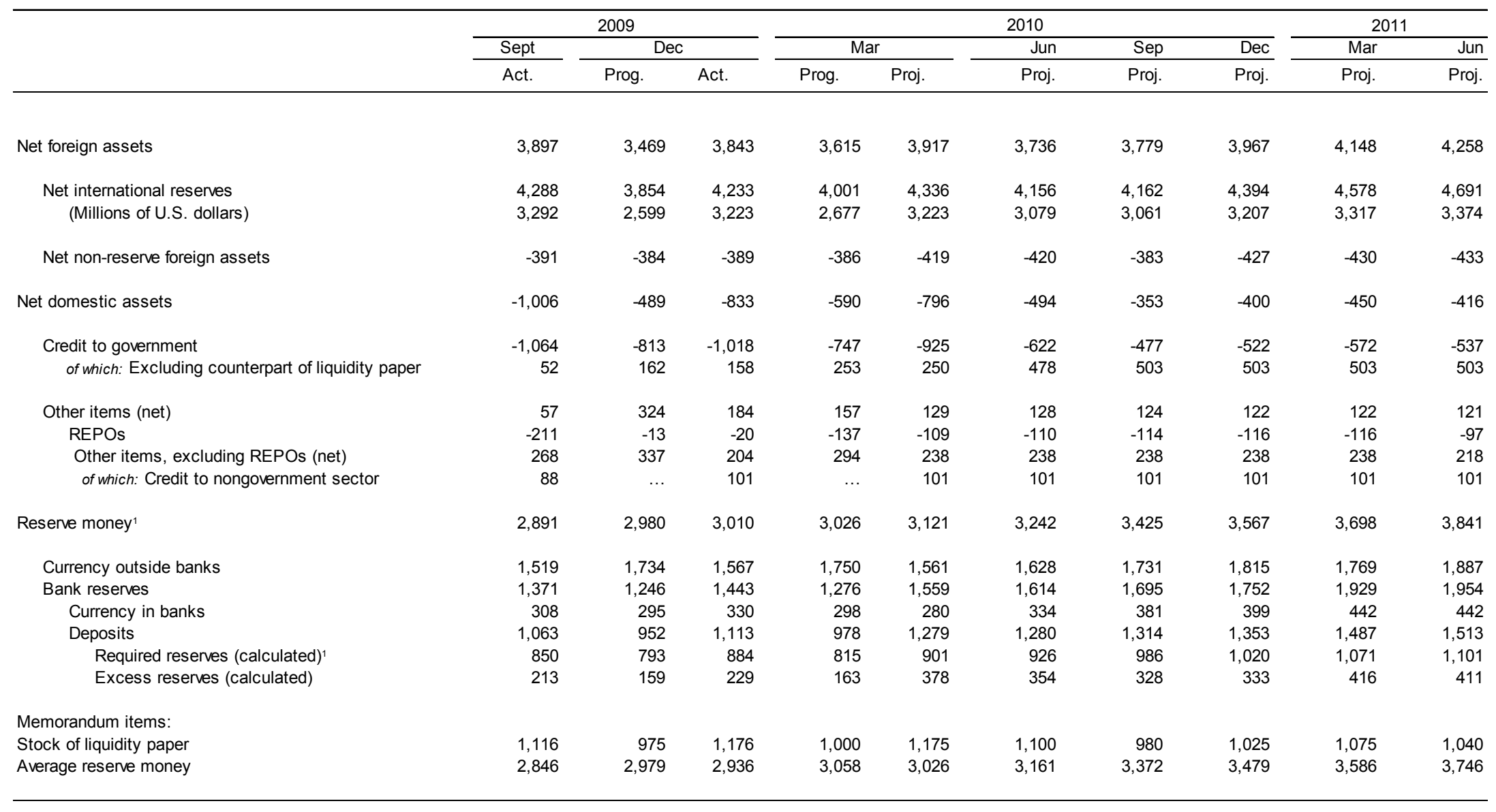

Sources: Bank of Tanzania; and Fund staff estimates and projections.

${ }^{1}$ In January 2009 the reserve requirement on government deposits was increased to 20 percent (from 10 percent) and vault cash in banks was no longer counted towards required reserves. Previously, 50 percent of vault cash in banks was counted toward required reserves. 
Table 5. Tanzania: Monetary Survey, 2009/10-2010/11

(Billions of Tanzania Shillings, unless otherwise indicated; end of period)

\begin{tabular}{|c|c|c|c|c|c|c|c|c|c|c|c|}
\hline & \multicolumn{4}{|c|}{2009} & \multicolumn{5}{|c|}{2010} & \multicolumn{2}{|c|}{2011} \\
\hline & \multicolumn{2}{|c|}{ Sep } & \multicolumn{2}{|c|}{ Dec } & \multicolumn{2}{|c|}{ Mar } & \multirow{2}{*}{$\begin{array}{r}\text { Jun } \\
\text { Proj. }\end{array}$} & \multirow{2}{*}{$\begin{aligned} \text { Sep } \\
\text { Proj. }\end{aligned}$} & \multirow{2}{*}{$\begin{array}{r}\text { Dec } \\
\text { Proj. }\end{array}$} & \multirow{2}{*}{$\begin{array}{c}\text { Mar } \\
\text { Proj. }\end{array}$} & \multirow{2}{*}{$\begin{array}{r}\text { Jun } \\
\text { Proj }\end{array}$} \\
\hline & Prog. & Act. & Prog. & Act. & Prog. & Proj. & & & & & \\
\hline Net foreign assets & 3,673 & 4,864 & 3,944 & 4,938 & 4,103 & 4,973 & 4,749 & 4,799 & 5,049 & 5,349 & 5,384 \\
\hline Bank of Tanzania & 3,210 & 3,897 & 3,469 & 3,843 & 3,615 & 3,917 & 3,736 & 3,779 & 3,967 & 4,148 & 4,258 \\
\hline Commercial banks & 463 & 967 & 475 & 1,095 & 488 & 1,056 & 1,013 & 1,020 & 1,083 & 1,201 & 1,126 \\
\hline Net domestic assets & 4,959 & 3,612 & 5,131 & 3,894 & 5,215 & 4,232 & 4,814 & 5,460 & 5,633 & 5,800 & 6,186 \\
\hline Domestic credit & 5,349 & 4,504 & 5,567 & 4,812 & 5,699 & 5,057 & 5,642 & 6,214 & 6,473 & 6,646 & 7,038 \\
\hline Credit to government (net) & 390 & -80 & 194 & 76 & 256 & 200 & 528 & 778 & 729 & 748 & 837 \\
\hline Credit to nongovernment sector & 4,959 & 4,584 & 5,373 & 4,736 & 5,443 & 4,858 & 5,114 & 5,437 & 5,745 & 5,898 & 6,201 \\
\hline Other items (net) & -391 & -892 & -436 & -918 & -484 & -825 & -828 & -754 & -840 & -846 & -853 \\
\hline M3 & 8,632 & 8,476 & 9,075 & 8,832 & 9,317 & 9,206 & 9,563 & 10,259 & 10,683 & 11,149 & 11,570 \\
\hline Foreign currency deposits & 2,311 & 2,170 & 2,429 & 2,233 & 2,494 & 2,392 & 2,486 & 2,667 & 2,724 & 2,921 & 3,031 \\
\hline M2 & 6,321 & 6,306 & 6,646 & 6,599 & 6,823 & 6,814 & 7,076 & 7,592 & 7,959 & 8,228 & 8,538 \\
\hline Currency in circulation & 1,649 & 1,519 & 1,734 & 1,567 & 1,750 & 1,561 & 1,628 & 1,731 & 1,815 & 1,769 & 1,887 \\
\hline Deposits (Tanzania Sh) & 4,672 & 4,787 & 4,912 & 5,031 & 5,073 & 5,252 & 5,449 & 5,861 & 6,144 & 6,459 & 6,651 \\
\hline \multicolumn{12}{|l|}{ Memorandum items: } \\
\hline M3 growth (12-month percent change) & 21.7 & 19.5 & 21.7 & 18.4 & 21.1 & 20.6 & 21.6 & 21.0 & 21.0 & 21.1 & 21.0 \\
\hline Foreign currency deposits (12-month percent change) & 26.1 & 18.5 & 21.7 & 11.9 & 21.1 & 14.7 & 18.5 & 22.9 & 22.0 & 22.1 & 21.9 \\
\hline M2 growth (12-month percent change) & 20.1 & 19.9 & 21.7 & 20.8 & 21.1 & 22.8 & 22.7 & 20.4 & 20.6 & 20.8 & 20.7 \\
\hline Currency in circulation (12-month percent change) & 13.7 & 4.8 & 20.5 & 8.9 & & 11.9 & 14.3 & 15.8 & 15.8 & 15.7 & 15.9 \\
\hline Credit to nongovernment sector (12-month percent change) & 39.6 & 26.2 & 26.7 & 9.4 & 26.2 & 10.1 & 14.5 & 18.6 & 21.3 & 21.4 & 21.3 \\
\hline Average reserve money (12-month percent change) & 20.6 & 21.1 & 28.5 & 26.7 & 22.3 & 21.2 & 21.5 & 18.5 & 18.5 & 18.5 & 18.5 \\
\hline Reserve money multiplier (M3/average reserve money) & 3.0 & 3.0 & 3.0 & 3.0 & 3.0 & 3.0 & 3.0 & 3.0 & 3.0 & 3.0 & 3.0 \\
\hline Velocity of money (M3; average) & $\ldots$ & $\ldots$ & $\ldots$ & $\ldots$ & $\ldots$ & $\ldots$ & 3.3 & $\ldots$ & $\ldots$ & $\ldots$ & 3.3 \\
\hline Nonbank financing of the government (net) ${ }^{1}$ & 10 & -66 & 20 & -2 & 30 & 30 & 40 & 0 & 10 & 10 & 40 \\
\hline Bank financing of the government (net) ${ }^{1}$ & 269 & -141 & 72 & 14 & 135 & 138 & 466 & 250 & 201 & 221 & 310 \\
\hline Bank and nonbank financing of the government (net) ${ }^{1}$ & 279 & -208 & 92 & 12 & 165 & 168 & 506 & 250 & 211 & 231 & 350 \\
\hline
\end{tabular}

Sources: Bank of Tanzania; and Fund staff estimates and projections.

${ }^{1}$ Cumulative from the beginning of the fiscal year (July 1). 
Table 6. Tanzania: Balance of Payments, 2007/08-2012/13 (Millions of U.S. dollars, unless otherwise indicated)

\begin{tabular}{|c|c|c|c|c|c|c|}
\hline & $2007 / 08$ & $2008 / 09$ & $\begin{array}{l}\text { 2009/10 } \\
\text { Proj. }\end{array}$ & $\begin{array}{r}\text { 2010/11 } \\
\text { Proj. }\end{array}$ & $\begin{array}{r}2011 / 12 \\
\text { Proj. }\end{array}$ & $\begin{array}{r}2012 / 13 \\
\text { Proj. }\end{array}$ \\
\hline Current account & $-2,012$ & $-2,102$ & $-1,950$ & $-2,383$ & $-2,616$ & $-2,748$ \\
\hline Trade balance & $-3,070$ & $-3,001$ & $-2,933$ & $-3,171$ & $-3,371$ & $-3,480$ \\
\hline $\begin{array}{l}\text { Exports, f.o.b. } \\
\text { of which: }\end{array}$ & 2,609 & 2,969 & 3,216 & 3,826 & 4,168 & 4,565 \\
\hline Traditional agricultural products & 337 & 488 & 441 & 506 & 560 & 629 \\
\hline Gold & 920 & 825 & 1,124 & 1,301 & 1,349 & 1,416 \\
\hline Imports, f.o.b & $-5,667$ & $-5,970$ & $-6,149$ & $-6,996$ & $-7,539$ & $-8,045$ \\
\hline of which: Oil & $-1,683$ & $-1,433$ & $-1,471$ & $-1,797$ & $-1,958$ & $-2,104$ \\
\hline Services (net) & 401 & 232 & 277 & 273 & 284 & 303 \\
\hline of which: Travel receipts & 1,186 & 1,202 & 1,322 & 1,454 & 1,600 & 1,792 \\
\hline Income (net) & -28 & -65 & -71 & -132 & -191 & -249 \\
\hline of which: interest on central government debt & -20 & -27 & -27 & -37 & -103 & -148 \\
\hline Current transfers (net) & 686 & 732 & 778 & 647 & 662 & 677 \\
\hline of which: program grants & 663 & 703 & 745 & 612 & 624 & 636 \\
\hline Capital account & 627 & 428 & 706 & 683 & 672 & 682 \\
\hline of which: project grants & 570 & 366 & 640 & 653 & 660 & 670 \\
\hline Financial account & 1,491 & 1,515 & 1,591 & 1,994 & 2,274 & 2,345 \\
\hline Foreign Direct Investment & 696 & 591 & 510 & 700 & 805 & 916 \\
\hline $\begin{array}{l}\text { Public Sector, net } \\
\text { of which: }\end{array}$ & 840 & 768 & 962 & 1,237 & 1,210 & 1,102 \\
\hline Program loans & 458 & 400 & 670 & 443 & 419 & 428 \\
\hline Non-concessional borrowing & & & & 525 & 525 & 451 \\
\hline Project loans & 419 & 387 & 325 & 331 & 338 & 345 \\
\hline Scheduled amortization ${ }^{1}$ & -38 & -19 & -33 & -63 & -73 & -121 \\
\hline Commercial Banks' NFA (increase $=-$ ) & 157 & 165 & -140 & -60 & 61 & 71 \\
\hline SDR allocation ${ }^{2}$ & $\ldots$ & $\ldots$ & 249 & $\ldots$ & $\ldots$ & $\ldots$ \\
\hline Other private inflows & & -9 & 10 & 117 & 197 & 256 \\
\hline Errors and omissions & 126 & -17 & 0 & 0 & 0 & 0 \\
\hline Overall balance & 232 & -176 & 347 & 295 & 330 & 280 \\
\hline Financing & -232 & 176 & -347 & -295 & -330 & -280 \\
\hline Change in BoT reserve assets (increase, -) & -232 & -69 & -441 & -295 & -330 & -280 \\
\hline Use of Fund credit & 0 & 246 & 94 & 0 & 0 & 0 \\
\hline Fiscal financing gap & & & 0 & 0 & 0 & 0 \\
\hline \multicolumn{7}{|l|}{ Memorandum items: } \\
\hline Gross official reserves (BoT) & 2,660 & 2,925 & 3,461 & 3,755 & 4,085 & 4,365 \\
\hline Months of imports of goods and services (current year) & 4.5 & 4.6 & 5.2 & 5.0 & 5.0 & 5.0 \\
\hline \multicolumn{7}{|l|}{ Current account deficit (percent of GDP) } \\
\hline Including official current transfers & -10.6 & -9.8 & -8.3 & -9.3 & -9.4 & -9.0 \\
\hline Foreign program and project assistance (percent of GDP) & 11.1 & 8.6 & 10.1 & 8.0 & 7.3 & 6.8 \\
\hline Nominal GDP & 19,017 & 21,459 & 23,510 & 25,526 & 27,794 & 30,451 \\
\hline
\end{tabular}

Sources: Tanzanian authorities; and Fund staff estimates and projections.

${ }^{1}$ Relief on some projected external debt obligations is being negotiated with a number of creditors.

2 Tanzania received SDR 147.4 million on August 28 and SDR 11.7 million on September 9 (equivalent to US $\$ 249$ million in total). 
Table 7.Tanzania: Selected Financial Soundness Indicators, 2006-09

\begin{tabular}{|c|c|c|c|c|c|c|c|}
\hline & 2006 & 2007 & 2008 & Mar-09 & Jun-09 & Sep-09 & Dec-09 \\
\hline \multicolumn{8}{|l|}{ Capital adequacy } \\
\hline Regulatory Tier I capital to risk-weighted assets & 15.5 & 15.8 & 14.5 & 20.5 & 18.4 & 19.3 & 18.1 \\
\hline Regulatory Tier I+II capital to risk-weighted assets & 16.3 & 16.2 & 14.9 & 21.2 & 18.8 & 19.7 & 18.5 \\
\hline Regulatory capital to total assets & 7.6 & 8.1 & 8.8 & 12.2 & 10.6 & 11.0 & 11.1 \\
\hline \multicolumn{8}{|l|}{ Asset quality } \\
\hline Nonperforming loans (net of provisions) to gross loans & 6.8 & 6.3 & 6.2 & 7.4 & 7.8 & 6.4 & 6.7 \\
\hline $\begin{array}{l}\text { Nonperforming loans (net of provisions) to total capital } \\
\text { Sectoral distribution of loans }\end{array}$ & 21.1 & 22.0 & 25.2 & 22.4 & 25.8 & 17.8 & 17.3 \\
\hline Agriculture & 9.0 & 11.9 & 10.4 & 10.6 & 11.1 & 12.3 & 10.4 \\
\hline Personal loans & $\ldots$ & $\ldots$ & $\ldots$ & 13.8 & 13.7 & 11.3 & 12.1 \\
\hline Mining and manufacturing & 18.3 & 20.3 & 14.7 & 21.5 & 20.9 & 20.4 & 21.7 \\
\hline Tourism, hotels, restaurants & $\ldots$ & $\ldots$ & $\ldots$ & 4.6 & 4.8 & 4.4 & 4.4 \\
\hline Trade & 15.2 & 17.0 & 18.5 & 17.5 & 17.8 & 18.5 & 19.0 \\
\hline Transport and communication & 7.9 & 6.9 & 7.2 & 7.5 & 10.1 & 9.7 & 9.3 \\
\hline Construction and property & 5.0 & 5.1 & 4.9 & 4.8 & 4.6 & 5.1 & 5.1 \\
\hline Other & 44.6 & 38.8 & 44.3 & 19.6 & 17.1 & 18.3 & 18.1 \\
\hline \multicolumn{8}{|l|}{ Earnings and profitability } \\
\hline Return on assets & 3.9 & 4.7 & 3.8 & 4.0 & 3.6 & 3.4 & 3.2 \\
\hline Return on equity & 26.7 & 29.0 & 23.2 & 24.2 & 21.2 & 20.7 & 18.4 \\
\hline Net interest income to gross income & 53.2 & 53.8 & 55.0 & 69.9 & 52.3 & 51.4 & 51.4 \\
\hline Non interest expenses to gross income & 43.5 & 42.5 & 48.8 & 44.6 & 45.3 & 46.6 & 47.6 \\
\hline Personnel expenses to noninterest expenses & 39.5 & 40.5 & 40.6 & 44.6 & 45.2 & 43.7 & 42.6 \\
\hline Trading and fee income to total income & 27.1 & 25.7 & 26.0 & 29.9 & 26.5 & 29.1 & 29.6 \\
\hline \multicolumn{8}{|l|}{ Liquidity } \\
\hline Liquid assets / total assets & 48.6 & 48.0 & 37.6 & 36.6 & 37.8 & 41.1 & 40.6 \\
\hline Liquid assets to short term liabilities & 54.2 & 53.0 & 41.7 & 43.7 & 45.2 & 48.5 & 46.5 \\
\hline Total (non-bank) loans to customer deposits & 50.0 & 57.8 & 68.5 & 66.1 & 64.8 & 60.7 & 60.8 \\
\hline \multicolumn{8}{|l|}{ Sensitivity to market risk } \\
\hline Net open position in foreign exchange to capital & -49.0 & -70.5 & -39.9 & -14.0 & -5.9 & -18.8 & -11.5 \\
\hline
\end{tabular}

Source: BOT. 


\section{APPENDIX I. LETTER OF INTENT}

May 10, 2010

Mr. Dominique Strauss-Kahn

Managing Director

International Monetary Fund

Washington, D.C. 20431

U.S.A.

Dear Mr. Strauss-Kahn:

1. Tanzania has continued to benefit from policy advice and financial support from the Fund - under the Policy Support Instrument (PSI), as well as balance of payment support - through the Exogenous Shock Facility (ESF). The implementation of programme under both the PSI and ESF has progressed well with sound macroeconomic policies that have helped to mitigate negative impacts of the global financial and economic crisis. The government intends to preserve macroeconomic stability, consolidate attained achievements and implement strategies for stimulating growth and poverty reduction.

2. During July-December 2009 period, performance under the programme was good. All PSI/ESF programme assessment/performance criteria for December 2009 were attained with good margins. Average reserve money was below the target by Tshs 73.0 billion, while Net International Reserves (NIR) was above the target by USD 575 million. In addition, the narrowing of the current account deficit on account of a decline in import bill coupled with an increase in exports contributed positively to the improvement of NIR. Net domestic financing of the Government budget was below the ceiling by Tshs 83.1 billion.

3. The impact of the global financial crisis on economic growth became more noticeable in 2009 compared to 2008. However, economic indicators suggest that growth was affected by the crisis by a lesser extent than expected. The economy continued to experience inflationary pressures mainly due to high food prices whereas nominal exchange rate remained fairly stable during the period. The Lombard and discount rates were revised downwards to make the instruments more attractive and enhance flexibility in provision of liquidity.

4. Provisional growth indicators depict a slowdown in real GDP growth to 5.4 percent in the first half of 2009, compared to 7.0 percent in the corresponding period in 2008 . The economy is expected to strengthen in the second half and push the real GDP growth to 5.5 percent or higher for 2009 compared to 5.0 percent which was projected earlier. As the world economy recovers from the crisis and the demand for exports improves, the growth is expected to pick up in the medium term.

5. Government policy for the remainder of $2009 / 10$ will continue to focus on appropriate fiscal and monetary policies to stimulate economic activities against the backdrop of the impact of the global economic crisis. In the medium term, the government will put emphasis on the priority areas that will accelerate pro-poor economic growth in line with MKUKUTA II. 
6. Following discussions and consultations with the Fund staff, I hereby transmit the letter of intent and memorandum of economic and financial policies which reviews the implementation of the programme during the first half of 2009/10 and describes the objectives and policies that the government intends to pursue during the remainder of the fiscal year and 2010/11 and over the medium term.

7. In view of this performance and in support of our policies going forward, the Government of Tanzania requests the completion of the seventh review under the PSI and the second review under the ESF arrangement, the disbursement of the third tranche under the ESF arrangement, equivalent to SDR 19.89 million, and a new three-year programme under the PSI framework.

8. The Government of Tanzania is confident that the policies set forth in the attached MEFP are adequate to achieve the objectives of its programme but it will, in further consultation with the Fund, take any appropriate measures for this purpose. We will regularly update the Fund on developments in our economic and financial policies, and provide the data needed for the monitoring of the programme - including in the context of twice-yearly reviews. The first and second reviews under the new PSI are expected to take place by end-November 2010 and end-May 2011, respectively, on the basis of the assessment criteria and structural benchmarks indicated in Tables 1, 2, and 3 attached to this letter. In addition, the Government will consult regularly with the Fund on any relevant developments at the initiative of the Government or the Fund.

9. The Government of Tanzania intends to disseminate this letter and the attached MEFP as well as related Fund staff reports, and hereby authorizes the IMF to publish the same on its website after consideration by the Executive Board.

\author{
Yours Sincerely, \\ $/ \mathrm{s} /$ \\ Mustafa H Mkulo (MP) \\ MINISTER FOR FINANCE AND ECONOMIC AFFAIRS \\ UNITED REPUBLIC OF TANZANIA
}

Attachment: Memorandum of Economic and Financial Policies. 


\section{Attachment I. Tanzania: Memorandum of Economic and Financial Policies}

\section{RECENT MACROECONOMIC DEVELOPMENTS AND POLICIES FOR THE REMAINDER OF 2009/10}

\section{Recent economic developments}

1. Impact of the global financial crisis (GFC) on economic growth became more noticeable in 2009 compared to 2008, albeit to a lesser extent than expected. This is reflected in the relatively good performance in traditional exports, cement production and the index of manufacturing industry. Provisional figures indicate a slowdown in real GDP growth to 5.4 percent during the first half of 2009 , compared with a growth of 7.0 percent in the corresponding period in 2008. The economy is expected to strengthen in the second half and push the real GDP growth to 5.5 percent or higher for 2009 compared to 5.0 percent which was projected earlier. This is based on the preliminary results from the third quarter of 2009 which indicated strong performance in manufacturing, construction, trade and transport and communication.

2. The economy continued to experience inflationary pressures attributed to food supply shortages in the neighbouring countries and some parts of Tanzania, as well as high domestic transportation costs. Food inflation remained above 17.0 percent up to November 2009, before declining to 14.5 percent in December 2009 and further to 10.1 percent in February 2010 giving an indication of improving food supply in the region. Non-food inflation rate stayed below 5.0 percent up to October 2009, before bouncing back to 8.4 percent and 10.0 percent in December 2009 and January 2010, respectively, due to rebound in the world oil prices. Recent improvement in food supply in the region led to further decline in headline inflation to 9.6 percent in February 2010.

3. The trade account in 2009 depicted positive developments as low global demand for exports was offset primarily by a reduction in the import bill due to lower import prices. Notwithstanding the low demand for exports, as reflected in the decline in average prices of traditional exports, the value of exports in 2009 increased due to an increase in the volume of all traditional exports, except for tea and tobacco. Gold exports registered large volumes and price increases. Import of goods and services declined during the same period on account of decrease in value of oil import associated with the fall in world market prices. Improved performance of the trade account led to a reduction in current account deficit to USD 978.7 million in the first half of 2009/10, from a deficit of USD 1,214.2 million recorded in the corresponding period a year earlier. During 2009/10, the current account is projected to record a deficit (excluding current transfers) equivalent to 11.5 percent of GDP compared to 13.1 percent of GDP in 2008/09. The overall balance of payments is projected to record a surplus of USD 347.4 million, largely due to improvement in the current account, together with disbursements from development partners and SDR allocation. Consequently, official gross reserves by end-June 2010 are estimated at USD 3,461.0 million, which is equivalent to 5.2 months of imports of goods and services. 


\section{Performance under the programme}

4. All PSI/ESF programme assessment/performance criteria for December 2009 were attained with good margins. Average reserve money was below the target by Tshs 73.0 billion, while Net International Reserves (NIR) was above the target by USD 575 million. The over performance in NIR is largely explained by the narrowing of the current account deficit and the allocation of Special Drawing Rights (SDR). Net domestic financing of the Government budget was below the ceiling by Tshs 83.1 billion.

\section{Fiscal policy}

5. Total revenue collection for the first three quarters of 2009/10 was below budget estimates, with all categories falling short of targets. Total domestic revenue collection (excluding LGAs own sources) was 91 percent of budget estimates during the period. The underperformance was particularly high in excise (87 percent) and non-tax revenue categories ( 80 percent). The under-performance in excise duties is attributed to the nonimplementation of the removal of special fuel levy exemptions granted to existing mining companies. This was prompted by the need to honour existing contracts. In addition, production of the main excisable items, more specifically beer and cigarettes, was below projected levels. The VAT registered a relatively better performance ( 96 percent) despite delayed implementation of an electronic tax registers scheme. Undercollection in non-tax revenues is attributed to a delay in the implementation of new rates, approved in the 2009/10 budget, associated with residence permits, VISAs, and immigration fees. In addition, the global economic slowdown coupled with power outages during August and September 2009 impacted adversely on the revenue collection. The shortfall in revenues has been addressed through frontloading of foreign assistance and modest adjustment of non-priority expenditure.

6. During the first three quarters of 2009/10, expenditure policy focused on mitigating the impact of the global economic crisis, and incorporated unanticipated spending needs due to natural disasters. Government expenditure during the period was Tshs 6,143.9 billion, equivalent to 91.4 percent of the estimated amount. Foreign-financed development expenditure was executed as budgeted while domestically-financed development expenditure was below the budget level by 25 percent. However, enhanced revenue efforts during the fourth quarter should ensure a smooth implementation of planned development projects. Overall recurrent expenditure was below the budget level, despite unanticipated spending related to food distribution to drought affected regions, electricity generation for emergency power supply due to reduced production of hydro-power supply following prolonged droughts in some regions, and teachers' verified claims. In addition, there were continued efforts to repair infrastructure networks and houses damaged by severe flooding in some parts of the country.

7. Budget support for the July 2009-March 2010 period was Tshs 989.6 billion, equivalent to 82.9 percent of the annual budget projection. Net domestic financing recorded a borrowing of Tshs 155.3 billion, well within the ceiling for the period. During the same 
period, grant and loan disbursements for development projects, including basket funds, were 104 percent of the projected level.

8. Revenue collection for $2009 / 10$ is estimated at 94.2 percent of the budget, aided by the expected stronger economic performance in the fourth quarter, as well as additional administrative efforts. The remaining shortfall in domestic revenues and foreign financing will call for some corrective measures during the last three months of the year, including scaling down non-core expenditures without jeopardising service delivery.

\section{Fiscal stimulus}

9. The main thrust of government policy during 2009/10 focused on the combined impact of fiscal and monetary policies in mitigating the severity of the global economic downturn on domestic economic activities, and setting the economy on a firm footing for returning to its medium-term growth path, as the global economic and financial conditions normalize. In this regard, the government put in place an economic rescue plan, totalling about Tshs 1.7 trillion aimed at protecting employment and income, ensuring food security and protection of investment in infrastructure and key social services. The plan includes measures targeted for the most affected sectors, as well as additional financing sources to maintain uninterrupted general expenditures. During the first nine months, 69.6 percent of the plan was implemented: this consisted of Tshs 660.8 billion in domestic financing to fill revenue shortfalls in 2008/09 and 2009/10; Tshs 93.2 billion for food security, and protection of employment and income in adversely affected sectors; Tshs 8 billion for enhancement of existing SMEs and Export Credit Guarantee Schemes; and Tshs 10.0 billion for power generation. Tshs 411.2 billion was received from the IMF through ESF to bolster the level of international reserves. A transparent guideline and monitoring framework for the new guarantee schemes has been put in place in addition to the existing framework for monitoring the SME and export credit guarantee facilities.

\section{Public Financial Management}

10. The Government continues to strengthen public financial management in order to ensure that public resources are utilized in the most efficient and effective manner. This requires effective budget planning, implementation and monitoring in a streamlined system of resource management. After introduction of a new economic classification (GFS 2001) annual accounts are now being prepared using the IPSAS format, which facilitates improved expenditure tracking and monitoring. A functional classification of budget items is being developed, which will also serve as a basis for developing an index for monitoring priority social spending. The internal audit process will be strengthened through the introduction of a centralized independent internal audit department; the necessary amendments to the Public Finance Act, 2004 (Cap 348) were submitted to Parliament in April 2010. The annual report of the Controller and Auditor General for FY2008/09 reveal a sharp upward trend (to 91 percent from 71 the previous year) in unqualified opinions (passing audits) for the central government, while financing reporting of local governments was reported to be considerably improved, partly due to extensive training. 
11. The Cash Management Unit (CMU) continues to prepare a three month rolling cash flow forecast during the year, based on Ministries, Departments and Agencies' (MDA) forecasts. The cash flow forecasts are updated monthly to capture recent developments and are provided to the Budget Ceiling Committee, which allocates resources to MDAs on monthly basis. Efforts have been made to enhance the capacity of the CMU, line Ministries and other spending agencies in order to improve in-year cash flow projection. This will facilitate smooth liquidity forecasting and monetary operations by the Bank.

\section{Monetary and Exchange Rate Policies}

12. During the first eight months of 2009/10, the Bank continued to implement a modestly relaxed monetary policy stance which was adopted in the last quarter of 2008/09. Beginning July 2009, the Bank reviewed the Lombard and discount rates with a view to making them more active instruments of monetary policy and to enhancing flexibility in provision of liquidity to the economy. In spite of this, the window remained inactive as banks continued to maintain a cautious stance in lending to the private sector following the GFC, which resulted to higher liquidity in the banking system. In the first eight months of 2009/10, stock of credit to the private sector grew by 5.0 percent compared with 29.7 percent recorded in the corresponding period a year earlier. Banks increased their preference for Treasury Bills and holdings of net foreign assets: the market for Treasury Bills was oversubscribed by 77.8 percent during July 2009 to February 2010 compared with 2.6 percent recorded in a similar period a year earlier. Change in NFA more than doubled over the same period by Tshs 263.8 billion compared with Tshs 91.1 billion registered in a similar period a year earlier.

13. In the Interbank Foreign Exchange Market (IFEM), the exchange rate continued to be market determined, while the Bank continued to participate primarily to meet liquidity management objectives, while fostering orderly market developments. During the first eight months of 2009/10, the Bank maintained a steady sale of foreign exchange in the IFEM, selling a total of USD 721.0 million, which is more than the USD 640.9 million sold in the corresponding period of 2008/09. Over the period the shilling remained fairly stable, depreciating by only 1.9 percent compared with the depreciation rate of 11.3 percent recorded in a similar period a year earlier.

14. The Bank continued with daily surveillance of the banking system to ensure financial stability and adherence to laws and regulations which is crucial in attaining the broader macroeconomic objectives of the government. As a result of the low level of integration to the global financial system, Bank's effective supervision and surveillance and the impact of the government stimulus package, the financial system in Tanzania remained stable and sound despite the uncertainties associated with the global financial crisis.

15. The Bank will continue to work toward its objective of maintaining macroeconomic stability with low and stable inflation. Given the growth performance in the first three quarters of 2009/10 and gradual recovery in the global economy, monetary policy will remain modestly accommodative to help in stimulating private sector recovery during 2009/10. In this context, the Bank will remain vigilant towards maintaining appropriate 
levels of liquidity that will provide sufficient room for the growth of domestic credit to support macroeconomic recovery. With prudent fiscal policy, favourable weather conditions, and improved food supply in neighbouring countries, it is expected that the targeted inflation rate for end June 2010 of 8 percent will be achieved.

\section{PROGRAMME FOR 2010/11 AND IN THE MEDIUM TERM}

16. In the medium term, the government will continue to pursue prudent macroeconomic policies with the aim of stimulating economic activity. The inflation rate is expected to slow down further in the medium term as food supply improves, the exchange rate remains stable, and fiscal and monetary policies remain prudent. In addition, with the expected recovery in global economic outlook, real GDP growth is projected at 6.2 percent in 2010, and back to pre-crisis levels of around 7.5 percent by 2013. On the other hand, the projected growth in domestic revenue will not be commensurate with needs to finance large-scale infrastructure development projects. To this end, the fiscal deficit after grants is expected to be around 4.8 percent of GDP in the medium term, and will be financed through domestic borrowing, concessional and non-concessional external borrowing.

\section{MKUKUTA}

17. The Government emphasis for the next five years will continue to be in line with the National Development Vision 2025 through a successor poverty reduction strategy (MKUKUTA II). The vision for Tanzania is to become a middle income country, attaining high-quality of life, peace, security and unity, good governance and rule of law, an educated society, and a strong and competitive economy. The current MKUKUTA has registered impressive achievements in most of its social well-being targets. Main achievements include an increase in the enrolment rate at all levels of education, an increase in the number of healthcare facilities, and more women in political and decision making positions. MKUKUTA II has a sharper prioritization on measures to accelerate growth as a sustainable means of reducing poverty. A draft of the successor strategy has been shared with wide range of stakeholders so as to identify gaps, ascertain priorities and generally enhance national ownership of development initiatives. The draft strategy will go through the government approval process by end-June 2010 .

18. MKUKUTA II will place greater emphasis on building up infrastructure, a necessary condition for achieving sustained higher growth. Infrastructure spending will be focused on transportation, power, and communication. It will also place emphasis on growth-enhancing sectoral interventions, for example, agriculture, manufacturing, and tourism, which should have a strong impact on productivity and job creation. Combining infrastructure building and sectoral strategies is expected to create growth synergies, especially for transforming agriculture.

19. The Government's strategy for agricultural development is titled "Kilimo Kwanza" or "agriculture first." The emphasis on the Kilimo Kwanza strategy is intended to boost agricultural productivity and improve food distribution through investment in rural roads and markets. The strategy also aims at improving irrigation infrastructure and access to financing, 
including through the establishment of a commercially-run Agricultural Bank, community banks and enhancement of the window for agricultural credit at Tanzania Investment Bank.

20. The government is committed to support private sector growth. In addition to investment to address infrastructure gaps, a comprehensive programme is being formulated with the overall objective of reducing the cost of doing business in Tanzania. The President appointed a Regulatory Reform Task Team under the leadership of the Prime Minister's Office. The team has come up with a set of recommendations aimed at improving the business environment. These include recommendations on starting business, on dealing with construction permits, on registering property, on employing workers, on trading across borders, on paying taxes, and on getting credit. It is expected that critical short-term measures that involve legislative and statutory reviews and reforms will be submitted to the Parliament in July 2010.

21. The National Social Protection Framework (NSPF) is being developed, which identifies measures to protect vulnerable groups such as: orphans and children; people with disabilities; the elderly; people living with HIV \& AIDS and long term illnesses; vulnerable women; former inmates and people disabled by accidents, wars and conflicts. The NSPF is expected to be approved by the Cabinet by end-June 2010 and the specific interventions are being discussed in the context of the MKUKUTA II.

\section{Fiscal and Monetary Policies}

22. The Government will continue to strengthen domestic resource mobilization in the medium term. Areas under consideration include a widening of the tax base, including the informal sector which will be facilitated by the introduction of national IDs and administrative improvement of the fiscal regime, including management of tax exemptions. The revenue to GDP ratio is projected to increase to 16.3 percent in 2010/11 and further to 16.9 percent by $2012 / 13$.

23. Starting in FY10/11, expenditure policy will place more emphasis on infrastructure investment, while maintaining priority social spending, through gradual withdrawal of fiscal stimulus measures and rationalizing non-priority goods and services spending. Total expenditure is projected be 27.1 percent of GDP in 2010/11, and decline to 25.8 percent of GDP in 2012/13. The spending level in the medium term reflects conservative assumptions on donor financing, as well as increased revenue collection and non-concessional borrowing.

24. The Government will continue to finance development projects mainly through concessional borrowing and will retain its own 35 percent grant element floor for external borrowing. However, in light of the pressing infrastructure needs, the government intends to make use of additional domestic financing of 1 percent of GDP each year and nonconcessional external financing of up to USD 1.5 billion over the 3 year PSI period, roughly evenly phased. Priority projects have already been identified in line with the Medium-term Public Investment Plan (MPIP, see paragraph 29), including roads and railways that are critical for improving the integration of transportation networks within the region and increased capacity of energy generation. While the government will seek to maximize the concessionality of new borrowing, necessary exemptions to the grant element floor specified 
in the Government Loans, Guarantees and Grants Act 1974 (As amended in 2004) will be approved for specific projects with a clear high rate of return and with due consideration for absorptive capacity constraints. The government has resumed the process of identifying firms which will provide consultancy services on sovereign credit rating and subsequent issuance of a Eurobond. The anticipated level of borrowing would not affect the sustainability of debt as indicated by the 2009 World Bank/IMF Debt Sustainability Analysis. The Government will continue to monitor closely public debt developments, and is formulating a comprehensive Medium Term Debt Strategy which will be completed in October 2010. The medium term debt strategy will be revisited every two years and will include a detailed analysis of contingent liabilities, including those arising from government guarantees.

25. The Government will continue reforms in public financial management to ensure effective planning, implementation of public spending and integrity of the budget. The strengthening of the alignment of annual budget with the medium term plan will be given continued priority. To this end, MoFEA will improve outer year revenue projections to provide execution bodies with more concrete ceilings, which would provide a realistic signal about the actual resource envelope. Outer year spending plans will also be strengthened to make them built on medium term affordability consideration with specific projects clearly prioritised. To ensure timely execution of budget expenditures, the procurement law will also be reviewed with the objective of streamlining the procurement process.

26. Commitment control and expenditure tracking and monitoring will continue to be strengthened. MoFEA has already issued a guideline to prohibit expenditure commitment beyond the budget, and will continue to monitor expenditure development at spending agencies in a timely manner. As a means for improving monitoring of the process for clearing payment claims, MoFEA will begin collecting data on a quarterly basis beginning with end-June 2009 (to be reported by end-September 2009), in three of the largest spending ministries (Infrastructure, Health, Home Affairs), on the amount of payment claims outstanding more than 30 days, 60 days and 90 days. Monitoring the stock of claims outstanding over time will enable a more accurate monitoring of the fiscal situation, and facilitate better projections of cash flow needs and smoother implementation of monetary policy.

27. A bridge table for GFS functional classification has been developed to facilitate an effective monitoring of the alignment between policy objectives and actual expenditure, and the 2010/11 budget will include a presentation consistent with GFS2001 functional classification.

28. The Government will continue to implement the Medium-Term Pay Policy (MTPP) focussing mainly on recruiting new staff in education, agriculture, livestock, irrigation and health sectors, as well as maintaining competitive salary levels, transparency and equity in payscales for the public service. The medium-term projection indicates that wages will be around 6.1 percent of GDP in 2010/11 to 2012/13.

29. The government has prepared the Medium-term Public Investment Plan (MPIP) with the aim of improving the alignment between policy objectives and the budget by providing 
enhanced policy coordination and clearer prioritization of public investment projects. The MPIP has identified priority infrastructure projects that will accelerate economic growth and poverty reduction, such as energy, agriculture and transportation. Given budgetary resource constraints and potential efficiency gains, greater participation of the private sector in financing and/or implementing infrastructure projects through Public Private Partnership (PPP) arrangements will be encouraged. In this regard, the Government is working on legal and institutional frameworks for PPPs, and plans are underway to establish a PPP Unit in the MoFEA to manage associated fiscal risks and provide advisory services for promotion and contracting of PPPs.

30. Monetary policy will focus on maintaining sufficient liquidity among banks to support credit growth, while preserving the primary objective of attaining and maintaining low and stable inflation. During financial year 2010/11, the Bank is targeting annual growth rate of average reserve money of 18.5 percent and broader monetary aggregates, M2 and M3 at 20.7 percent and 21.0 percent, respectively, which should be consistent with an adequate supply of credit to the economy. The rebound of world oil prices could pose a risk to sustaining lower inflation. Another challenge may come from potential disruptions to the transportation network caused by natural disasters, such as flooding.

31. The Bank will remain vigilant in safeguarding the current stability in the financial sector as the adverse effects of the global financial turmoil subside. The Bank will maintain strict surveillance over all banks operating in Tanzania on daily basis, and the early warning system which has already been put in place will be enhanced to ensure that all financial indicators remain sound.

32. The Bank's liquidity windows including the intra day loan facility (ILF) and its overnight refinancing facility, i.e. the Lombard window will remain open for banks in need of liquidity to square their positions. The Bank will maintain its foreign exchange policy, including its presence in the foreign exchange market and strengthen its oversight in the market. Measures will be taken to sustain orderly developments in the market and ensure safety in payment systems to protect the financial system from possible contagion effects.

\section{Financial sector stability}

33. The financial sector in Tanzania continues to strengthen following the ongoing Second Generation Financial Sector Reform programme. For the banking sector as a whole, financial indicators are broadly sound. Ongoing reforms are expected to enhance stability and access to financial services, including the establishment of a credit reference system. The procurement process for a consultant to assist in the establishment of this credit reference databank is at an advanced stage, and credit reference bureau regulations and licensing guidelines are in the approval process. Regarding improving access to long term development finance, the Government has approved a framework which will allow the Tanzania Investment Bank to operate as a Development Finance Institution with a window for lending to the agricultural sector. With regard to relocation of credit guarantee schemes that are currently operated by the Bank on behalf of Government, a draft report on the future structure and modus operandi of the schemes outside the Bank is under discussion. 
34. Regarding financial supervision of pension funds, the new Social Security (Regulatory Authority) Act became effective $1^{\text {st }}$ November 2008. The Ministry of Labour, Employment, and Youth Development has finalized regulations to implement the law. Amendments to the existing pension fund-specific legislation to bring them into conformity with the new regulatory regime are already in place after being approved by the Minister. The process of recruiting the CEO for the Regulatory Authority and subsequent issuance of investment guidelines for the pension funds, in consultation with the regulator, is expected to be completed by end-June 2010. In the meantime, the BoT will introduce a data collection and reporting system for pension funds.

35. The Bank continued to implement the recommendations of the IMF Safeguards Assessment Mission of August 2009. The Bank is currently evaluating some firms that expressed interest on advising the Directorate of Internal Audit on issues related to risk assessment. The process of engaging a consulting firm to conduct a comprehensive risk assessment at the Bank is at the contract negotiation stage. The 2008/09 statutory audit commenced in October 2009 and the audit report (unqualified opinion) was adopted by the Board on $22^{\text {nd }}$ December 2009. The accounts were published in the Bank website in January 2010.

36. A tentative plan for timing and sequencing of capital account liberalization was included in the EAC Common market protocol which was signed on $20^{\text {th }}$ November 2009 by Heads of States. The Bank management is working on the development of an action plan toward the gradual lifting of restrictions/residual controls as proposed in the timing and sequencing plan. The removal of restrictions shall be progressive in accordance with the schedule on the Free Movement of Capital specified as an annex to the Common Market Protocol. Among the restrictions that the Government intends to eliminate by 2012, include sale or issue of debt securities abroad and outward direct investments by residents.

37. The Bank is preparing an action plan to address key issues identified as part of the FSAP update. Main areas of focus on the action plan will be on safeguarding financial stability, improving financial intermediation, promoting long-term finance and deepening financial access. A new Financial Stability Report, reinforcing macro prudential surveillance, will be issued to the BoT Board by end-June 2010. Meanwhile, the Bank, in collaboration with the Tanzanian Investment Center and the National Bureau of Statistics, continues to perform annual surveys to monitor private capital flows, including foreign direct investment. The Bank will need to develop capacity for high frequency monitoring of portfolio capital flows as a precursor to capital account liberalization.

38. An outline that provides a broad framework for Crisis Management for the whole financial sector was produced in February 2010 in response to FSAP recommendations. A follow up mission to guide the development of crisis management in each component of the financial sector is scheduled for August 2010. The Bank of Tanzania has formulated an action plan to develop the crisis management framework for the banking industry and other regulatory agencies have been informed about the requirement to prepare crisis management 
frameworks in their sectors. The Tanzania Financial Regulators Forum, which is scheduled to be launched in the third quarter of 2010, will coordinate the preparation of the whole financial sector crisis management framework.

39. In order to deepen coverage of the Deposit Insurance Fund, the Minister for Finance and Economic Affairs has approved the maximum insurance coverage from the current level of Tshs 500,000 to Tshs 1,500,000 per depositor per bank effective May, 2010. Legal process to implement the new level is in the final stages. The new level will meet the recommended limits by the International Association of Deposit Insurers (IADI). Meanwhile, the recommendations to increase minimum capital requirements from Tshs 5 billion to Tshs 15 billion for commercial banks and from Tshs 2.5 billion to Tshs 7.5 billion for financial institutions have been approved by the Board of the Bank of Tanzania. The Bank will also establish, by end-March 2011, a framework for providing emergency liquidity assistance, beyond day-to-day liquidity management tools.

\section{Statistical issues}

40. The National Bureau of Statistics (NBS) is finalizing revision of weights of items in the Consumer Basket on the basis of results from Household Budget Survey (HBS). The new weights will start to be employed in calculating inflation from April, 2010. The new inflation formula will reflect the latest consumption pattern based on the HBS analysis results. The NBS, with the support of the Bank, is also developing a core inflation indicator in order to provide a better assessment of underlying inflation excluding supply-side effects, based on a harmonized methodology to be agreed by all EAC countries.

\section{PROGRAMME MONITORING}

Table 1 indicates quantitative indicative targets for the current PSI-supported program, which ends in May 2010. Quantitative assessment criteria for end-June and end-December 2010 and quantitative indicative targets for end-September 2010 and end-March 2011 will monitor programme implementation under the new PSI (Table 2). The government and IMF staff also agreed on the structural benchmarks listed in Table 3. Further detail is contained in the attached Technical Memorandum of Understanding.

\section{MINISTRY OF FINANCE AND ECONOMIC AFFAIRS \\ DAR ES SALAAM, TANZANIA}

May 10, 2010 
Table 1. Tanzania: Quantitative Assessment/Performance Criteria and Indicative Targets Under the Policy Support Instrument and the Exogenous Shocks Facility, September 2009-March 2010

\begin{tabular}{|c|c|c|c|c|c|c|}
\hline & \multicolumn{4}{|c|}{2009} & \multirow{2}{*}{\multicolumn{2}{|c|}{$\begin{array}{c}2010 \\
\text { March } \\
\end{array}$}} \\
\hline & \multicolumn{2}{|c|}{ September } & \multicolumn{2}{|l|}{ December } & & \\
\hline & $\begin{array}{l}\text { Indicative } \\
\text { Targets }\end{array}$ & Actual & $\begin{array}{c}\text { Assessment/ } \\
\text { Performance Criteria } \\
\text { Program }\end{array}$ & Actual & $\begin{array}{l}\text { Program } \\
\text { Indicative } \\
\text { Targets }\end{array}$ & Prelim. \\
\hline & \multicolumn{6}{|c|}{ (Billions of Tanzania Shillings; end of period, unless otherwise indicated) } \\
\hline $\begin{array}{l}\text { Net domestic financing of the government of Tanzania } \\
\text { (cumulative, ceiling) }\end{array}$ & 279 & -208 & $=0$ & 12 & 168 & 168 \\
\hline Accumulation of budgetary arrears (ceiling; indicative target only) & 0 & 0 & 0 & 0 & 0 & {$[0]$} \\
\hline $\begin{array}{l}\text { Average reserve money (upper bound) }{ }^{3} \\
\text { Average reserve money target }{ }^{3} \\
\text { Average reserve money (lower bound) }\end{array}$ & $\begin{array}{l}2,862 \\
2,833 \\
2,805\end{array}$ & 2,846 & $\begin{array}{l}3,009 \\
2,979 \\
2,949\end{array}$ & 2,936 & $\begin{array}{l}3,089 \\
3,058 \\
3,028\end{array}$ & 3,026 \\
\hline Average reserve money (lower bound) ${ }^{3}$ & \multicolumn{6}{|c|}{ (Millions of U.S. dollars; end of period) } \\
\hline Net international reserves of the Bank of Tanzania (floor) ${ }^{4}$ & 2,234 & 3,292 & 2,648 & 3,223 & 2,726 & 3,223 \\
\hline Accumulation of external payments arrears (ceiling) ${ }^{b}$ & 0 & 0 & 0 & 0 & 0 & {$[0]$} \\
\hline $\begin{array}{l}\text { Contracting or guaranteeing of external debt on } \\
\text { nonconcessional terms (ceiling) }\end{array}$ & 0 & 0 & 0 & 0 & 0 & 0 \\
\hline \multicolumn{7}{|l|}{ Memorandum item: } \\
\hline Foreign program assistance (cumulative grants and loans) ${ }^{1}$ & 315 & 686 & 818 & 809 & 1,139 & 1,107 \\
\hline
\end{tabular}

Note: For precise definitions of the aggregates shown and details of the adjustment clauses, see the Technical Memorandum of Understanding (TMU) attached to the Government's letter of November 13, 2009.

${ }^{1}$ Cumulative from the beginning of the fiscal year (July 1 ).

2 To be adjusted upward by up to TSh 250 billion for the U.S. dollar equivalent of a shortfall in foreign program assistance from the amounts shown in the memorandum item.

${ }^{3}$ Assessment criteria and benchmarks apply to upper bound only.

${ }^{4}$ Floors are set US $\$ 200$ million below projected levels. Floor will be adjusted downward for any shortfall in foreign program assistance from the amounts shown in the memorandum item up to the equivalent of TSh 250 billion.

${ }^{5}$ Continuous PC; excludes debt-service payment arrears pending debt-rescheduling arrears. 
Table 2. Tanzania: Quantitative Assessment Criteria and Indicative Targets Under the Policy Support Instrument, June 2010-June 2011

\begin{tabular}{|c|c|c|c|c|c|}
\hline & \multicolumn{3}{|c|}{2010} & \multicolumn{2}{|c|}{2011} \\
\hline & June & September & December & March & June \\
\hline & $\begin{array}{l}\text { Proposed } \\
\text { Assessment } \\
\text { Criteria }\end{array}$ & $\begin{array}{l}\text { Proposed } \\
\text { Indicative } \\
\text { Targets }\end{array}$ & $\begin{array}{l}\text { Proposed } \\
\text { Assessment } \\
\text { Criteria }\end{array}$ & $\begin{array}{l}\text { Proposed } \\
\text { Indicative } \\
\text { Targets }\end{array}$ & $\begin{array}{c}\text { Proposed } \\
\text { Indicative } \\
\text { Targets }\end{array}$ \\
\hline \multirow[b]{2}{*}{$\begin{array}{l}\text { Net domestic financing of the government of Tanzania } \\
\text { (cumulative, ceiling) }\end{array}$} & \multicolumn{5}{|c|}{ (Billions of Tanzania Shillings; end of period, unless otherwise indicated) } \\
\hline & 506 & 250 & 211 & 231 & 350 \\
\hline $\begin{array}{l}\text { Average reserve money (upper bound) } \\
\text { Average reserve money target }{ }^{3} \\
\text { Average reserve money (lower bound) }{ }^{3}\end{array}$ & $\begin{array}{l}3,193 \\
3,161 \\
3,129\end{array}$ & $\begin{array}{l}3,406 \\
3,372 \\
3,338\end{array}$ & $\begin{array}{l}3,514 \\
3,479 \\
3,444\end{array}$ & $\begin{array}{l}3,622 \\
3,586 \\
3,550\end{array}$ & $\begin{array}{l}3,783 \\
3,746 \\
3,708\end{array}$ \\
\hline & \multicolumn{5}{|c|}{ (Millions of U.S. dollars; end of period) } \\
\hline Net international reserves of the Bank of Tanzania (floor) ${ }^{4}$ & 3,079 & 3,061 & 3,207 & 3,317 & 3,374 \\
\hline Accumulation of external payments arrears (ceiling) & 0 & 0 & 0 & 0 & 0 \\
\hline $\begin{array}{l}\text { Contracting or guaranteeing of external debt on } \\
\text { nonconcessional terms (continuous ceiling) }\end{array}$ & 0 & 525 & 525 & 525 & 525 \\
\hline \multicolumn{6}{|l|}{ Memorandum item: } \\
\hline Foreign program assistance (cumulative grants and loans) ${ }^{1}$ & 1,416 & 329 & 770 & 933 & 1,055 \\
\hline
\end{tabular}

Note: For precise definitions of the aggregates shown and details of the adjustment clauses, see the Technical Memorandum of Understanding (TMU) attached to the Government's letter of May10, 2010.

${ }^{1}$ Cumulative from the beginning of the fiscal year (July 1 ).

2 To be adjusted upward by up to TSh 250 billion for the U.S. dollar equivalent of a shortfall in foreign program assistance from the amounts shown in the memorandum item.

${ }^{3}$ Assessment criteria and benchmarks apply to upper bound only.

${ }^{4}$ Floor will be adjusted downward for any shortfall in foreign program assistance from the amounts shown in the memorandum item up to the equivalent of TSh 250 billion.

${ }^{5}$ Continuous assessment criteria; ceiling applies throughout the year. 
Table 3. Proposed Structural Benchmarks During First Year of New PSI, 2010/11

\begin{tabular}{ccc}
\hline Measure & Target Date of \\
Implementation
\end{tabular}

Poverty Reduction Strategy

Adoption of MKUKUTA II by Cabinet.

Approval by Cabinet of a new National

Social Protection Framework.

\section{Financial Sector}

Appoint Head of Social Security Regulatory Agency and issue investment guidelines for pension funds.

Introduce data collection and reporting system for pension funds.

Submit Financial Stability Report to the BoT Board.

Notification to banks to increase contributions to the Deposit Insurance Fund.

Establish framework for emergency liquidity assistance beyond day-to-day liquidity management and tools.

\section{Fiscal}

Debt management: Prepare a Medium-Term Debt Strategy consistent with resource needs under the new PRS and reflecting contingent liabilities.

Establish a PPP unit within the MoFEA.

Develop an index for monitoring priority social spending.

Collect data, on a quarterly basis, on payment claims outstanding over 30,60, and 90 days in Ministries of Infrastructure, Health, and Home Affairs. End-June data to be provided by end-September.

\section{Statistics}

Develop core inflation index.
End-June, 2010

End-June, 2010

End-June, 2010

End-June, 2010

End-June, 2010

End-June, 2010

End-March, 2011

End-December, 2010

Critical for ensuring sustainability of the government's borrowing strategy and for facilitating broader access to capital markets.

End-December, 2010

End-December, 2010

End-June, 2010

End-December, 2010
Provides the overall framework for policy interventions.

To provide a reliable and comprehensive social safety net targeted to the most vulnerable and better automatic stabilizers in the event of future shocks.

Absence of effective regulation/supervision of the social security funds - which hold assets of 10 percent of GDP or one quarter of the financial system - adds considerable risk to the financial system and public finances.

Enables higher frequency and more accurate monitoring of risks in the financial sector.

Reinforces macro prudential surveillance.

Deepens deposit insurance coverage.

Provides mechanism for expedited handling of liquidity problems with systemic implications.
Essential for MoFEA to be able to implement its gate-keeping responsibilities specified in the PPP legislation.

Facilitates monitoring the implementation of social spending.

Enables a more accurate monitoring of the fiscal situation, and facilitates better projections of cash flow needs and smoother implementation of monetary policy.

Provides more meaningful indicator of the stance of monetary policy and a key EAC convergence indicator. 


\section{Attachment II. Technical Memorandum of Understanding on Selected Concepts and Definitions Used in the Monitoring of the PSI-Supported Program}

\section{INTRODUCTION}

1. The purpose of this Technical Memorandum of Understanding (TMU) is to describe concepts and definitions that are being used in the monitoring of the quantitative PSI assessment criteria and indicative targets under Tanzania's program supported by the PSI arrangement. The principal data source is the standardized reporting forms, 1SR and 2SR, as provided by the Bank of Tanzania to the IMF, and the government debt tables provided by the Accountant General's office.

\section{DEFInitions}

\section{Net international reserves}

2. Net international reserves (NIR) of the Bank of Tanzania (BoT) are defined as reserve assets minus reserve liabilities. The BoT's reserve assets, as defined in the IMF BOP manual ( $5^{\text {th }}$ edition) and elaborated in the reserve template of the IMF's special data dissemination standards (SDDS), include: (i) monetary gold; (ii) holdings of SDRs; (iii) the reserve position at the IMF; (iv) all holdings of foreign exchange; and (v) other liquid and marketable assets readily available to the monetary authorities. Reserve assets exclude assets pledged or otherwise encumbered, including but not limited to assets used as collateral or guaranteed for a third party external liability (assets not readily available). The BoT's reserve liabilities include: (i) all short-term foreign exchange liabilities to nonresidents, of original maturities less than one year; and (ii) outstanding purchases and loans from the IMF.

\section{Reserve money and reserve money band}

3. Reserve money is defined as the sum of currency issued by the BoT and the deposits of commercial banks with the BoT. The reserve money targets are the projected daily averages of March, June, September, and December within a symmetrical one percent band. The upper bound of the band serves as the assessment criterion or indicative target.

\section{Net domestic financing of the Government of Tanzania}

4. Net domestic financing of the Government of Tanzania (NDF) includes financing of the budget of the central (union) government of Tanzania ("government") by the banking system (BoT and commercial banks) and the nonbank public. NDF is calculated as the cumulative change since the beginning of the fiscal year in the sum of (i) loans and advances to the government by the BoT and holdings of government securities and promissory notes (including liquidity paper issued by the BoT for monetary policy purposes), minus all government deposits with the BoT; (ii) all BoT accounts receivable due from the Government of Tanzania that are not included under (i) above; (iii) loans and advances to the government by other depository corporations and holdings of government securities and promissory notes, minus all government deposits held with other depository corporations; and (iv) the outstanding stock of domestic debt held outside depository

corporations excluding: government debt issued for the recapitalization of the NMB and TIB; debt swaps with COMELCO (Russia) and the government of Bulgaria; mortgage on acquired sisal 
estates; compensation claims; and debt of parastatal companies assumed by the government. Government deposits that reflect the unspent portion of nonconcessional borrowing designated for specific infrastructure projects, and which are held in ring-fenced accounts exclusively for that purpose, will be excluded from (i) and (iii) above in the calculation of net domestic financing.

\section{Government deposits at the BoT}

5. Government deposits at the BoT include government deposits as reported in the BoT balance sheet, 1SR -including counterpart deposits in the BoT of liquidity paper issued for monetary policy purposes, and foreign currency-denominated government deposits at the BoT, including the PRBS accounts and the foreign currency deposit account.

\section{External payments arrears}

6. External payments arrears consist of the total amount of external debt service obligations (interest and principal) of the government and the BoT that have not been paid at the time they are due, excluding arrears on external debt service obligations pending the conclusion of debtrescheduling arrangements. The ceiling on external payments arrears is continuous and applies throughout the year.

\section{Contracting or guaranteeing of external debt on nonconcessional terms}

7. The term "external debt" will have the meaning set forth in Point 9 of the Guidelines on Performance Criteria with Respect to External Debt. External debt will be determined on the basis of currency of denomination of the debt. Government external debt is outstanding debt owed or guaranteed by the Government of Tanzania or the Bank of Tanzania. The scope of the criterion will be expanded at the time of the first review of the PSI-supported program to include public sector debt, including public enterprises that are not explicitly excluded. External debt contracted in the interim will be subject to the program ceiling according to the revised definition as of the first review; the ceiling on external debt is continuous and applies throughout the year.

8. Government debt is considered nonconcessional if the grant element is lower than 35 percent, calculated using discount rates based on Organization for Economic Cooperation and Development (OECD) commercial interest reference rates (CIRR), adjusted as appropriate for different maturities. For maturities of less than 15 years, the grant element will be calculated based on 6-month averages of commercial interest rates. For maturities longer than 15 years, the grant element will be calculated based on 10-year averages. This PSI assessment criterion applies not only to debt as defined in Point 9 of the Guidelines on Performance Criteria with Respect to External Debt, but also to commitments contracted or guaranteed for which value has not been received.

\section{Foreign program assistance}

9. Foreign program assistance is defined as budget support and basket grants and loans received by the Ministry of Finance and Economic Affairs (MoFEA) through BoT accounts and accounts at other depository corporations and is calculated as the cumulative sum, since the 
beginning of the fiscal year, of the receipts from (i) program loans and (ii) program grants. Program assistance does not include nonconcessional external debt as defined in paragraphs 7 and 8 .

\section{Program exchange rate}

10. For 2010/11, the end-of-period program exchange rates are set at T Sh 1360 per U.S. dollar, T Sh 1370 per U.S. dollar, T Sh 1380 per U.S. dollar, and T Sh 1390 per U.S. dollar for Quarters IIV, respectively. For 2010/11, the period average program exchange rates are T Sh 1355 per U.S. dollar, T Sh 1365 per U.S. dollar, T Sh 1375 per U.S. dollar, and T Sh 1385 per U.S. dollar for Quarters I-IV, respectively. For 2010/11 as a whole, the program average exchange rate is set at T Sh 1370 per U.S. dollar.

\section{ADJUSTERS}

\section{Net international reserves}

11. The end-June, end-September, end-December 2010 and end-March 2011 quantitative targets for the BoT's net international reserves will be adjusted downward by the amount in U.S. dollars of any shortfall in foreign program assistance in U.S. dollars (up to a limit of T Sh 250 billion, converted into U.S. dollars at the corresponding program average exchange rate), relative to projections shown in the Quantitative PSI Assessment Criteria and Indicative Targets Table attached to the applicable Letter of Intent and Memorandum of Economic and Financial Polices of the Government of Tanzania.

\section{Net domestic financing}

12. The end-June, end-September, end-December 2010 and end-March 2011 quantitative limits on the net domestic financing of the Government of Tanzania will be adjusted upward for any shortfall in foreign program assistance in U.S. dollars (up to a limit of T Sh 250 billion, evaluated using the corresponding program annual average exchange rate), relative to projections shown in the Quantitative PSI Assessment Criteria and Indicative Targets Table attached to the applicable Letter of Intent and Memorandum of Economic and Financial Polices of the Government of Tanzania.

\section{DATA Reporting REQUiREMENTS}

13. For purposes of monitoring the program, the Government of Tanzania will provide the data listed in Table 1 below. 
Table 1 Summary of Reporting Requirements

\begin{tabular}{|c|c|c|c|}
\hline Information & $\begin{array}{l}\text { Reporting } \\
\text { Institution }\end{array}$ & Frequency & $\begin{array}{c}\text { Submission } \\
\text { Lag }\end{array}$ \\
\hline Issuance of government securities. & BoT & Bi-weekly & 1 week \\
\hline Yields on government securities. & BoT & Bi-weekly & 1 week \\
\hline Consumer price index. & NBS & Monthly & 2 weeks \\
\hline $\begin{array}{l}\text { The annual national account statistics in } \\
\text { current and constant prices. }\end{array}$ & NBS & Annually & 6 months \\
\hline $\begin{array}{l}\text { The quarterly national account statistics in } \\
\text { constant prices. }\end{array}$ & NBS & Quarterly & 3 months \\
\hline Balance sheet of the BoT (1SR). & BoT & Monthly & 1 week \\
\hline $\begin{array}{l}\text { Consolidated accounts of other depository } \\
\text { corporations and the depository } \\
\text { corporations survey (2SR and the DCS). }\end{array}$ & BoT & Monthly & 4 weeks \\
\hline $\begin{array}{l}\text { Summary of stock of external debt, } \\
\text { external arrears, and committed } \\
\text { undisbursed loan balances by creditor. }\end{array}$ & BoT & Monthly & 2 weeks \\
\hline External trade developments. & BoT & Monthly & 4 weeks \\
\hline Balance of payments & BoT & Quarterly & 4 weeks \\
\hline $\begin{array}{l}\text { Standard off-site bank supervision } \\
\text { indicators for other depository corporations. }\end{array}$ & BoT & Quarterly & 6 weeks \\
\hline $\begin{array}{l}\text { Financial Soundness Indicators for other } \\
\text { depository corporations. }\end{array}$ & BoT & Quarterly & 6 weeks \\
\hline $\begin{array}{l}\text { Other depository corporation lending by } \\
\text { activity. }\end{array}$ & BoT & Monthly & 4 weeks \\
\hline Commercial banks interest rate structure. & BoT & Monthly & 4 weeks \\
\hline $\begin{array}{l}\text { Summary table of: (i) average reserve } \\
\text { money; (ii) net domestic financing of the } \\
\text { government; (iii) stock of external arrears; } \\
\text { (iv) new contracting or guaranteeing of } \\
\text { external debt on nonconcessional terms; } \\
\text { and (v) net international reserves. }{ }^{1}\end{array}$ & BoT and MoFEA & Quarterly & 4 weeks \\
\hline
\end{tabular}

\footnotetext{
${ }^{1}$ The MoFEA and BoT will reconcile data on BoT claims on the government, to ensure that such claims recorded in the BoT balance sheet are the same as those reported by the Accountant General of the MoFEA.
} 


\begin{tabular}{|c|c|c|c|}
\hline Information & $\begin{array}{l}\text { Reporting } \\
\text { Institution }\end{array}$ & Frequency & $\begin{array}{l}\text { Submission } \\
\text { Lag }\end{array}$ \\
\hline $\begin{array}{l}\text { Amount of payment claims outstanding of } \\
\text { more than } 30 \text { days, } 60 \text { days and } 90 \text { days in } \\
\text { the Infrastructure, Health and Home Affairs } \\
\text { ministries. For each ministry, total claims } \\
\text { outstanding to be divided into: (i) unverified } \\
\text { claims; and (ii) claims verified but not yet } \\
\text { paid. }\end{array}$ & MoFEA & Quarterly & 3 months \\
\hline $\begin{array}{l}\text { The flash report on revenues and } \\
\text { expenditures. }\end{array}$ & MoFEA & Monthly & 4 weeks \\
\hline The TRA revenue report & TRA & Monthly & 4 weeks \\
\hline The monthly domestic debt report. ${ }^{1}$ & MoFEA & Monthly & 4 weeks \\
\hline $\begin{array}{l}\text { Monthly report on central government } \\
\text { operations. }\end{array}$ & MoFEA & Monthly & 4 weeks \\
\hline $\begin{array}{l}\text { Detailed central government account of } \\
\text { disbursed budget support grants and loans, } \\
\text { and external debt service due and paid. }\end{array}$ & MoFEA & Monthly & 4 weeks \\
\hline $\begin{array}{l}\text { Detailed central government account of } \\
\text { disbursed donor project support grants and } \\
\text { loans. }\end{array}$ & MoFEA & Monthly & 4 weeks \\
\hline $\begin{array}{l}\text { Statement on new loans contracted during } \\
\text { the period including terms and conditions } \\
\text { according to loan agreements. }\end{array}$ & MoFEA & Quarterly & 4 weeks \\
\hline
\end{tabular}




\title{
INTERNATIONAL DEVELOPMENT ASSOCIATION AND INTERNATIONAL MONETARY FUND ${ }^{1}$
}

\author{
UNITED REPUBLIC OF TANZANIA
}

\author{
Joint World Bank/IMF Debt Sustainability Analysis \\ Prepared by the staffs of the International Development Association and \\ International Monetary Fund \\ Approved by Sudarshan Gooptu and Sudhir Shetty (World Bank) \\ and Saul Lizondo and Dominique Desruelle (IMF)
}

May 18, 2010

Tanzania's risk of debt distress remains low, reflecting large debt relief and sound macroeconomic policies in recent years. In the coming years, the government anticipates borrowing from both domestic and external sources, including on non-concessional terms, to finance a stepping up of infrastructure spending. The additional borrowing will increase the present value (PV) of debt-toGDP ratio but should not jeopardize long-term debt sustainability, although debt service indicators will increase more significantly, due to an assumption of the most expensive terms. In addition, alternative downside scenarios illustrate that debt indicators would be sensitive to significantly lower long-term growth. This highlights the importance of a sound debt management strategy and rigorous evaluation of the quality and feasibility of infrastructure projects to ensure healthy rates of return on investment.

\section{BACKGROUND}

1. Tanzania's macroeconomic outcomes improved substantially over the last decade with sustained high rates of growth and relatively low inflation. Prior to the global financial crisis, growth had been accelerating (averaging 7 percent per year during 2002-2009), which, together with a sharp increase in revenue collection and increased donor funding, provided room for a substantial expansion in public spending. Inflation was kept in check for much of this period, but accelerated in 2008 (13.5 percent at end year), driven mainly by lagged effects of the spike in international food and fuel prices, and more recently, by adverse regional food supply shocks. Growth slowed as the global crisis hit, but the government's economic recovery program cushioned the impact on the economy. Economic indicators suggest that growth began to accelerate in the second half of 2009, stemming from good performance in agriculture, construction, and, transport and communication. Tanzania also achieved a large buildup in official international reserves, partly reflecting solid export growth, Fund balance of payments support under the Exogenous Shocks

\footnotetext{
${ }^{1}$ Prepared by the IMF and World Bank staff in consultation with the authorities. This DSA replaces the one prepared in May (Country Report No. 09/179). Tables and Figures are in fiscal years (July-June). For example, 2009 refers to fiscal year 2009/10.
} 
Facility (ESF), and the SDR allocations. At end-2009, gross international reserves were about US $\$ 3.5$ billion, more than 5 months of current year imports of goods and services.

2. Tanzania has benefited from extensive debt relief under the HIPC Initiative and, more recently, the Multilateral Debt Relief Initiative (MDRI). HIPC and MDRI debt relief reduced Tanzania's debt burden sharply, with all debt indicators declining to levels well below risk thresholds. Debt policy has remained prudent since the provision of debt relief, with total external debt declining from US\$7.1 billion, or 57.3 percent of GDP at end-June 2006 before MDRI went into effect, to US\$3.7 billion, or 24.9 percent of GDP at end-June 2008. Total external debt increased to US $\$ 4.5$ billion, or 25.6 percent of GDP by end-June $2009 .^{2}$ In present value (PV) terms, the public and publicly guaranteed sector (PPG) external debt stood at about 12 percent of GDP at end-June 2009, or 541/2 percent of expots (end-June 2008 figures were 10.6, and 44.9 percent respectively). ${ }^{3}$ Public external debt service was around 1.0 percent of exports of goods and services in 2008/9, compared to 1.7 percent in 2007/8. New external borrowing, concentrated on financing social investment primarily in the areas of education and health, was contracted on highly concessional terms. At end-June 2009, the composition of PPG external debt was 61 percent to multilateral and 20 percent to bilateral creditors. Tanzania's public domestic debt increased slightly from TSh 3.3 trillion (14.3 percent of GDP) at end-June 2008 to TSh 3.5 trillion (13.0 percent of GDP) at end-June 2009, about half of which was short-term Treasury bills.

3. The authorities' medium-term policy is focused on stepping up public investment. The draft poverty reduction strategy (MKUKUTA II), which will cover 2010-2015, is expected to reinforce emphasis on growth through stepping up the infrastructure investment. Increased infrastructure spending, especially in transportation, power generation, and irrigation, can play a critical role in stimulating sectors vital to growth both in Tanzania and the region. Financing for the additional public spending is expected to come from a combination of domestic and external borrowing. While concessional borrowing and grants will remain the main source of financing for investment spending, these resources are unlikely to be sufficient for a significant scaling up of infrastructure investment. The authorities plan to explore sources for semi-concessional and nonconcessional external borrowing, domestic borrowing, and to seek greater participation of the private sector in financing and/or implementing infrastructure projects through Public Private Partnership (PPP) arrangements.

\section{BASEline Assumptions}

\section{Baseline assumptions differ somewhat from those in the previous DSA to reflect} additional public investment spending. The baseline scenario envisages a gradual recovery in private sector activity over the next three years from the current slowdown caused by the global

\footnotetext{
${ }^{2}$ The debt stock excludes the estimated amount of interest arrears of about US\$560 million, which are expected to be canceled upon conclusion of formal agreements on HIPC debt relief.

${ }^{3}$ PPG debt at this stage only reflects central government debt. The authorities are cataloguing contingent liabilities, including government guarantees to state-owned enterprises.
} 
crisis. Growth in $2009 / 10$ is projected to be 5.8 percent, increasing to 6.5 percent in $2010 / 11$, and 7.1 percent in 2011/12 before reaching 7.5 percent in 2012/13 and thereafter. Compared with the 2009 Joint IMF-World Bank DSA, the current baseline scenario assumes a higher growth path over the long term, 7.5 compared to 6 percent after 2020/21, reflecting the stepping up of infrastructure investment financed via additional domestic and external borrowing on less concessional terms (Box 1). Inadequate infrastructure is considered a key constraint to capacity building, the business environment, productivity and subsequently higher growth in the country and in the region. ${ }^{4}$ The authorities have developed a list of priority high return infrastructure projects, which are expected to produce synergistic growth effects on vital sectors. Inflation (GDP deflator) is projected at the Bank of Tanzania's medium-term objective of 5 percent, the real exchange rate is assumed to stay constant at 2009/10 levels throughout the projection period, and export/import price index deflators are assumed to grow 2 percent annually (these assumptions remain unchanged from the previous DSA). The current account deficit is expected to increase slightly in 2010/11, before improving steadily to 3.6 percent of GDP in the long term, due to the supply response to more infrastructure.

Box 1. Comparison with 2009 DSA
-
-
-
Exowth: The current DSA assumes a higher growth path compared to the 2009 DSA (7.5
Exports and imports: Growth of exports and imports over the long term is somewhat higher
than the 2009 DSA to reflect higher infrastructure spending and growth.
Investment spending: The elevated infrastructure investment is reflected in an initial increase
in investment spending from 8.8 percent of GDP in $2008 / 09$ to an average of 9.7 percent during
2010-2013, and thereafter 9.4 percent. The previous DSA reflected a slightly larger percentage in
the initial year - due to lower GDP - and smaller percentages in the outer years.
Public deficit: The public sector deficit increases in the near term on account of additional
public investment before steadily dropping to 2.8 percent of GDP by $2029 / 30$. In the 2009 DSA
the declining path was steeper with the deficit reaching 1.4 percent by end of the projection
period.
Foreign concessional loans and grants: In the current baseline scenario external grants decline
to about 3 percent of GDP by $2019 / 20$ and thereafter (compared to 4.5 percent in the 2009 DSA)
while foreign concessional loans decline at the same rate as in the previous DSA, to reflect
gradual reduction in aid dependency.

5. To finance additional infrastructure spending, the baseline incorporates domestic and external borrowing on non-concessional terms during the upcoming PSI program period. The additional borrowing includes non-concessional external financing of US\$1.5 billion over three years (US\$525 million disbursed in 2010/11, 2011/12, and US\$450 million in 2012/13) and 1 percent of GDP for the remainder of the projection period. It also includes domestic financing of about 1 percent of GDP for 2010/11-2014/15. The new domestic borrowing is assumed to be at an

\footnotetext{
${ }^{4}$ See IMF Working Paper 08/256 “Creating Sustainable Fiscal Space for Infrastructure: the Case of Tanzania," IMF Board paper "Public Investment and Fiscal Policy", and Africa's infrastructure: a time for transformation (World Bank, 2010).
} 
interest rate of 10 percent (with automatic rollover), while external borrowing is assumed to have an average interest rate of 9 percent, with a 1-year grace period and 10-years' maturity. The terms for external borrowing were set high, at market rates, to mirror a possible premium for a Eurobond. The authorities are planning a Eurobond, likely in FY 2011/12, and will explore other options for semiconcessional financing, e.g. IBRD.

\section{Government revenues as a percent of GDP are assumed to increase — albeit more} gradually than in recent years - while external grants and concessional loans will decline. Domestic revenues are assumed to increase from 151/2 percent of GDP in 2009/10 to about 20 percent of GDP by $2017 / 18$ and gradually reach 21.5 percent by $2029 / 30$ — within IMF staff estimates of tax potential. On the other hand, external grants are assumed to decline from 6 percent of GDP in 2009/10 to about 3 percent of GDP by 2019/20 and remain roughly at that level. ${ }^{5,6}$ External concessional loans (including both program and project loans) are assumed to fall from 4.2 percent of GDP in 2009/10 to about 2.0 percent of GDP by 2029/30, consistent with a gradual reduction in Tanzania's aid dependency. Annual development spending is assumed to increase to 9.7 percent of GDP between 2010 and 2013, reflecting the enhanced public investment, and then stabilize at 9.4 percent of GDP for the rest of the projection period. The baseline also accounts for annual maintenance costs - added to recurrent expenditure - of 5 percent of the total value of the accumulated additional infrastructure spending. The maintenance costs reach about 0.2 percent of GDP in 2014/15, before gradually declining relative to GDP over the remainder of the projection period. Any residual financing need beyond 2014/15 is assumed to be met by domestic borrowing, which under the baseline would not exceed 1.8 percent of GDP.

\section{Institutional mechanisms to ensure the quality and feasibility of new infrastructure} spending and an appropriate risk management strategy are called for. The authorities have developed a list of priority projects consistent with strategic planning documents, including the Medium Term Public Sector Investment Plan and the new PRS. The IMF and World Bank have recently provided technical assistance on debt management and institutional and legal frameworks for PPPs. Drawing on this assistance, the authorities are preparing a new medium-term debt strategy and a new PPP framework, both of which should be in place by end-2010. Similarly, the President has established a task force, which has developed recommendations to improve the business environment, and thus, the private sector response to scaled up infrastructure investment.

\section{EXternal Debt Sustainability Analysis}

\section{The risk of external debt distress is low under the baseline scenario despite the} proposed increase in infrastructure spending. An increase in non-concessional external borrowing of US\$ 1.5 billion over the 3-year period 2010-13, reflecting the authorities' specific

\footnotetext{
${ }^{5}$ External grants in 2009/10 were significantly higher than the recent historical average of 4-5 percent, reflecting extraordinary support to mitigate the effects of the global crisis.

${ }^{6}$ The assumed path for grants does not build in any additional resources related to climate change initiatives.
} 
investment plan to improve transportation infrastructure, and 1 percent of GDP for the remaining projection period, as an assumption for potential future investment, will increase debt indicators but should not jeopardize long-run sustainability (Figure 1). Debt service indicators increase gradually throughout the projection period, but stay well below risk thresholds. The PV of public external debt-to-GDP ratio would increase from 13.1 percent of GDP in 2009/10 to 22.7 percent by 2015, before falling to 21.5 percent by end of projection period (in the previous DSA, these ratios were $11.7,16.2$, and 7.1, respectively). The PV of debt-to-exports is expected to peak at 90.1 percent of GDP in 2013/14, before declining to 71.1 percent in 2029/30. The debt service-to-exports ratio would reach 9.6 percent by $2029 / 30$ - debt service indicators would continue to increase based on a conservative assumption that new financing would be on the most expensive terms. (In fact, the authorities do plan to maximize concessionality of the new borrowing, even if it does not meet the 35 percent grant element threshold.) The sensitivity analysis indicates that Tanzania's public external debt would remain below risk thresholds applicable to Tanzania under all standard bound tests and extreme shocks. ${ }^{7}$ The alternative scenario with less favorable financing terms shows the strongest deterioration in most indicators, but they do not breach the risk thresholds. Indeed, thebaseline already reflect this scenario to some extent, given its highly conservative assumptions on terms for non-concessional financing.

9. The alternative high investment-low growth scenario indicates, however, that Tanzania's external debt dynamics is sensitive to the assumption on real GDP growth. An alternative downside scenario that assumes a permanently lower growth rate of 6 percent is considered (A3 in Table 2 and Figure 1). This scenario could also be interpreted as reflecting no measurable impact on growth of the additional infrastructure investment. This alternative scenario results in a PV of debt-to-GDP ratio of 27 percent by 2030, which is higher than the baseline scenario. In addition, the historical scenario (A1 in Table 3), under which key variables are held constant at historical averages, yields indicators that increase substantially towards the end of the projection period. The primary reason for this result is that the baseline builds in higher rates of growth, consistent with higher levels of public investment. These alternative scenarios again highlight the need for institutions to support a sound debt management strategy and strong returns to infrastructure investment.

\section{Fiscal Debt Sustainability Analysis}

\section{The path of total public debt, which includes external debt and domestic debt, also} appears to be sustainable, but is sensitive to fiscal adjustment. The PV of public debt-to-GDP ratio increases from 26.9 in $2009 / 10$ to 34.1 by 2029/30, while debt service-to-revenue increases quite sharply from 7.9 to 19.8 by end of the projection period (Figure 2).

\footnotetext{
7 The World Bank's Country Policy and Institutional Assessment (CPIA) ranks Tanzania as a "strong performer," with a 2006-08 CPIA of 3.89. Debt burden thresholds for strong performers are PV of debt to GDP ratio of 50 percent, PV of debt-to-exports ratio of 200 percent, $\mathrm{PV}$ of debt-to-revenue ratio of 300 percent, debt-service-to-exports ratio of 25 percent, and debt-service-to-revenue ratio of 35 percent.
} 
11. Under the alternative scenario with permanently low growth (A3 in Table 4), the PV of public debt would reach 40 percent of GDP by 2029/30, while the debt service-to-revenue ratio would experience a relatively large and sustained increase reaching 27 percent. Also, compared to the previous DSA, the sensitivity analysis holding the primary deficit at the current level shows relative deterioration in debt indicators, reflecting the FY09/10 fiscal expansion in response to the global crisis. If the primary fiscal balance remained unchanged from 2010 onwards (A2 in Table 4), debt service payments would absorb 51 percent of revenue by 2029/30. It is expected, however, that the expanded fiscal spending will be phased out as the time-bound discretionary components are withdrawn and automatic stabilizers reverse. The most extreme stress test is an exchange rate depreciation shock (one-time 30 percent real depreciation in 2011), resulting in an increase of the PV of public debt-to-GDP ratio from 26.9 in 2009/10 to 43.9 by 2029/30, still below the risk threshold.

\section{Conclusion}

\section{Based on the updated baseline scenario, Tanzania's debt indicators are expected to} remain well below indicative, country-specific thresholds. The additional borrowing to finance a stepping up of infrastructure investment over the medium term will increase the present value (PV) of debt-to-GDP and other indicators, but should not jeopardize long-term debt sustainability, particularly assuming a modest growth response to the infrastructure investment. Debt indicators are sensitive to a number of parameters, but in general the downside scenarios and standard sensitivity analysis support the assessment of a low risk of debt distress. Nevertheless, a sound debt management strategy, a conservative approach to non-concessional borrowing, and rigorous evaluation of the quality and feasibility of investment projects are important factors for maintaining debt and fiscal sustainability. For example, should key variables such as donor support or real GDP growth deteriorate excessively, government spending would have to adjust to maintain fiscal and debt sustainability. 
Table 1.: External Debt Sustainability Framework, Baseline Scenario, 2007-2030 1/

In percent of GDP, unless otherw

\begin{tabular}{|c|c|c|c|c|c|c|c|c|c|c|c|c|c|c|c|}
\hline & \multicolumn{3}{|c|}{ Actual } & \multirow{2}{*}{\multicolumn{2}{|c|}{$\begin{array}{lll}\text { Historical } & 0 & \text { Standard } \\
\text { Average } & 0 & \text { Deviation }\end{array}$}} & \multicolumn{6}{|c|}{ Projections } & \multirow{2}{*}{$\begin{array}{c}2010-2015 \\
\text { Average } \\
\end{array}$} & \multirow[b]{2}{*}{2020} & \multirow[b]{2}{*}{2030} & \multirow{2}{*}{$\begin{array}{r}\begin{array}{r}2016-2030 \\
\text { Average }\end{array} \\
\end{array}$} \\
\hline & 2007 & 2008 & 2009 & & & 2010 & 2011 & 2012 & 2013 & 2014 & 2015 & & & & \\
\hline External debt (nominal) 1/ & 20.8 & 24.9 & 25.6 & & & 26.6 & 29.5 & 32.0 & 33.6 & 34.4 & 35.2 & & 34.4 & 34.7 & \\
\hline $\mathrm{o} / \mathrm{w}$ public and publicly guaranteed (PPG) & 17.0 & 21.0 & 21.1 & & & 22.4 & 25.3 & 27.6 & 29.1 & 29.7 & 30.3 & & 28.1 & 24.3 & \\
\hline Change in external debt & -33.6 & 4.1 & 0.7 & & & 1.0 & 2.9 & 2.5 & 1.6 & 0.8 & 0.9 & & -0.2 & 0.3 & \\
\hline Identified net debt-creating flows & 2.5 & 2.6 & 4.2 & & & 4.5 & 4.2 & 3.8 & 3.4 & 2.3 & 2.0 & & 0.4 & -2.9 & \\
\hline Non-interest current account deficit & 9.7 & 10.3 & 9.6 & 6.6 & 2.7 & 8.1 & 8.4 & 8.3 & 8.1 & 7.5 & 7.4 & & 6.3 & 3.6 & 5.5 \\
\hline Deficit in balance of goods and services & 13.0 & 14.0 & 12.9 & & & 11.3 & 10.5 & 10.3 & 10.1 & 9.5 & 9.5 & & 8.1 & 5.0 & \\
\hline Exports & 24.5 & 23.6 & 22.5 & & & 22.4 & 24.1 & 24.2 & 24.2 & 24.5 & 25.1 & & 26.7 & 30.3 & \\
\hline Imports & 37.4 & 37.6 & 35.4 & & & 33.7 & 34.6 & 34.5 & 34.3 & 34.0 & 34.6 & & 34.8 & 35.3 & \\
\hline Net current transfers (negative $=$ inflow) & -3.5 & -3.6 & -3.4 & -3.0 & 1.3 & -3.3 & -2.5 & -2.4 & -2.2 & -2.1 & -2.0 & & -1.5 & -0.9 & -1.4 \\
\hline $\mathrm{o} / \mathrm{w}$ official & -3.3 & -3.5 & -3.3 & & & -3.2 & -2.4 & -2.2 & -2.1 & -1.9 & -1.9 & & -1.4 & -0.9 & \\
\hline Other current account flows (negative = net inflow) & 0.2 & -0.1 & 0.1 & & & 0.1 & 0.5 & 0.3 & 0.2 & 0.1 & -0.1 & & -0.2 & -0.5 & \\
\hline Net FDI (negative = inflow) & -4.3 & -3.7 & -2.8 & -3.7 & 0.4 & -2.4 & -2.8 & -2.9 & -3.0 & -3.4 & -3.7 & & -4.4 & -5.2 & -4.6 \\
\hline Endogenous debt dynamics $2 /$ & -2.9 & -4.0 & -2.6 & & & -1.2 & -1.4 & -1.6 & -1.7 & -1.7 & -1.8 & & -1.5 & -1.3 & \\
\hline Contribution from nominal interest rate & 0.2 & 0.2 & 0.2 & & & 0.2 & 0.1 & 0.4 & 0.5 & 0.6 & 0.6 & & 0.8 & 1.1 & \\
\hline Contribution from real GDP growth & -3.6 & -1.2 & -1.4 & & & -1.4 & -1.6 & -1.9 & -2.2 & -2.3 & -2.4 & & -2.4 & -2.4 & \\
\hline Contribution from price and exchange rate changes & 0.5 & -3.0 & -1.4 & & & & & & & & & & & & \\
\hline Residual (3-4) $3 /$ & -36.1 & 1.4 & -3.5 & & & -3.6 & -1.3 & -1.3 & -1.8 & -1.5 & -1.1 & & -0.6 & 3.2 & \\
\hline $\mathrm{o} / \mathrm{w}$ exceptional financing & 0.0 & 0.0 & 0.0 & & & 0.0 & 0.0 & 0.0 & 0.0 & 0.0 & 0.0 & & 0.0 & 0.0 & \\
\hline PV of external debt $4 /$ & $\ldots$ & $\ldots$ & 16.8 & & & 17.3 & 21.0 & 24.2 & 25.9 & 26.7 & 27.7 & & 29.0 & 32.0 & \\
\hline In percent of exports & $\ldots$ & $\ldots$ & 74.5 & & & 76.9 & 86.9 & 100.1 & 106.9 & 109.2 & 110.5 & & 108.4 & 105.4 & \\
\hline PV of PPG external debt & $\ldots$ & ... & 12.2 & & & 13.1 & 16.7 & 19.8 & 21.4 & 22.1 & 22.7 & & 22.6 & 21.5 & \\
\hline In percent of exports & $\ldots$ & $\ldots$ & 54.4 & & & 58.4 & 69.4 & 82.1 & 88.2 & 90.1 & 90.6 & & 84.6 & 71.1 & \\
\hline In percent of government revenues & ... & ... & 80.9 & & & 84.5 & 104.6 & 121.6 & 128.7 & 130.7 & 127.5 & & 111.0 & 100.1 & \\
\hline Debt service-to-exports ratio (in percent) & 2.0 & 4.2 & 4.5 & & & 3.2 & 3.3 & 4.3 & 5.5 & 6.4 & 7.3 & & 9.9 & 12.9 & \\
\hline PPG debt service-to-exports ratio (in percent) & 1.1 & 1.7 & 1.0 & & & 1.0 & 1.2 & 2.3 & 3.6 & 4.6 & 5.4 & & 7.6 & 9.6 & \\
\hline PPG debt service-to-revenue ratio (in percent) & 1.9 & 2.6 & 1.4 & & & 1.5 & 1.9 & 3.4 & 5.3 & 6.7 & 7.6 & & 10.0 & 13.5 & \\
\hline Total gross financing need (Billions of U.S. dollars) & 0.9 & 1.4 & 1.7 & & & 1.5 & 1.7 & 1.8 & 2.0 & 1.9 & 2.0 & & 2.6 & 3.2 & \\
\hline Non-interest current account deficit that stabilizes debt ratio & 43.3 & 6.2 & 8.8 & & & 7.1 & 5.5 & 5.8 & 6.5 & 6.7 & 6.6 & & 6.6 & 3.3 & \\
\hline \multicolumn{16}{|l|}{ Key macroeconomic assumptions } \\
\hline Real GDP growth (in percent) & 6.9 & 7.3 & 6.4 & 7.0 & 0.4 & 5.8 & 6.5 & 7.1 & 7.5 & 7.5 & 7.5 & 7.0 & 7.5 & 7.5 & 7.5 \\
\hline GDP deflator in US dollar terms (change in percent) & -0.9 & 16.7 & 6.0 & 2.5 & 6.8 & 3.6 & 1.9 & 1.7 & 1.9 & 1.9 & 0.4 & 1.9 & 1.9 & 1.9 & 1.9 \\
\hline Effective interest rate (percent) $5 /$ & 0.3 & 1.1 & 1.1 & 0.8 & 0.4 & 0.8 & 0.6 & 1.4 & 1.8 & 1.9 & 2.0 & 1.4 & 2.6 & 3.4 & 2.8 \\
\hline Growth of exports of G\&S (US dollar terms, in percent) & 19.1 & 21.0 & 7.5 & 15.6 & 8.7 & 9.2 & 16.7 & 9.0 & 9.8 & 10.8 & 10.7 & 11.0 & 10.9 & 10.9 & 10.9 \\
\hline Growth of imports of G\&S (US dollar terms, in percent) & 21.5 & 25.8 & 6.3 & 13.5 & 12.3 & 4.4 & 11.3 & 8.4 & 9.0 & 8.6 & 9.9 & 8.6 & 9.6 & 9.6 & 9.6 \\
\hline Grant element of new public sector borrowing (in percent) & & & & & $\ldots$ & 49.2 & 18.7 & 16.5 & 18.5 & 21.8 & 17.6 & 23.7 & 4.4 & -2.9 & 2.1 \\
\hline Government revenues (excluding grants, in percent of GDP) & 14.4 & 15.9 & 15.1 & & & 15.5 & 16.0 & 16.3 & 16.6 & 16.9 & 17.8 & & 20.4 & 21.5 & 20.6 \\
\hline Aid flows (in Billions of US dollars) 7/ & 0.8 & 1.3 & 1.0 & & & 2.1 & 1.9 & 1.9 & 2.0 & 2.1 & 2.2 & & 2.5 & 6.1 & \\
\hline $\mathrm{o} / \mathrm{w}$ Grants & 0.8 & 1.3 & 1.0 & & & 1.4 & 1.2 & 1.3 & 1.3 & 1.4 & 1.5 & & 1.8 & 4.5 & \\
\hline $\mathrm{o} / \mathrm{w}$ Concessional loans & 0.0 & 0.0 & 0.0 & & & 0.6 & 0.7 & 0.7 & 0.7 & 0.7 & 0.7 & & 0.7 & 1.5 & \\
\hline Grant-equivalent financing (in percent of GDP) $8 /$ & $\ldots$ & $\ldots$ & $\ldots$ & & & 7.7 & 5.8 & 5.3 & 5.0 & 5.0 & 4.8 & & 3.3 & 3.1 & 3.4 \\
\hline Grant-equivalent financing (in percent of external financing) $8 /$ & $\ldots$ & $\ldots$ & $\ldots$ & & & 83.0 & 59.7 & 58.0 & 59.3 & 63.1 & 62.6 & & 52.6 & 44.3 & 50.9 \\
\hline \multicolumn{16}{|l|}{ Memorandum items: } \\
\hline DP (Billions of US dollars) & 15.2 & 19.0 & 21.5 & & & 23.5 & 25.5 & 27.8 & 30.5 & 33.4 & 36.0 & & 56.7 & 140.3 & \\
\hline Nominal dollar GDP growth & 6.0 & 25.2 & 12.8 & & & 9.6 & 8.5 & 8.9 & 9.6 & 9.6 & 8.0 & 9.0 & 9.5 & 9.5 & 9.5 \\
\hline PV of PPG external debt (in Billions of US dollars) & & & 2.6 & & & 3.0 & 4.2 & 5.4 & 6.4 & 7.3 & 8.1 & & 12.6 & 29.5 & \\
\hline (PVt-PVt-1)/GDPt-1 (in percent) & & & & & & 2.0 & 5.0 & 4.8 & 3.5 & 2.8 & 2.5 & 3.4 & 1.9 & 2.0 & 2.0 \\
\hline Gross remittances (Billions of US dollars) & & & & & & & & & & & & & & & \\
\hline PV of PPG external debt (in percent of GDP + remittances) & $\ldots$ & $\ldots$ & 12.2 & & & 13.1 & 16.7 & 19.8 & 21.4 & 22.1 & 22.7 & & 22.6 & 21.5 & \\
\hline PV of PPG external debt (in percent of exports + remittances) & $\ldots$ & $\ldots$ & 54.4 & & & 58.4 & 69.4 & 82.1 & 88.2 & 90.1 & 90.6 & & 84.6 & 71.1 & \\
\hline Debt service of PPG external debt (in percent of exports + remittances) & $\ldots$ & $\ldots$ & 1.0 & & & 1.0 & 1.2 & 2.3 & 3.6 & 4.6 & 5.4 & & 7.6 & 9.6 & \\
\hline
\end{tabular}

1/ Includes both public and private sector external debt.

id debt ratio, with $\mathrm{r}=$ nominal interest rate; $\mathrm{g}=$ real GDP growth rate, and $\rho=$ growth rate of GDP deflator in U.S. dollar terms.

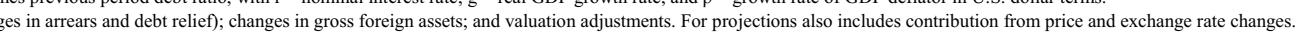

4/ Assumes that PV of private sector debt is equivalent to its face value.
$5 /$ Current-year interest payments divided by previous period debt stock

$6 /$ Historical averages and standard deviations are generally derived over the past 10 years, subject to data availability.

$7 /$ Defined as grants, concessional loans, and debt relief.

8/ Grant-equivalent financing includes grants provided directly to the government and through new borrowing (difference between the face value and the PV of new debt). 
Table 2.Tanzania: Sensitivity Analysis for Key Indicators of Public and Publicly Guaranteed External Debt, 2010-2030 (In percent)

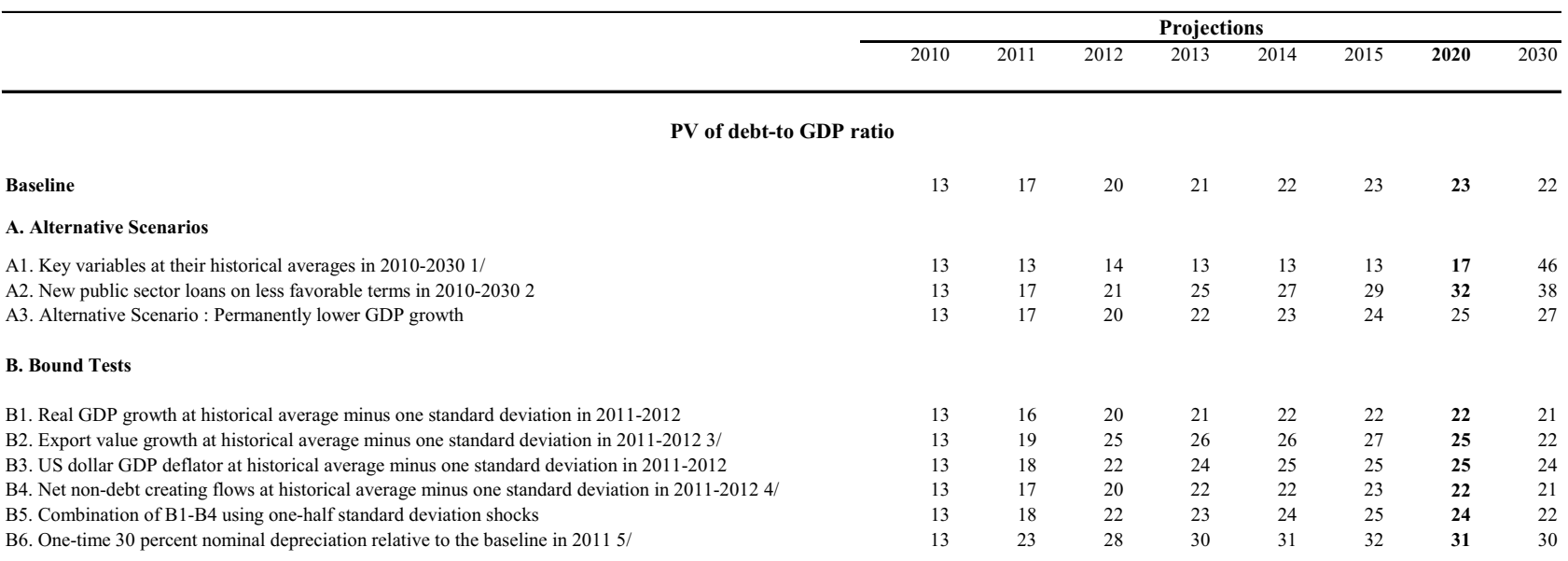

PV of debt-to-exports ratio

Baseline

A. Alternative Scenarios

A1. Key variables at their historical averages in 2010-2030 1/

A2. New public sector loans on less favorable terms in 2010-2030 2

A3. Alternative Scenario : Permanently lower GDP growth

B. Bound Tests

B1. Real GDP growth at historical average minus one standard deviation in 2011-2012

B2. Export value growth at historical average minus one standard deviation in 2011-2012 3/

B3. US dollar GDP deflator at historical average minus one standard deviation in 2011-2012

B4. Net non-debt creating flows at historical average minus one standard deviation in 2011-2012 4/

B5. Combination of B1-B4 using one-half standard deviation shocks

B6. One-time 30 percent nominal depreciation relative to the baseline in 2011 5/

PV of debt-to-revenue ratio

Baseline

A. Alternative Scenarios

A1. Key variables at their historical averages in 2010-2030 1/

A2. New public sector loans on less favorable terms in 2010-2030 2

A3. Alternative Scenario : Permanently lower GDP growth

\section{B. Bound Tests}

B1. Real GDP growth at historical average minus one standard deviation in 2011-2012

B2. Export value growth at historical average minus one standard deviation in 2011-2012 3/

B3. US dollar GDP deflator at historical average minus one standard deviation in 2011-2012

B4. Net non-debt creating flows at historical average minus one standard deviation in 2011-2012 4/

B5. Combination of B1-B4 using one-half standard deviation shocks

B6. One-time 30 percent nominal depreciation relative to the baseline in $20115 /$

$\begin{array}{rrrrrrrr}85 & 103 & 120 & 127 & 129 & 126 & \mathbf{1 0 9} & 97 \\ 85 & 118 & 151 & 156 & 156 & 151 & \mathbf{1 2 3} & 101 \\ 85 & 110 & 136 & 144 & 146 & 142 & \mathbf{1 2 3} & 110 \\ 85 & 105 & 123 & 130 & 132 & 128 & \mathbf{1 1 0} & 97 \\ 85 & 111 & 134 & 141 & 142 & 138 & \mathbf{1 1 8} & 103 \\ 85 & 146 & 170 & 180 & 183 & 178 & \mathbf{1 5 4} & 137\end{array}$


Table 2.Tanzania: Sensitivity Analysis for Key Indicators of Public and Publicly Guaranteed External Debt, 2010-2030 (continued)

(In percent)

Debt service-to-exports ratio

Baseline

A. Alternative Scenarios

A1. Key variables at their historical averages in 2010-2030 1/

A2. New public sector loans on less favorable terms in 2010-2030 2

A3. Alternative Scenario : Permanently lower GDP growth

\section{B. Bound Tests}

B1. Real GDP growth at historical average minus one standard deviation in 2011-2012

B2. Export value growth at historical average minus one standard deviation in 2011-2012 3/

B3. US dollar GDP deflator at historical average minus one standard deviation in 2011-2012

B4. Net non-debt creating flows at historical average minus one standard deviation in 2011-2012 4/

B5. Combination of B1-B4 using one-half standard deviation shocks

B6. One-time 30 percent nominal depreciation relative to the baseline in 20115 /

\section{Debt service-to-revenue ratio}

Baseline

A. Alternative Scenarios

A1. Key variables at their historical averages in 2010-2030 1/

A2. New public sector loans on less favorable terms in 2010-2030 2

A3. Alternative Scenario : Permanently lower GDP growth

B. Bound Tests

B1. Real GDP growth at historical average minus one standard deviation in 2011-2012 B2. Export value growth at historical average minus one standard deviation in 2011-2012 3/

B3. US dollar GDP deflator at historical average minus one standard deviation in 2011-2012

B4. Net non-debt creating flows at historical average minus one standard deviation in 2011-2012 4/

B5. Combination of B1-B4 using one-half standard deviation shocks

B6. One-time 30 percent nominal depreciation relative to the baseline in 20115

Memorandum item

Grant element assumed on residual financing (i.e., financing required above baseline) 6/

1

1

2

4

(20)

$\begin{array}{ll}3 & 4 \\ 3 & 4 \\ 5 & \end{array}$

Sources: Country authorities; and staff estimates and projections.

1/ Variables include real GDP growth, growth of GDP deflator (in U.S. dollar terms), non-interest current account in percent of GDP, and non-debt creating flows.

$2 /$ Assumes that the interest rate on new borrowing is by 2 percentage points higher than in the baseline., while grace and maturity periods are the same as in the baseline.

3/ Exports values are assumed to remain permanently at the lower level, but the current account as a share of GDP is assumed to return to its baseline level after the shock (implicitly assuming an offsetting adjustment in import levels).

4/ Includes official and private transfers and FDI.

5/ Depreciation is defined as percentage decline in dollar/local currency rate, such that it never exceeds 100 percent.

6/ Applies to all stress scenarios except for A2 (less favorable financing) in which the terms on all new financing are as specified in footnote 2. 
Table 3.Tanzania: Public Sector Debt Sustainability Framework, Baseline Scenario, 2007-2030

(In percent of GDP, unless otherwise indicated)

\begin{tabular}{|c|c|c|c|c|c|c|c|c|c|c|c|c|c|c|c|}
\hline & \multicolumn{3}{|c|}{ Actual } & \multirow[b]{2}{*}{ Average } & \multirow[b]{2}{*}{$\begin{array}{l}\text { Standard } \\
\text { Deviation } \\
\end{array}$} & \multicolumn{5}{|l|}{ Estimate } & \multicolumn{5}{|c|}{ Projections } \\
\hline & 2007 & 2008 & 2009 & & & 2010 & 2011 & 2012 & 2013 & 2014 & 2015 & $\begin{array}{l}2010-15 \\
\text { Average }\end{array}$ & 2020 & 2030 & $\begin{array}{c}2016-30 \\
\text { Average }\end{array}$ \\
\hline $\begin{array}{l}\text { Public sector debt 1/ } \\
\text { o/w foreign-currency denominated }\end{array}$ & $\begin{array}{l}39.4 \\
17.0\end{array}$ & $\begin{array}{l}35.3 \\
21.0\end{array}$ & $\begin{array}{l}34.4 \\
21.1\end{array}$ & & & $\begin{array}{l}36.2 \\
22.4\end{array}$ & $\begin{array}{l}37.9 \\
25.3\end{array}$ & $\begin{array}{l}39.2 \\
27.6\end{array}$ & $\begin{array}{l}40.1 \\
29.1\end{array}$ & $\begin{array}{l}40.5 \\
29.7\end{array}$ & $\begin{array}{l}41.0 \\
30.3\end{array}$ & & $\begin{array}{l}36.8 \\
28.1\end{array}$ & $\begin{array}{l}36.9 \\
24.3\end{array}$ & \\
\hline Change in public sector debt & -28.3 & -4.2 & -0.9 & & & 1.8 & 1.7 & 1.3 & 0.9 & 0.5 & 0.5 & & -0.6 & 0.2 & \\
\hline Identified debt-creating flows & -4.8 & -4.6 & -1.1 & & & 2.9 & 2.9 & 2.3 & 1.7 & 1.1 & 1.0 & & -0.7 & -0.7 & \\
\hline Primary deficit & 2.8 & -1.2 & 3.6 & 1.8 & 1.5 & 4.5 & 4.9 & 4.4 & 4.0 & 3.4 & 2.6 & 4.0 & 0.8 & -0.2 & 0.5 \\
\hline Revenue and grants & 19.4 & 22.9 & 19.8 & & & 21.7 & 20.9 & 20.9 & 20.8 & 21.1 & 22.0 & & 23.6 & 24.8 & \\
\hline of which: grants & 5.0 & 6.9 & 4.7 & & & 6.1 & 4.9 & 4.5 & 4.2 & 4.2 & 4.2 & & 3.2 & 3.2 & \\
\hline Primary (noninterest) expenditure & 22.2 & 21.7 & 23.4 & & & 26.1 & 25.8 & 25.3 & 24.9 & 24.5 & 24.6 & & 24.4 & 24.6 & \\
\hline Automatic debt dynamics & -7.7 & -3.5 & -4.6 & & & -1.5 & -1.9 & -2.1 & -2.4 & -2.3 & -1.7 & & -1.5 & -0.5 & \\
\hline Contribution from interest rate/growth differential & -4.3 & -4.0 & -2.7 & & & -1.3 & -1.9 & -2.2 & -2.4 & -2.3 & -2.1 & & -1.5 & -0.5 & \\
\hline of which: contribution from average real interest rate & 0.1 & -1.4 & -0.5 & & & 0.6 & 0.3 & 0.4 & 0.4 & 0.5 & 0.8 & & 1.1 & 2.1 & \\
\hline of which: contribution from real GDP growth & -4.4 & -2.7 & -2.1 & & & -1.9 & -2.2 & -2.5 & -2.7 & -2.8 & -2.8 & & -2.6 & -2.6 & \\
\hline Contribution from real exchange rate depreciation & -3.4 & 0.6 & -1.9 & & & -0.2 & 0.0 & 0.1 & 0.0 & 0.0 & 0.4 & & $\ldots$ & $\ldots$ & \\
\hline Other identified debt-creating flows & 0.0 & 0.0 & -0.2 & & & 0.0 & 0.0 & 0.0 & 0.0 & 0.0 & 0.0 & & 0.0 & 0.0 & \\
\hline Privatization receipts (negative) & 0.0 & 0.0 & -0.2 & & & 0.0 & 0.0 & 0.0 & 0.0 & 0.0 & 0.0 & & 0.0 & 0.0 & \\
\hline Recognition of implicit or contingent liabilities & 0.0 & 0.0 & 0.0 & & & 0.0 & 0.0 & 0.0 & 0.0 & 0.0 & 0.0 & & 0.0 & 0.0 & \\
\hline Debt relief (HIPC and other) & 0.0 & 0.0 & 0.0 & & & 0.0 & 0.0 & 0.0 & 0.0 & 0.0 & 0.0 & & 0.0 & 0.0 & \\
\hline Other (specify, e.g. bank recapitalization) & 0.0 & 0.0 & 0.0 & & & 0.0 & 0.0 & 0.0 & 0.0 & 0.0 & 0.0 & & 0.0 & 0.0 & \\
\hline Residual, including asset changes & -23.5 & 0.5 & 0.3 & & & -1.1 & -1.2 & -1.0 & -0.8 & -0.6 & -0.5 & & 0.1 & 0.9 & \\
\hline \multicolumn{16}{|l|}{ Other Sustainability Indicators } \\
\hline PV of public sector debt & 22.5 & 14.3 & 25.6 & & & 26.9 & 29.4 & 31.4 & 32.4 & 32.9 & 33.5 & & 31.4 & 34.1 & \\
\hline $\mathrm{o} / \mathrm{w}$ foreign-currency denominated & 0.0 & 0.0 & 12.2 & & & 13.1 & 16.7 & 19.8 & 21.4 & 22.1 & 22.7 & & 22.6 & 21.5 & \\
\hline $\mathrm{o} / \mathrm{w}$ external & $\ldots$ & $\ldots$ & 12.2 & & & 13.1 & 16.7 & 19.8 & 21.4 & 22.1 & 22.7 & & 22.6 & 21.5 & \\
\hline $\mathrm{PV}$ of contingent liabilities (not included in public sector debt) & & $\ldots$ & $\ldots$ & & & $\ldots$ & $\ldots$ & & & $\ldots$ & & & $\ldots$ & $\ldots$ & \\
\hline Gross financing need $2 /$ & 14.7 & 12.6 & 11.1 & & & 12.7 & 13.2 & 12.2 & 11.4 & 10.9 & 10.4 & & 8.4 & 10.6 & \\
\hline $\mathrm{PV}$ of public sector debt-to-revenue and grants ratio (in percent) & 115.9 & 62.6 & 129.1 & & & 124.0 & 140.7 & 150.6 & 155.5 & 156.1 & 152.1 & & 133.2 & 137.6 & \\
\hline $\mathrm{PV}$ of public sector debt-to-revenue ratio (in percent) & 156.2 & 89.8 & 168.9 & & & 173.2 & 183.5 & 192.6 & 195.0 & 194.9 & 187.8 & & 154.2 & 158.3 & \\
\hline $\mathrm{o} / \mathrm{w}$ external 3/ & & & 80.9 & & & 84.5 & 104.6 & 121.6 & 128.7 & 130.7 & 127.5 & & 111.0 & 100.1 & \\
\hline Debt service-to-revenue and grants ratio (in percent) 4/ & 7.4 & 6.2 & 9.0 & & & 7.9 & 7.2 & 7.6 & 8.5 & 10.0 & 10.9 & & 13.8 & 19.8 & \\
\hline Debt service-to-revenue ratio (in percent) $4 /$ & 10.0 & 8.8 & 11.8 & & & 11.0 & 9.4 & 9.7 & 10.7 & 12.4 & 13.4 & & 16.0 & 22.8 & \\
\hline Primary deficit that stabilizes the debt-to-GDP ratio & 31.2 & 3.0 & 4.5 & & & 2.7 & 3.2 & 3.1 & 3.2 & 2.9 & 2.1 & & 1.4 & -0.4 & \\
\hline \multicolumn{16}{|l|}{ Key macroeconomic and fiscal assumptions } \\
\hline Real GDP growth (in percent) & 6.9 & 7.3 & 6.4 & 6.8 & 0.7 & 5.8 & 6.5 & 7.1 & 7.5 & 7.5 & 7.5 & 7.0 & 7.5 & 7.5 & 7.5 \\
\hline Average nominal interest rate on forex debt (in percent) & 0.3 & 1.0 & 0.7 & 0.7 & 0.3 & 0.5 & 0.3 & 1.3 & 1.9 & 2.0 & 2.2 & 1.4 & 3.0 & 4.5 & 3.4 \\
\hline Average real interest rate on domestic debt (in percent) & 7.7 & -5.6 & -2.8 & -0.5 & 5.0 & 6.1 & 4.6 & 4.2 & 3.5 & 4.7 & 6.9 & 5.0 & 10.1 & 13.1 & 11.4 \\
\hline Real exchange rate depreciation (in percent,+ indicates depreciation) & -7.4 & 3.6 & -9.9 & -0.2 & 6.0 & -1.0 & $\ldots$ & & & $\ldots$ & & $\ldots$ & $\ldots$ & $\ldots$ & $\ldots$ \\
\hline Inflation rate (GDP deflator, in percent) & -0.5 & 11.6 & 16.9 & 7.8 & 5.2 & 5.9 & 4.9 & 4.7 & 5.0 & 5.0 & 3.5 & 4.9 & 4.9 & 4.9 & 4.9 \\
\hline Growth of real primary spending (deflated by GDP deflator, in percent) & 0.2 & 0.0 & 0.1 & 0.1 & 0.1 & 0.2 & 0.0 & 0.1 & 0.1 & 0.1 & 0.1 & 0.1 & 0.1 & 0.1 & 0.1 \\
\hline Grant element of new external borrowing (in percent) & $\ldots$ & $\ldots$ & $\ldots$ & $\ldots$ & $\ldots$ & 49.2 & 18.7 & 16.5 & 18.5 & 21.8 & 17.6 & 23.7 & 4.4 & -2.9 & $\ldots$ \\
\hline
\end{tabular}

1/ [Indicate coverage of public sector, e.g., general government or nonfinancial public sector. Also whether net or gross debt is used.]

2/ Gross financing need is defined as the primary deficit plus debt service plus the stock of short-term debt at the end of the last period.

3/ Revenues excluding grants.

4/ Debt service is defined as the sum of interest and amortization of medium and long-term debt.

$5 /$ Historical averages and standard deviations are generally derived over the past 10 years, subject to data availability. 
Table 4.Tanzania: Sensitivity Analysis for Key Indicators of Public Debt 2010-2030

\begin{tabular}{|c|c|c|c|c|c|c|c|c|}
\hline & \multicolumn{8}{|c|}{ Projections } \\
\hline & 2010 & 2011 & 2012 & 2013 & 2014 & 2015 & 2020 & 2030 \\
\hline \multicolumn{9}{|c|}{ PV of Debt-to-GDP Ratio } \\
\hline Baseline & 27 & 29 & 31 & 32 & 33 & 34 & 31 & 34 \\
\hline \multicolumn{9}{|l|}{ A. Alternative scenarios } \\
\hline A1. Real GDP growth and primary balance are at historical averages & 27 & 27 & 26 & 26 & 25 & 25 & 28 & 47 \\
\hline A2. Primary balance is unchanged from 2010 & 27 & 27 & 27 & 27 & 26 & 27 & 31 & 51 \\
\hline A3. Permanently lower GDP growth $1 /$ & 27 & 29 & 32 & 33 & 33 & 34 & 33 & 40 \\
\hline \multicolumn{9}{|l|}{ B. Bound tests } \\
\hline B1. Real GDP growth is at historical average minus one standard deviations in 2011-2012 & 27 & 30 & 32 & 33 & 34 & 35 & 34 & 38 \\
\hline B2. Primary balance is at historical average minus one standard deviations in 2011-2012 & 27 & 28 & 29 & 30 & 30 & 31 & 29 & 33 \\
\hline B3. Combination of B1-B2 using one half standard deviation shocks & 27 & 27 & 28 & 29 & 30 & 30 & 29 & 34 \\
\hline B4. One-time 30 percent real depreciation in 2011 & 27 & 35 & 37 & 37 & 37 & 38 & 37 & 44 \\
\hline \multicolumn{9}{|c|}{ PV of Debt-to-Revenue Ratio 2/ } \\
\hline Baseline & 124 & 141 & 151 & 155 & 156 & 152 & 133 & 138 \\
\hline \multicolumn{9}{|l|}{ A. Alternative scenarios } \\
\hline A1. Real GDP growth and primary balance are at historical averages & 124 & 127 & 126 & 122 & 118 & 113 & 116 & 188 \\
\hline A2. Primary balance is unchanged from 2010 & 124 & 130 & 131 & 129 & 125 & 122 & 131 & 205 \\
\hline A3. Permanently lower GDP growth $1 /$ & 124 & 141 & 151 & 157 & 158 & 155 & 140 & 161 \\
\hline \multicolumn{9}{|l|}{ B. Bound tests } \\
\hline B1. Real GDP growth is at historical average minus one standard deviations in 2011-2012 & 124 & 141 & 154 & 159 & 161 & 158 & 143 & 155 \\
\hline B2. Primary balance is at historical average minus one standard deviations in 2011-2012 & 124 & 134 & 139 & 144 & 145 & 141 & 124 & 132 \\
\hline B3. Combination of B1-B2 using one half standard deviation shocks & 124 & 131 & 133 & 139 & 140 & 138 & 124 & 137 \\
\hline B4. One-time 30 percent real depreciation in 2011 & 124 & 167 & 176 & 178 & 177 & 172 & 156 & 177 \\
\hline B5. 10 percent of GDP increase in other debt-creating flows in 2011 & 124 & 183 & 193 & 197 & 196 & 190 & 164 & 158 \\
\hline
\end{tabular}

Debt Service-to-Revenue Ratio 2/

Baseline

A1. Real GDP growth and primary balance are at historical averages

A2. Primary balance is unchanged from 2010

A3. Permanently lower GDP growth $1 /$

\section{B. Bound tests}

B1. Real GDP growth is at historical average minus one standard deviations in 2011-2012

B2. Primary balance is at historical average minus one standard deviations in 2011-2012

B3. Combination of B1-B2 using one half standard deviation shocks

B4. One-time 30 percent real depreciation in 2011

B5. 10 percent of GDP increase in other debt-creating flows in 2011

$\begin{array}{rrrrrrrr}8 & 7 & 8 & 9 & 10 & 11 & 15 & 22 \\ 8 & 7 & 7 & 7 & 8 & 9 & 13 & 19 \\ 8 & 7 & 7 & 6 & 8 & 9 & 13 & 20 \\ 8 & 8 & 9 & 11 & 13 & 14 & 20 & 32 \\ 8 & 7 & 12 & 15 & 16 & 16 & 18 & 23\end{array}$

Sources: Country authorities; and staff estimates and projections.

1/ Assumes that real GDP growth is at baseline minus one standard deviation divided by the square root of the length of the projection period.

2/ Revenues are defined inclusive of grants. 
Figure 1. Tanzania: Indicators of Public and Publicly Guaranteed External Debt under Alternatives Scenarios, 2010-2030 1/
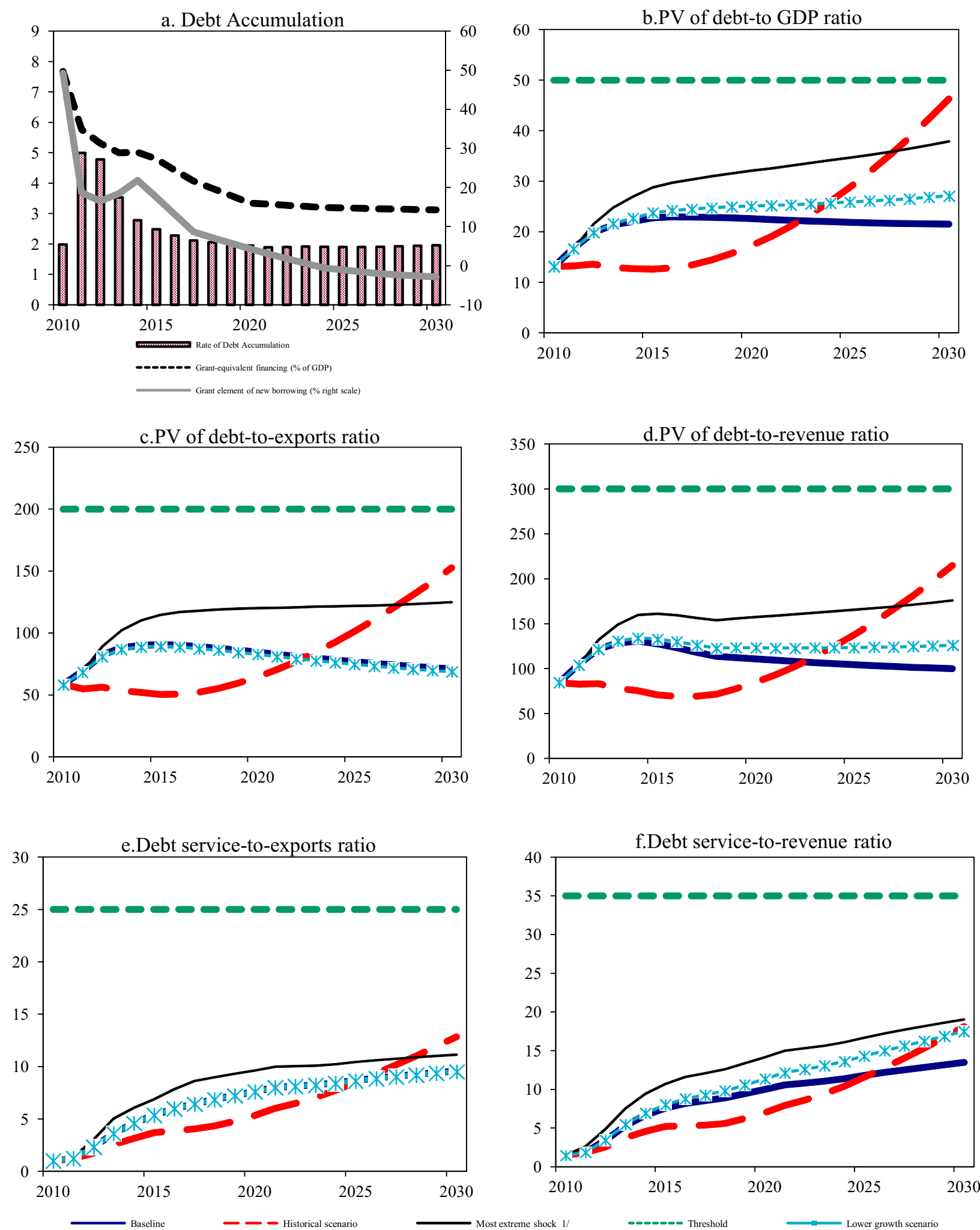

Sources: Country authorities; and staff estimates and projections.

1/ The most extreme stress test is the test that yields the highest ratio in 2020. In figure b. it corresponds to a Terms shock; in c. to a Terms shock; in d. to a Terms shock; in e. to a Exports shock and in figure f. to a One-time depreciation shock

\section{CInternational Monetary Fund. Not for Redistribution}


Figure 2.Tanzania: Indicators of Public Debt Under Alternative Scenarios, 2010-2030 1/
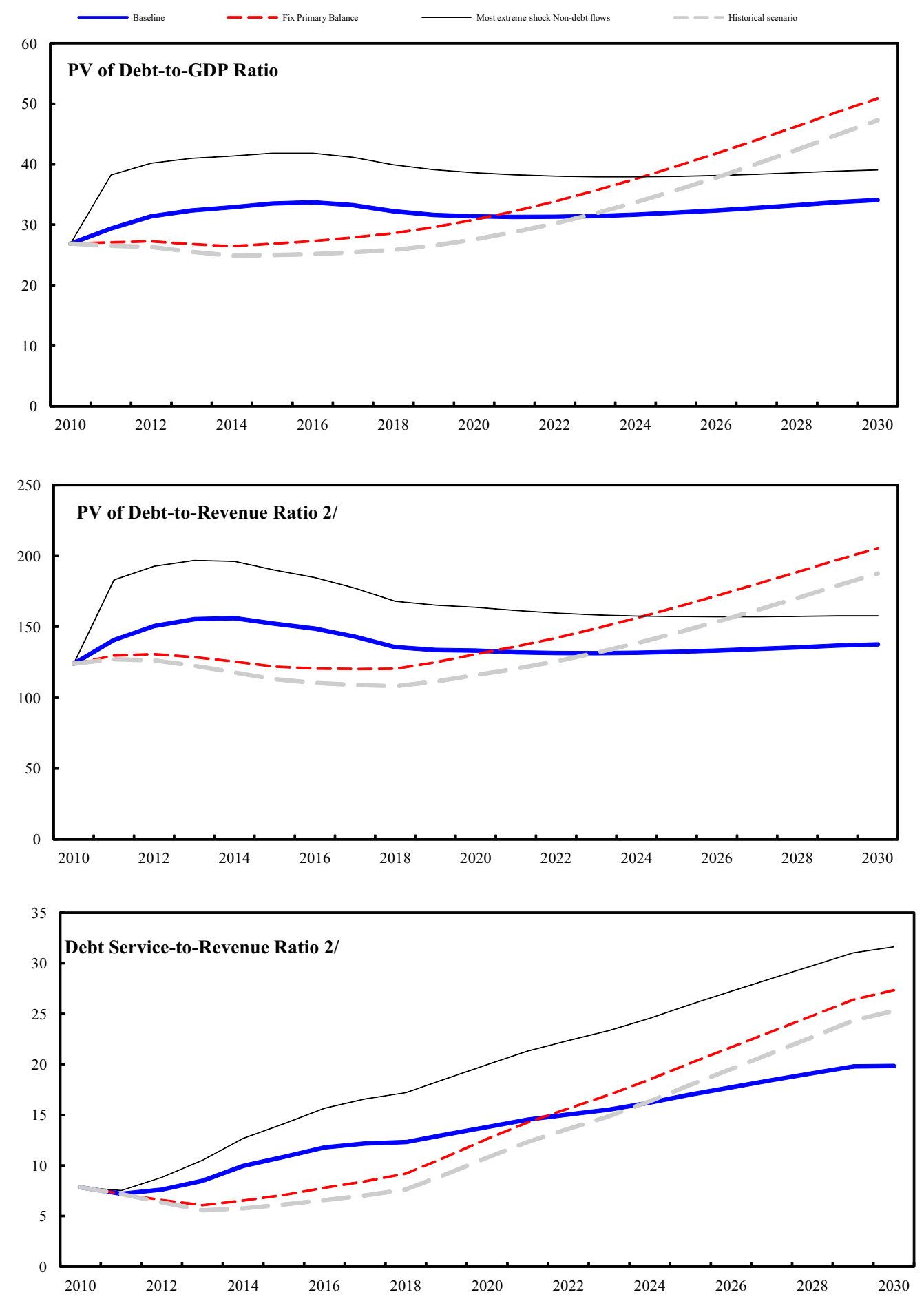

Sources: Country authorities; and staff estimates and projections.

$1 /$ The most extreme stress test is the test that yields the highest ratio in 2020 .

2 / Revenues are defined inclusive of grants. 
INTERNATIONAL MONETARY FUND

UNITED REPUBLIC OF TANZANIA

Seventh Review Under the Policy Support Instrument, Second Review Under the Exogenous Shocks Facility, and Request for a New Three-Year Policy Support Instrument-Informational Annex

Prepared by the African Department

(In consultation with other departments)

May 18, 2010

\footnotetext{
- $\quad$ Relations with the Fund.

- Relations with the World Bank Group.

- Joint Bank-Fund Work Program. Describes Bank-Fund collaboration from May 2010 May 2011.
} 


\section{APPENDIX I. TANZANIA: RELATIONS WITH THE FUND}

(As of March 31, 2010)

I. Membership Status: Joined: September 10, 1962;

$\underline{\text { Article VIII }}$

II. General Resources Account:

SDR Million

\%Quota

Quota

198.90

100.00

Fund holdings of currency

188.90

94.97

Reserve Tranche Position

10.00

5.03

Lending to the Fund

Notes Issuance

Holdings Exchange Rate

\section{SDR Department:}

Net cumulative allocation

Holdings

IV. Outstanding Purchases and Loans:

ESF Arrangements

ECF Arrangements
SDR Million

190.51

158.70

SDR Million

198.90

11.20
\%Allocation

100.00

83.30

\%Quota

100.00

5.63

\section{Latest Financial Arrangements:}

\begin{tabular}{lcccc} 
Type & $\begin{array}{c}\text { Date of } \\
\text { Arrangement }\end{array}$ & $\begin{array}{c}\text { Expiration } \\
\text { Date }\end{array}$ & $\begin{array}{c}\text { Amount Approved } \\
\text { (SDR Million) }\end{array}$ & $\begin{array}{c}\text { Amount Drawn } \\
\text { (SDR Million) }\end{array}$ \\
\cline { 2 - 2 } & May 29,2009 & May 28, 2010 & 218.79 & 198.90 \\
ECF $^{1}$ & Aug 16, 2003 & Feb 26, 2007 & 19.60 & 19.60 \\
ECF $^{1}$ & Apr 04, 2000 & Aug 15, 2003 & 135.00 & 135.00
\end{tabular}

${ }^{1}$ Formerly PRGF.

VI. Projected Payments to Fund ${ }^{2}$

(SDR Million; based on existing use of resources and present holdings of SDRs):

Principal

Charges/Interest

Total

$\begin{array}{llllr}\frac{2}{2010} & \underline{2011} & \underline{2012} & \underline{2013} & \underline{2014} \\ 0.28 & 1.40 & 1.96 & \underline{2.24} & 18.15 \\ \underline{0.09} & \underline{0.09} & \underline{0.61} & \underline{0.60} & \underline{0.60} \\ \underline{0.37} & \underline{1.49} & \underline{2.57} & \underline{2.84} & \underline{18.75}\end{array}$

${ }^{2}$ When a member has overdue financial obligations outstanding for more than three months, the amount of such arrears will be shown in this section. 


\section{Implementation of HIPC Initiative:}

Enhanced

I. Commitment of HIPC assistance

Framework

Decision point date

Apr 2000

Assistance committed

by all creditors (US\$ Million) ${ }^{1}$

$2,026.00$

Of which: IMF assistance (US\$ million)

119.80

(SDR equivalent in millions)

88.95

Completion point date

Nov 2001

II. Disbursement of IMF assistance (SDR Million)

Assistance disbursed to the member

Interim assistance

26.68

Completion point balance

Additional disbursement of interest income ${ }^{2}$

\section{Total disbursements}

96.40

${ }^{1}$ Assistance committed under the original framework is expressed in net present value (NPV) terms at the completion point, and assistance committed under the enhanced framework is expressed in NPV terms at the decision point. Hence these two amounts can not be added.

${ }^{2}$ Under the enhanced framework, an additional disbursement is made at the completion point corresponding to interest income earned on the amount committed at the decision point but not disbursed during the interim period.

\section{Implementation of Multilateral Debt Relief Initiative (MDRI):}

I. DRI-eligible debt (SDR Million) ${ }^{1}$

Financed by: MDRI Trust

Remaining HIPC resources

\section{Debt Relief by Facility (SDR Million)}

\section{$\underline{\text { Eligible Debt }}$}

\section{$\underline{\text { Delivery }}$}

Date

$\underline{\text { GRA }}$

PRGF

$\underline{\text { Total }}$

January 2006

N/A

234.03

\footnotetext{
${ }^{1}$ The MDRI provides 100 percent debt relief to eligible member countries that qualified for the assistance. Grant assistance from the MDRI Trust and HIPC resources provide debt relief to cover the full stock of debt owed to the Fund as of end-2004 that remains outstanding at the time the member qualifies for such debt relief.
} 


\section{Safeguards Assessments:}

Safeguards assessments of the Bank of Tanzania (BoT) were completed in December 2003, August 2008, and November 2009. The 2009 assessment found that, while the BoT had taken steps to strengthen its safeguards framework, risks remained due to delays in implementing certain priority recommendations from the 2008 voluntary assessment. The external audit and financial reporting continued to comply with international standards, but the audited results were published with significant delays. The assessment recommended a bank-wide risk assessment, outlined steps needed to modernize the internal audit function, and proposed measures to enhance transparency of financial, control and compliance matters. In January 2010, the BoT published its audited financial statements for the year ended June 30, 2009. The implementation of other measures is in progress.

\section{Exchange Rate Arrangement:}

The currency of Tanzania is the Tanzania shilling. Under the revised classification methodology, Tanzania has a floating exchange rate arrangement. The official exchange rate is determined in relation to the rate established in the interbank market for foreign exchange. The middle rate in terms of the U.S. dollar, the intervention currency, was T Sh 1,345 per U.S. dollar as of end-March, 2010. The exchange system is free of restrictions on the making of payments and transfers for current international transactions.

\section{Article IV Consultation:}

The most recent Article IV consultation was concluded on June 8, 2009 (Country Report No. 09/179).

\section{Resident Representative:}

Mr. David O. Robinson has been the Senior Resident Representative since March 2007. 


\section{Appendix II. TANZania: Relations With the World Bank Group}

(As of March 31, 2010)

Beginning with a credit from the World Bank's International Development Association (IDA) for education in 1963, over US\$ 7.2 billion in credits and US\$ 274 million in grants have so far been approved. As of March 31, 2010, the IDA's country portfolio comprises of 23 active operations with commitments of US\$2,425.88 million in all major sectors. The undisbursed balance stands at US\$1,347.1 million.

The Country Assistance Strategy (CAS) provides the overall framework of the IDA as well as IFC's activities in Tanzania. The current CAS was prepared as part of the Joint Assistance Strategy for the United Republic of Tanzania (JAST) jointly with 22 development partners (DPs), aligned with the priorities of Tanzania's poverty reduction strategy (PRS), known as MKUKUTA for the Mainland and MKUZA for Zanzibar. While the current CAS was originally designed to cover from FY2007 to FY2010, it is now extended by one year to cover FY2011.

A mix of exogenous and endogenous shocks combined with slower than anticipated reforms has necessitated adjustments to the lending program during the current CAS period.

- Global food and financial crises during 2008-09. The Bank responded promptly to the government's requests to help mitigate the impact of global crises. In the face of price hikes in food and farm inputs, the Accelerated Food Security Program - a US\$220 million package of an Emergency Recovery Credit and Additional Financing for agricultural and social fund projects - was added to the country program in FY09 under the Global Food Crisis Response Program. To contribute to the government's FY09/10 fiscal stimulus package, a supplemental financing of US\$170 million to Poverty Reduction Strategy Credit (PRSC) 7 was approved in mid-FY10. Under the Crisis Response Window established in December 2009, US\$110 million will be provided in FY10 on top of the regular IDA15 allocation.

- Modest progress in key reforms and governance-related issues. Grand corruption cases, modest progress in key reforms and mediocre overall performance against the general budget support (GBS) Performance Assessments Frameworks (PAFs) over the past few years have led to a stagnant or declining level of annual budget support. A series of grand corruption cases that have surfaced since 2008 negatively affected the level and timing of GBS for FY09 provided by 14 DPs, including PRSC6. The PRSC7, projected in the CAS to be US\$300 million, was reduced to US\$190 million. The PRSC8 is likely to face a more significant reduction on performance grounds.

- A decrease in the 2008 Country Policy and Institutional Assessment (CPIA) rating, resulting in a lower-than-anticipated IDA15 allocation to Tanzania for FY09/10. Tanzania's weaker performance led to the decline in Tanzania's 2008 CPIA rating from 3.9 to 3.8, which in turn resulted in a downward adjustment in the expected IDA15 envelope. The projected total IDA15 resources were reduced by about US\$312 million. With an expected frontloading of about 27 percent for FY09-10, the 
uncommitted balance available for FY11 is expected to be only about US\$270 million, forcing changes in the lending program. As a result, a couple of FY11 pipeline operations will have to be scheduled for early FY12, including PRSC9 - the first of a new programmatic series in support of GOT's FY11/12 budget.

- Rapidly growing regional initiatives. Growing regional initiatives and commitment have increased the number and volume of regional projects for Tanzania. The government has shown an increased interest in regional projects, recognizing the potential benefits from, for instance, network effects from infrastructure programs and positive externalities from environmental initiatives. In 2007, there were only six regional projects involving Tanzania with a total commitment of US\$68.9 million specifically for Tanzania. By the end of FY09, this had grown to eleven projects with Tanzanian commitments of US\$216.4 million (equivalent to about 9 percent of the country portfolio). Since two-thirds of regional projects or components are financed through a separate regional IDA envelope, it could have a substantial impact on resource availability for Tanzania.

As a result, investment operations have been scaled up substantially and their sequencing and size have been adjusted to reflect the project readiness and absorptive capacity. Several infrastructure and human development projects have been scaled up from the original plans. Several new projects (e.g., housing finance, regional agricultural productivity, backbone transmission, and two urban infrastructure projects) have been added. The share of PRSCs in the FY07-10 lending program has declined from 50 percent as envisaged in the CAS to 32 percent.

\section{The regional integration agenda is supported largely through projects for trade facilitation, transport, energy, and communication network. The efforts to create a} stronger EAC are supported through the East Africa Trade and Transport Facilitation Project and the Regional Trade Facilitation Project. In addition, several studies have been undertaken in support of Tanzania's efforts to further regional integration. These include among other how to build Tanzania as a regional trade hub, a strategic plan for Export Processing Zones in Tanzania, and a study on non-tariff measures on goods in EAC. A follow up to the latter is planned for FY11 in combination with a study on professional services. Lastly, IFC and Foreign Investment Advisory Services (FIAS) have provided analysis of the regulatory framework for Special Economic Zones in an effort to assist harmonization of regulatory frameworks across the member countries of EAC.

Analytical and advisory (AAA) work. Substantial AAA work has been carried out since 2007, many in close collaboration with other DPs. It includes a series focusing on constraints to economic growth and how growth can contribute to a society with less poverty. An extensive number of short policy notes in specific areas have been produced with the intention to provide key issues, options, and recommendations in a clear but concise and readable manner, for instance in the cashew, charcoal, and coffee sectors. The majority of AAA has been carried out under MKUKUTA Cluster I (growth and reduction of poverty). At the same time AAA has addressed cross-sectoral issues such as national poverty assessments 
and Public Expenditure and Financial Accountability Review (PEFAR). Finally, the Bank's AAA has contributed substantially to addressing recent corruption scandals through strengthening of the Controller Auditor General's office and policy dialogue in connection with triggers for the PRSC series.

International Finance Corporation (IFC). IFC has mobilized over \$185 million in investments for the country's economy to-date and offered a broad range of advisory services to support the private sector. IFC's strategy in Tanzania focuses on supporting micro, small and medium enterprises through financial intermediaries, developing infrastructure by providing long term finance for large projects, and investing in agribusiness, tourism and other key economic sectors. It has adopted an opportunistic approach, looking for investment opportunities across sectors. Its portfolio has significantly increased from US\$35 million in February 2007 to US\$98 million at the end of 2009. IFC has forged partnerships with local banks to foster joint ventures aimed at supporting these projects with local currency financing, as well as developing micro- and SME-finance products. The Credit Reference Bureau program provides support to the BoT for the implementation, licensing, and monitoring of bureaus and the implementation of a credit reporting system. IFC has been exploring private sector participation in utilities and parastatals, and is interested in providing finance for Independent Power Procures projects in the country. However, its US\$44 million loan to the Tanzania Railway Limited to help rehabilitate and develop its railway network has stalled after disbursing a first installment, pending agreement of a new business plan between the GoT and the operator.

Multilateral Investment Guarantee Agency (MIGA). Tanzania became a member of MIGA in 1992. At present, MIGA does not have any exposure in Tanzania and no projects in its pipeline. 


\section{Appendix III: Tanzania- Joint Bank-Fund Work Program MAY 2010-MAY 2011}

\begin{tabular}{|c|c|c|c|}
\hline Title & Products & $\begin{array}{l}\text { Provisional } \\
\text { timing of } \\
\text { missions } \\
\text { (if relevant) }\end{array}$ & $\begin{array}{l}\text { Expected delivery } \\
\text { date }\end{array}$ \\
\hline \multicolumn{4}{|c|}{ A. Mutual information on relevant work programs } \\
\hline \multirow[t]{4}{*}{$\begin{array}{l}\text { Key elements of } \\
\text { World Bank work } \\
\text { program in next } \\
12 \text { months }\end{array}$} & $\begin{array}{l}\text { Poverty Reduction Support Credits } \\
\text { (PRSC-8) to support policy reforms to } \\
\text { enhance productivity and } \\
\text { competitiveness, improve effectiveness } \\
\text { of service delivery, public sector } \\
\text { management including PFM, and } \\
\text { human development issues through } \\
\text { budget support. Implementation } \\
\text { completion and result (ICR) report to be } \\
\text { prepared for PRSC4-8 series after } \\
\text { PRSC-8 delivery. }\end{array}$ & & June 2010 \\
\hline & $\begin{array}{l}\text { The work program will continue to } \\
\text { concentrate on areas within the existing } \\
\text { and new lending portfolio: primary, } \\
\text { secondary, and higher education; health } \\
\text { sector development; water and } \\
\text { sanitation; social action fund; } \\
\text { agriculture; natural resource } \\
\text { management including minerals ; } \\
\text { environment; road and railway } \\
\text { infrastructure; power sector } \\
\text { restructuring; housing finance; } \\
\text { statistical capacity; urban; fiscal } \\
\text { decentralization; private sector } \\
\text { competitiveness; and public sector } \\
\text { reform. Increasing regional portfolio } \\
\text { in agriculture, health, power, and } \\
\text { environment. }\end{array}$ & & Continuous \\
\hline & $\begin{array}{l}\text { Public expenditure review with the } \\
\text { focus on rapid budget analysis (RBA) } \\
\text { and Public Expenditure and Financial } \\
\text { Accountability Report (PEFAR). }\end{array}$ & & $\begin{array}{l}\text { November } 2010 \\
\text { (RBA); May } 2011 \\
\text { (PEFAR) }\end{array}$ \\
\hline & Poverty Assessment In-depth Analysis & & September 2010 \\
\hline
\end{tabular}




\begin{tabular}{|l|l|l|l|}
\hline $\begin{array}{l}\text { IMF work } \\
\text { program in next } \\
12 \text { months }\end{array}$ & $\begin{array}{l}\text { Seventh PSI Review, second ESF } \\
\text { Review, and New PSI }\end{array}$ & March 2010 & May 2010 \\
\cline { 2 - 4 } & First PSI Review & September 2010 & November 2010 \\
\cline { 2 - 4 } & $\begin{array}{l}\text { Second PSI Review and Article IV } \\
\text { Consultation }\end{array}$ & March 2011 & May 2011 \\
\hline
\end{tabular}

\begin{tabular}{|c|c|c|c|}
\hline \multicolumn{4}{|c|}{ B. Requests for work program inputs } \\
\hline \multirow[t]{3}{*}{$\begin{array}{l}\text { Fund request to } \\
\text { Bank }\end{array}$} & $\begin{array}{l}\text { 1. Assessment of key infrastructure projects } \\
\text { and sectoral programs. }\end{array}$ & & Continuous \\
\hline & 2. Assessment of social security funds & & Continuous \\
\hline & $\begin{array}{l}\text { 3. Inputs on the design of a social } \\
\text { protection framework }\end{array}$ & & Continuous \\
\hline \multirow[t]{4}{*}{$\begin{array}{l}\text { Bank requests to } \\
\text { Fund }\end{array}$} & $\begin{array}{l}\text { 1. Monitoring of government contracting of } \\
\text { non-concessional borrowing. }\end{array}$ & & Continuous \\
\hline & $\begin{array}{l}\text { 2. Monitoring of steps to strengthen } \\
\text { corporate governance of the BoT. }\end{array}$ & & Continuous \\
\hline & 3. Sharing macro-framework updates & & Continuous \\
\hline & $\begin{array}{l}\text { 4. Statement of fiscal risk and contingent } \\
\text { liabilities }\end{array}$ & & Continuous \\
\hline \multicolumn{4}{|c|}{ C. Agreement on joint products and missions } \\
\hline \multirow{4}{*}{$\begin{array}{l}\text { Joint products in } \\
\text { next } 12 \text { months }\end{array}$} & 1. Collaborate on the Joint DSA. & March 2010 & May 2010 \\
\hline & $\begin{array}{l}\text { 2. Collaborate on JSAN following approval } \\
\text { of the new Poverty Reduction Strategy. }\end{array}$ & September 2010 & November 2010 \\
\hline & $\begin{array}{l}\text { 3. Collaborate together with other } \\
\text { development partners on the annual review } \\
\text { of general budget support. }\end{array}$ & Early 2011 & Early 2011 \\
\hline & $\begin{array}{l}\text { 4. Collaborate on PFM reform program } \\
\text { in the context of multi-donor support of the } \\
\text { Public Financial Management Reform } \\
\text { Program. }\end{array}$ & & Continuous \\
\hline
\end{tabular}


Press Release No. 10/227

International Monetary Fund

FOR IMMEDIATE RELEASE

Washington, D.C. 20431 USA

June 4, 2010

\section{IMF Executive Board Completes Seventh Review under Tanzania's PSI; Approves a Three-Year PSI, Completes Second Review Under the ESF and Approves US\$29.1 Million Disbursement}

The Executive Board of the International Monetary Fund (IMF) completed today the seventh review of Tanzania's economic performance under the Policy Support Instrument (PSI) and approved a new three-year Instrument ${ }^{1}$. The previous PSI was approved by the Executive Board in 2007 (see Press Release No. 07/26) and has been extended until June 4, 2010.

The Board also completed the second review of Tanzania's performance under an arrangement under the Exogenous Shocks Facility (ESF). The completion of the review will enable the third and final disbursement of SDR 19.89 million (US\$29.1 million). The 12month, SDR 218.79 million (US\$320.6 million) high access ESF arrangement was approved on May 29, 2009 (see Press Release No. 09/190). On May 24, 2010, the Board extended the period of the one-year arrangement to June 18,2010, to permit completion of the second review.

The new PSI for Tanzania aims at maintaining macroeconomic stability and supporting accelerated growth, creating additional fiscal space, including to finance infrastructure investment, while enhancing the return on public spending. It will also support the new poverty reduction and growth strategy (MKUKUTA II) covering 2010-15 that is expected to be approved in June.

The IMF's framework for PSIs is designed for low-income countries that may not need, or want, IMF financial assistance, but still seek IMF advice, monitoring, and endorsement of their policies (see Public Information Notice No. 05/145).

Following the Executive Board's discussion on Tanzania, Mr. Murilo Portugal, Deputy Managing Director and Acting Chair, stated:

1 "Discussions were held in Dar es Salaam during March 2-16 and concluded in Washington during April 19-26 and reflect information available at that time."

Washington, D.C. 20431 • Telephone 202-623-7100 • Fax 202-623-6772 • www.imf.org 
"The Tanzanian economy is emerging from a slowdown associated with the global crisis and natural disasters. The authorities' macroeconomic policy response, including temporary and targeted fiscal stimulus measures and supportive monetary policy, helped mitigate the downturn. Inflation remained high throughout 2009, but has since abated to single digits. A stronger-than-envisaged current account and frontloaded donor assistance enabled maintenance of a comfortable reserve position. Progress under Tanzania's poverty reduction and growth strategy is to be commended, with roughly half the Millennium Development Goals expected to be achieved by 2015 . Continued prudent macroeconomic policies, progress on structural reforms, and improvements in the business climate will be important to sustain these achievements.

"The near-term policy challenge is to support the economic recovery, while gradually withdrawing the fiscal stimulus as growth strengthens. The central bank will need to monitor carefully the liquidity conditions to guard against the emergence of inflation or exchange rate pressures.

"The new three-year Policy Support Instrument will support implementation of the authorities' new poverty reduction and growth strategy (MKUKUTA II), to be finalized shortly. The strategy reorients government spending toward infrastructure investment, while preserving priority social spending, in order to accelerate progress in reducing poverty.

"The authorities aim to create needed fiscal space through rationalizing nonpriority spending, further enhancing revenue collection, limited nonconcessional borrowing, and greater use of public-private partnerships. Debt sustainability analysis points to a low risk from this strategy, and reforms are underway to strengthen debt management, enhance strategic planning, and improve the business environment.

"The banking system is well capitalized. The authorities have developed an action plan to implement the recommendations of the recent FSAP. Although the global crisis had a limited impact on banks, measures to contain vulnerabilities and strengthen the crisis management framework have been undertaken," Mr. Portugal added. 


\section{ANNEX}

\section{Recent Economic Developments}

The Tanzanian economy is starting to emerge from a slowdown associated with the global economic crisis. Prudent fiscal policy in recent years provided Tanzania with sufficient fiscal space to implement a countercyclical fiscal response to the crisis that helped mitigate weaker external demand and a sharp slowdown in credit growth. Inflation rose into double digits during 2008-09, driven by supply-side shocks that pushed up food prices, but fell sharply in the first half of 2010 as these effects subsided. Growth is projected to reach 6.2 percent in 2010 from 5.5 percent in 2009, while monetary and fiscal stimulus will be withdrawn gradually in order to protect the still nascent recovery.

The medium-term outlook remains favorable. Looking forward, the fiscal deficit will remain higher than in the recent past to accommodate higher infrastructure spending. Spending will be reoriented to growth-enhancing projects, while preserving priority social spending. Monetary policy will seek to support stronger growth while maintaining low inflation without excessive volatility in the exchange rate. Growth is expected to accelerate to 7.5 percent by 2012/13, while inflation would stabilize at around 5 percent. Uncertainties for the growth outlook include the rate of return on public investment and the responsiveness of private sector activity, while the inflation path will remain vulnerable to external shocksespecially related to food supply and energy prices.

\section{Program Summary}

Tanzania's PSI will be guided by the objectives and policies outlined in the new Poverty Reduction Strategy to be approved in June 2010. Fiscal policy will seek to balance a gradual reining in of the stimulus while reorienting spending in line with the poverty-reduction objectives. For 2010/11, the deficit before grants will be reduced to 10.8 percent of GDP from 12 percent currently projected for 2009/10. Monetary policy will continue to provide a supportive environment for recovery, while carefully monitoring inflationary pressures to ensure that the reserve money target is consistent with emerging demand. The Bank of Tanzania also plans to maintain regular foreign exchange sales to meet systemic liquidity management objectives. The authorities are updating the CPI basket, and working with East Africa Community counterparts to develop a common core inflation index, which would serve as a better guide to the monetary policy stance. 
Tanzania: Selected Economic and Financial Indicators, 2007/08-2012/13

\begin{tabular}{|c|c|c|c|c|c|c|c|}
\hline \multirow{2}{*}{ - } & \multirow[t]{2}{*}{$2007 / 08$} & \multirow[t]{2}{*}{$2008 / 09$} & \multicolumn{2}{|c|}{$2009 / 10$} & \multirow{2}{*}{$\begin{array}{r}2010 / 11 \\
\text { Proj. }\end{array}$} & \multirow{2}{*}{$\begin{array}{r}2011 / 12 \\
\text { Proj. }\end{array}$} & \multirow{2}{*}{$\begin{array}{r}2012 / 13 \\
\text { Proj. }\end{array}$} \\
\hline & & & Prog. ${ }^{5}$ & Proj. & & & \\
\hline & \multicolumn{7}{|c|}{ (Annual percentage change, unless otherwise indicated) } \\
\hline \multicolumn{8}{|l|}{ National income and prices } \\
\hline Real GDP growth (calendar year) ${ }^{1}$ & 7.1 & 7.4 & 5.0 & 5.5 & 6.2 & 6.7 & 7.5 \\
\hline Real GDP growth (fiscal year) & 7.3 & 6.4 & 5.5 & 5.8 & 6.5 & 7.1 & 7.5 \\
\hline Consumer prices (period average) & 8.4 & 11.8 & $8.0-9.0$ & 10.6 & 5.8 & 5.0 & 5.0 \\
\hline Consumer prices (end of period) & 9.3 & 10.7 & $6.0-8.0$ & 8.0 & 5.0 & 5.0 & 5.0 \\
\hline \multicolumn{8}{|l|}{ External sector } \\
\hline Export, f.o.b (in millions of U.S. dollars) & 2,609 & 2,969 & 3,154 & 3,216 & 3,826 & 4,168 & 4,565 \\
\hline Imports, f.o.b. (in millions of U.S. dollars) & $-5,667$ & $-5,970$ & $-6,070$ & $-6,149$ & $-6,996$ & $-7,539$ & $-8,045$ \\
\hline Export volume & 6.2 & 5.3 & 6.2 & 6.6 & 10.3 & 9.8 & 8.4 \\
\hline Import volume & 13.3 & -1.5 & 8.9 & 6.0 & 6.4 & 5.6 & 4.3 \\
\hline Terms of trade & -7.7 & 10.9 & 7.7 & 8.2 & -2.6 & -2.7 & -1.2 \\
\hline $\begin{array}{l}\text { Nominal effective exchange rate (end of period; } \\
\text { depreciation -) }\end{array}$ & 1.8 & -3.4 & $\cdots$ & $\cdots$ & $\cdots$ & $\cdots$ & $\cdots$ \\
\hline $\begin{array}{l}\text { Real effective exchange rate (end of period; } \\
\text { depreciation -) }\end{array}$ & 2.9 & 6.4 & $\cdots$ & $\cdots$ & $\cdots$ & $\cdots$ & $\cdots$ \\
\hline \multicolumn{8}{|l|}{ Money and credit ${ }^{2}$} \\
\hline Broad money (M3) & 18.1 & 19.0 & 21.1 & 21.6 & 21.0 & $\ldots$ & $\ldots$ \\
\hline Net foreign assets & 4.7 & 15.7 & -5.2 & 13.1 & 13.4 & $\ldots$ & $\ldots$ \\
\hline Net domestic assets & 39.8 & 23.0 & 51.2 & 31.2 & 28.5 & $\ldots$ & $\ldots$ \\
\hline Credit to nongovernment sector & 32.9 & 33.6 & 33.6 & 14.1 & 21.3 & $\ldots$ & $\ldots$ \\
\hline Velocity of money (GDP/M3; average) & 3.7 & 3.7 & 3.5 & 3.3 & 3.3 & $\ldots$ & $\ldots$ \\
\hline \multirow[t]{2}{*}{ Treasury bill interest rate (in percent; end of period) ${ }^{3}$} & 7.8 & 7.0 & $\ldots$ & 4.5 & $\ldots$ & $\ldots$ & $\ldots$ \\
\hline & \multicolumn{7}{|c|}{ (Percent of GDP) } \\
\hline \multicolumn{8}{|l|}{ Public Finance } \\
\hline Revenue (excluding grants) & 15.9 & 15.8 & 16.5 & 15.8 & 16.3 & 16.6 & 16.9 \\
\hline Total grants & 6.9 & 4.9 & 6.2 & 6.3 & 4.9 & 4.6 & 4.3 \\
\hline Expenditure & 22.8 & 25.5 & 27.6 & 27.7 & 27.1 & 26.5 & 25.8 \\
\hline Overall balance (excluding grants) & -6.9 & -9.6 & -11.1 & -12.0 & -10.8 & -10.0 & -8.9 \\
\hline Domestic financing & -1.5 & 0.8 & 1.6 & 1.6 & 1.0 & 1.0 & 1.0 \\
\hline Stock of domestic debt (end of period) & 13.4 & 12.0 & 12.0 & 11.9 & 11.7 & 11.4 & 11.1 \\
\hline \multicolumn{8}{|l|}{ Savings and investment ${ }^{1}$} \\
\hline Resource gap & -16.9 & -13.7 & -12.2 & -12.1 & -11.3 & -11.2 & -10.7 \\
\hline Investment & 29.6 & 29.8 & 28.4 & 27.8 & 28.0 & 28.6 & 28.9 \\
\hline Government & 9.7 & 10.0 & 10.4 & 10.3 & 10.6 & 10.3 & 9.5 \\
\hline Nongovernment ${ }^{4}$ & 20.0 & 19.8 & 18.0 & 17.5 & 17.4 & 18.4 & 19.5 \\
\hline Gross domestic savings & 12.8 & 16.2 & 16.2 & 15.7 & 16.7 & 17.4 & 18.3 \\
\hline \multicolumn{8}{|l|}{ External sector } \\
\hline Current account balance (excluding current transfers) & -14.1 & -13.1 & -11.6 & -11.5 & -11.7 & -11.7 & -11.1 \\
\hline \multirow[t]{2}{*}{ Current account balance (including current transfers) } & -10.6 & -9.8 & -8.6 & -8.3 & -9.3 & -9.4 & -9.0 \\
\hline & \multicolumn{7}{|c|}{ (Millions of U.S. dollars, unless otherwise indicated) } \\
\hline \multicolumn{8}{|l|}{ Balance of payments } \\
\hline $\begin{array}{l}\text { Current account balance (excluding current transfers; } \\
\text { deficit -) }\end{array}$ & $-2,675$ & $-2,804$ & $-2,760$ & $-2,695$ & $-2,994$ & $-3,240$ & $-3,384$ \\
\hline Gross official reserves & 2,660 & 2,925 & 3,277 & 3,461 & 3,755 & 4,085 & 4,365 \\
\hline $\begin{array}{l}\text { In months of imports of goods and nonfactor } \\
\text { services (current year) }\end{array}$ & 4.5 & 4.6 & 5.1 & 5.2 & 5.0 & 5.0 & 5.0 \\
\hline External debt (percent of GDP) & 24.9 & 25.6 & 25.3 & 26.6 & 29.5 & 32.0 & 33.6 \\
\hline
\end{tabular}

Sources: Tanzanian authorities; and IMF staff estimates and projections.

${ }^{1}$ Data are on calendar year basis. For example, 2007/08 data are for calendar year 2007.

${ }^{2}$ Figures for 2008/09 onward reflect a new series based on the Fund's 2000 Monetary and Financial Statistics Manual.

${ }^{3}$ End-year (June) monthly weighted-average yield of 35-, 91-, 182-, and 364-day treasury bills. For 2009/10, figure is for March 2010.

${ }^{4}$ Including change in stocks.

${ }^{5}$ From the sixth review under the PSI, based on previous GDP projection. 


\section{Statement by Mr. Itam, Executive Director United Republic of Tanzania June 4, 2010}

1. My Tanzanian authorities' prudent macroeconomic policies, deepened structural reforms, and swift response to the challenges of the global economic downturn, enabled Tanzania to weather the adverse effects of the crisis. They remain confident that persevering with this macroeconomic framework, they stand a good chance of mitigating the fallout from the sovereign debt risks in advanced economies and the resurging global fuel prices.

2. My authorities are appreciative of the Fund's constructive engagement and support under the PSI and the ESF. Going forward, they are determined to further strengthen their macroeconomic framework, and achieve key national and regional objectives set out in their draft PRSP — Mkukuta II — and East African Community protocols. These will guide the policy thrust of the new PSI.

3. It is against this backdrop, that they are requesting a new three-year arrangement under the PSI to be aligned with the Mkukuta-II objectives and annual budget cycles. In view of the strong performance under the program — with all end-December 2009 quantitative performance/assessment criteria observed with good margins, and implementation of structural reforms on track - they request Directors' support for the completion of the seventh review of the PSI and the second review of the ESF.

\section{Recent economic developments}

4. Tanzania's impressive economic performance, that had been uninterrupted over the past decade reflective of the continuous economic reforms, came under threat from the effects of the global economic downturn. Real GDP growth declined from 7.5 percent in 2008 to 5.5 percent in 2009 as a result of a severe external shock on Tanzania's exports. Deterioration in the external environment has since subsided especially aided by the high prices of gold, an increase in the volume of most of the country's traditional exports, and tourism. International reserves have since rebounded to 5.2 months of import cover.

5. The fiscal outturn in $2009 / 10$ is symptomatic of the challenges the country is facing. Revenue collection continued to stagnate across the board, on account of a combination of a reduction of taxable output, intermittent power supply outages and delayed implementation of some tax measures. Nonetheless, the authorities remained focused on broadening the tax base, implementing structural reforms, and strengthening customs and tax administration. Frontloaded disbursements of budget support by Tanzania's development partners and modest adjustment of non-priority spending will enable the authorities to meet their fiscal targets for the current fiscal year-2009/2010 — albeit with a small shortfall.

6. The thrust of government spending remained in conformity with the overall macroeconomic framework - to mitigate the impact of the global economic downturn in addition 
to maintaining the level of spending on the key MDG clusters and infrastructure. The authorities' modest fiscal stimulus - totaling about 2 percent of GDP — was targeted at protecting employment and incomes, ensuring food security, and protecting investment in infrastructure and key social sectors. The thrust of spending was, however, briefly disrupted by the widespread floods that damaged farming systems and infrastructure. Development expenditure has benefitted from accelerated disbursement of funds for foreign-funded projects, and is expected to be on target by the end of the fiscal year.

7. My authorities' monetary policy continued to support the overall macroeconomic framework with a low interest rate environment. Nonetheless, the cautious stance of the banks in tandem with the global financial turmoil, led to a sharp decline in lending to the private sector, paralleled by an increase in banks' holding of treasury bills. The Bank of Tanzania (BoT) strengthened the operation of its policy instruments for sterilization of liquidity and broadened its monetary policy transparency. As a result, reserve money growth remained on track, and inflation, which had spiked in 2008 and 2009 due to the surge in global food and fuel prices and regional food supply deficits, subsided considerably in line with global price developments and improved regional food supply. However, the non-food price inflation edged up recently as a result of the resurging global fuel prices.

8. The financial system has remained resilient to the global financial crisis, due to its limited exposure and well supervised and capitalized banks. However, the exposure of the banking system to trade financing (crops, horticulture, and other products) and concentration of loan portfolio continue to pose risks from the effects of the global economic downturn. The high proportion of foreign owned banks in the banking system also poses additional risks in terms of the potential for curtailing operations by these banks as economic uncertainties and the global financial crisis become protracted.

\section{Policy framework under the new PSI}

\section{Fiscal policy}

9. My authorities will continue implementing their modest fiscal stimulus - that has a builtin sunset clause - to spur economic growth to its potential and to address the potential fallouts of sovereign debt risks in advanced countries and resurgence of fuel prices. To achieve these objectives, measures including sustaining budgetary spending levels for the 2009/10 fiscal year, scaling-up investment in infrastructure and primary sectors to shore-up economic growth, and deepening structural reforms to improve the business environment will be prioritized.

10. To maintain spending in these priority areas, the authorities will continue to strengthen domestic and concessional resource mobilization in the medium term. Cognizant of the overall resource limitations, they intend to facilitate greater participation of the private sector through PPPs and mobilize a modest level of non-concessional financing-about \$1.5bn over 3 yearswhile remaining vigilant of the contingent liabilities and debt sustainability implications. To that end, they are building capacities in these areas. On the expenditure side, the authorities will take steps to strengthen budget execution, and improve the alignment between policy objectives and 
the budget through prioritization of public investment in line with their Medium-term Public Investment Plan (MPIP).

\section{Monetary and exchange rate policies}

11. My authorities' monetary policy will continue supporting their macroeconomic framework through maintaining sufficient liquidity in the financial system, and low and stable inflation. They will remain vigilant of the inflation pressures arising from the resurgence of global fuel prices. To achieve the objective of anchoring its monetary policy on low and stable inflation, the BoT will further bolster its open market operations, maintain a flexible exchange rate policy, rely substantially on foreign exchange sales for sterilization of liquidity, and continue to improve liquidity forecasting. The BoT is committed to maintain the transparency of its policy stance and promote orderly financial markets. The authorities will also seek to accumulate a level of international reserves consistent with the East African Monetary Union convergence criteria over the medium term.

\section{Financial sector}

12. The BoT will continue to strengthen its supervisory and regulatory services to the financial institutions to stem further exposure to concentration of loan portfolio. It will also strengthen the regulatory framework for financial institutions in line with the recommendations of the Financial System Stability Assessment-Update and the agreed action plan. The BoT will also finalize plans to liberalize capital flows taking into account proper sequencing and capacity to implement supporting policies. Progress in bringing the Social Security Regulatory Authority of the pension sector into operation is also envisaged in the near term.

\section{Structural reforms}

13. My Tanzanian authorities' commitment to the implementation of a wide range of reforms underpins the Mkukuta II objectives. Improving the stock and quality of infrastructure-from road and rail networks to energy and telecommunications and regional infrastructure projects - is a priority with the aim of enhancing productivity in the primary sectors and competitiveness of the country as a whole, and strengthening regional markets.

14. In light of the widening public investments, especially in infrastructure and key primary sectors, the authorities will further strengthen their PFM and public accountability. The capacities of procurement units in all ministries and the public debt management units in all institutions will continue to be strengthened to deal adequately with the evolving environment. The authorities are also determined to continue improving the overall investment climate to further facilitate private sector development, attract and retain FDI, and boost domestic entrepreneurship. In addition to prudent macroeconomic and financial policies, the implementation of the reform program will be deepened.

15. On the regional front, the authorities are committed to deepen the EAC regional integration framework. To that end, they are, together with their partners in the EAC, advancing 
the implementation of the Customs Union and Common Market protocols. They are committed to make progress in the harmonization of monetary and fiscal policies, and the ultimate EAC monetary union. The authorities are especially aware that capital account restrictions have, thus far, sheltered the economy from the inherent shocks, but remain committed to take advantage of the regional experiences while mindful of their capacity to implement supportive policies.

\section{Conclusion}

16. The authorities' macroeconomic fundamentals and continued commitment to strong policies helped the country weather the storm of the global economic crisis better than earlier envisaged. They are aware of the remaining risks and have, therefore, maintained a mix of modest fiscal stimulus and monetary policy easing, as well as deepening reforms to consolidate the economic recovery. In the near term, the authorities are determined to ensure that the program focuses on sustaining macroeconomic stability, enhancing domestic resource mobilization, promoting broad-based and pro-poor growth, and increasing investment in infrastructure, primary sectors and core MDG clusters.

17. In line with the Mkukuta-II objectives and Tanzania Vision 2025, the authorities are determined to ensure that the economy returns to its growth trajectory in the near- to mediumterm. Going forward, continued engagement with the Fund and the support of development partners will enable them achieve their development goals, including poverty reduction and the MDGs. 